\title{
Numerical Fracture Mechanics Analysis of Cracked Fibre- Metal Laminates with Cut-Outs
}

\author{
B. E. Cudzilo and C.L. Tan \\ Department of Mechanical \& Aerospace Engineering \\ Carleton University, Ottawa, Canada, K1S 5B6
}

\begin{abstract}
The boundary element method (BEM) for two-dimensional numerical stress analysis is employed to investigate crack-face bridging of cracked fibre-metal laminates (FML) with cut-outs in this study. The fracture mechanics prediction of crack growth in these perforated laminates involves the interaction of the geometry and crack size, the delamination between the pre-peg and metal layers, and the extent of fibre-bridging of the crack flanks with the stress field caused by the cut-out. The present work investigates the effects of a stress concentration on the fibre-bridging stress and the stress intensity factor of a bridged crack in fibre-metal laminates. A number of cracked configurations are analyzed and the FML, ARALL2, is considered. The bridging stresses on the crack flanks are modeled in the 2-D analysis using power-law expressions and with the mechanical properties of the laminate homogenized through the thickness. An iterative scheme is employed to solve for the bridging stresses as they are not known a priori. Three dimensional finite element method (FEM) analyses are also carried out to confirm the validity of the 2-D BEM models.

FML's with circular cut-outs will contain high bridging stresses near the cutout resulting in fibre failure there, causing a reduction of the extent of fibre bridging of the crack. Results of the study show a likelihood of fibre failure near the edge of the cut-out and this could lead to a reduction of the bridging length. Comparison of the BEM with the FEM stress intensity factors for the range of problems analyzed reveals that the percentage difference is generally less than about $6 \%$, except for a few cases when the power-law index of 0.5 is assumed. The BEM results indicate an increasing bridging stress and stress intensity factor with decreasing bridging length and the benefits of the fibre bridging of the crack are clearly demonstrated. This numerical study confirms that the 2-D BEM models employed can indeed be used to provide a quick and reasonable estimate of the stress intensity factor for a bridged crack in a FML with a circular cut-out.
\end{abstract}




\subsection{INTRODUCTION}

One extrinsic toughening mechanism which reduces the extent of crack opening in fibre-reinforced materials is crack-face fibre-bridging. This mechanism slows crack growth and may even lead to crack arrest. The effect of the crack-tip singular behaviour and its interaction with a hole in a laminate, on the fibre-bridging mechanism, is investigated in this study.

A new class of lightweight, fatigue resistant materials called fibre-metal laminates (FML), developed by the Delft University of Technology in Holland, combine high strength and fatigue properties of composites with good machinability and impact resistance of metals, see, e.g. Marissen $(1988,1989,1991,1994)$ and Guo and Wu (1999). The commercially available ARALL (Aramid Reinforced Aluminum Laminate), GLARE (Glass Reinforced Aluminum Laminate), and CARALL (Carbon Reinforced Aluminum Laminate) consist of alternating layers of thin aluminum alloy sheets bonded by a structural adhesive impregnated by high strength fibres. The use of these fatigue-critical materials in lower wing skins, fuselage, exterior doors, and horizontal and vertical stabilizers can result in weight savings of up to $30 \%$, see, e.g. Marissen (1988), compared to monolithic aluminum.

Much of the experimental and analytical works on fibre-metal laminates reported in the literature consist mainly of fatigue crack propagation studies of plane sheets with no cut-outs. Marissen (1988) has analytically quantified the relationship between bridging stress and delamination zone size with the assumption of a uniform bridging stress profile. Cain and Tan (1997) have analyzed statically loaded crack problems with a range of bridging profiles and showed that the uniform stress profile assumption can only be applied to a central crack geometry. They used a twodimensional simplified boundary element method (BEM) analysis to model a truly three-dimensional problem and showed that it can indeed be used to provide quick estimates of the stress intensity factors.

The present study is expanded from the work of Cain and Tan (1997) to analyze cracks emanating from notches in several geometries with the variation of the fatigue crack size, delamination shape, and the bridging stress profile. To fully understand the bridging effects of FMLs, a three-dimensional study is necessary. This is intractable analytically and is expensive and time consuming numerically when repeated analyses are required. As in Cain and Tan (1997), a two-dimensional BEM study using power-law expressions for the bridging stress distribution with several simplifying assumptions is presented here as a suitable alternative to obtain quick estimates for the stress intensity factors. More specifically, the results obtained from the two-dimensional boundary element method are compared with those obtained from

the three-dimensional finite element method (FEM), and a discussion of the effects of the various parameters on the bridging stress and stress intensity factor is presented. Finally, the effects of reducing the bridging zone length on the bridging stress and stress intensity factor are also presented.

A partially bridged ARALL fibre-metal laminate is analyzed. In the partial bridging cases studied, the bridging zone length is reduced to $75 \%$ and $50 \%$ of the crack length following the assumption that the fibres near the hole have failed. Again, numerical results for partial bridging from the two-dimensional boundary element 
method analysis are presented and compared with those obtained by the threedimensional finite element method. A comparison is made between the case of full bridging, partial bridging, and no bridging of the crack, and the benefit of fibre-bridging in fibre-metal laminates is clearly shown.

\subsection{Analysis Model for Fibre-Bridging}

Fiber-metal laminates, such as ARALL, are hybrid composites that have fracture characteristics similar to conventional composites but with differences in the fracture process. Figure 2.1 shows a 2/1 lay-up (2 layers of metal and one layer of fibre lamina) of a fibre-metal laminate. The crack in fiber-metal laminates initiates and propagates in the metal layers while the composite lamina remains intact. With greater loads, the matrix in the composite layer will begin to crack and eventually the fibers will start breaking. The fibers and matrix transfer the load from the cracked metal layer and thus bridge the crack by restraining further crack opening. There is also load transfer from the metal layer to the fiber layer through a shear stress in the adhesive between the metal and fiber layers. This causes fatigue deformation of the adhesive and results in delamination between the metal and fiber layers. Therefore, there are two inter-related damage systems in such laminates as ARALL, namely, crack growth in the metal layers accompanied by delamination between the fiber and metal layers resulting from adhesive shear deformation.

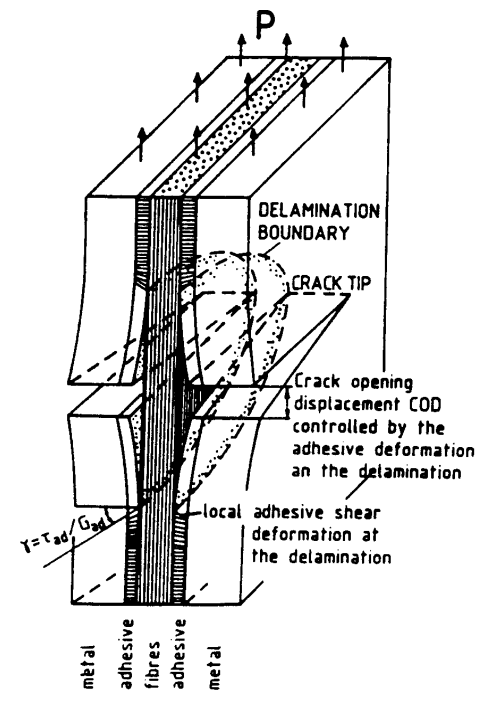

Figure 2.1: 2/1 Lay-up of a Fiber-Metal Laminate, Marissen (1988). 
The function of the adhesive is to impregnate the fibres and bond them to the metal layers. When the laminate is loaded, the adhesive is deformed (see Figure 2.2) due to a local shear stress system. There is a load transfer from the crack bridging fibres to the metal sheets by the shear stresses in the adhesive layer and the resulting shear deformation allows some crack opening and fatigue crack growth (see Figure 2.3). Under fatigue loading, the cyclic load transfer causes cyclic adhesive shear stresses to initiate fatigue delamination of the adhesive between the fibres and the metal sheets. The effect of the adhesive shear on the bridging stresses has been investigated in recent work by Guo and Wu (1999), Guo and Wu (1998), and Takamatsu, et al. (1999). This adhesive deformation is a necessary component for the bridging mechanism in fibremetal laminates.

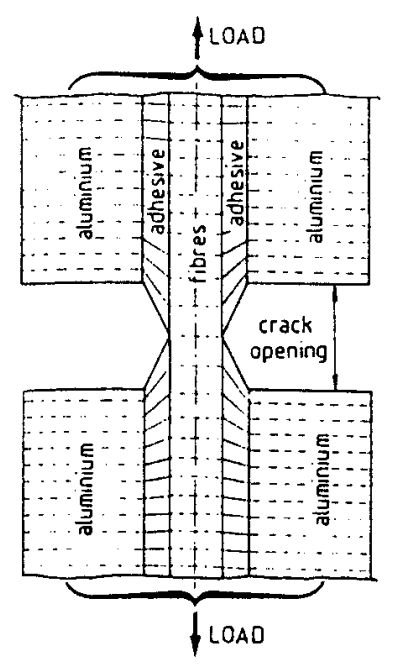

Figure 2.2: Adhesive Shear Deformation upon Crack Opening, Marissen (1989).

The delamination between the fiber and metal layers is an important mechanism in the crack bridging of fiber-metal laminates. Fiber bridging in fiber-metal laminates is different from crack-face bridging in conventional composites because the delamination has a large influence on the bridging stress. Delamination causes stress redistribution ahead and behind the crack tip that permits fibers to remain intact in the wake of the crack tip, Wilson and Wilson (1991). The delamination shape and size are related to the crack length and magnitude of the bridging stress.

An increase in delamination causes a decreasing fiber effectiveness and increasing stress intensity and crack growth rate in the metal layer, Marissen (1994). The fiber bridging stress is transferred to the cracked metal layer through the interface so a higher bridging stress will result in a higher driving force for delamination and consequently a higher delamination growth rate, Lin and Kao (1996). But with an 
increasing delamination, the bridging stress and adhesive shear stress are reduced and the resulting low bridging fiber stress will slow delamination growth but increase crack growth, Marissen (1991). Thus, it can be seen that the delamination zone is strongly dependent on the bridging stress.

The shape of the delamination zone was observed to be generally elliptical. Using ultrasonic scanning of specimens, several studies revealed an elliptical delamination shape, Lin and Kao (1996), Marissen (1991), and Takamatsu, et al. (1999). A triangular shape was considered by Guo and Wu (1998) in their work with CCT specimens made of GLARE. In another study, Guo and Wu (1999) stated that the delamination shapes are irregular during fatigue and not elliptical in most cases but closer to a triangle. A general delamination shape is shown in Figure 2.3 where a crack from a notch is bridged in the delaminated area.

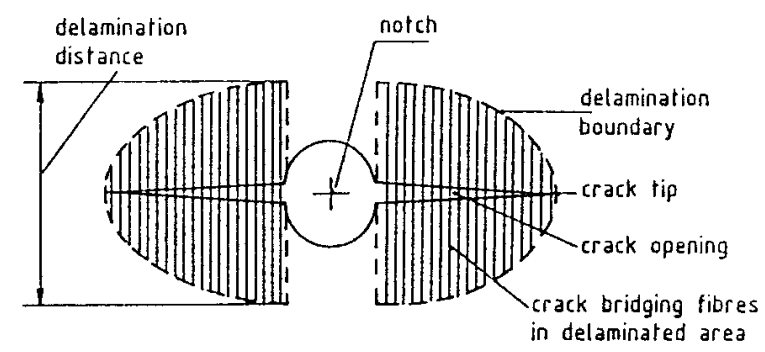

\section{Figure 2.3: General Delamination Shape due to a Crack from a Notch, Marissen (1991).}

\subsection{Stress Intensity Approach}

The stress intensity approach has been used due to its simplicity in obtaining the crack tip stresses and stress intensity factor. The stress intensity factor at the crack tip can be expressed by,

$$
K_{t i p}=K_{a}-K_{b}
$$

For crack extension to occur,

$$
K_{\text {tip }} \geq K_{c}
$$

Here, $K_{a}$ is the applied stress intensity factor, $K_{b}$ is the stress intensity factor due to fibre bridging, and $K_{c}$ is the fracture toughness of the material. For a particular geometry, $K_{a}$ and $K_{c}$ can be obtained from published results and polynomial functions obtained from 
previous tests. $K_{b}$, on the other hand, depends on bridging tractions and the crack opening displacement (see Figure 2.4).
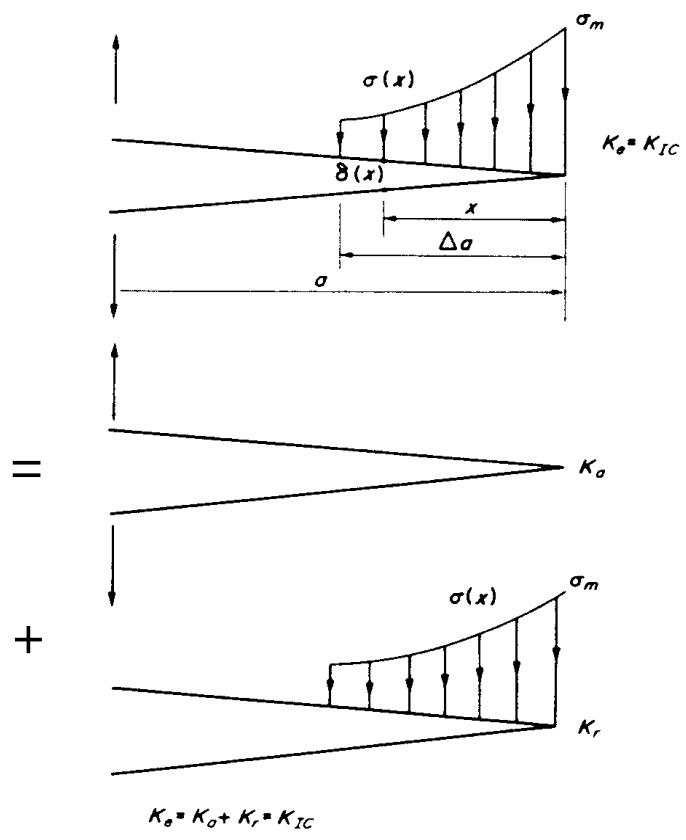

Figure 2.4: Superposition of Stress Intensity Factors for a Bridged Crack, Foote, et al. (1986).

\subsubsection{Power-Law Expressions for Bridging Stress}

In order to obtain the critical parameters that characterize the fibre-bridging process in fibre-metal laminates, it is essential to find the bridging stress distribution on the crack faces. Analytical expressions were proposed by Foote, et al. (1986) which were based on their work using strain-softening materials. An empirical power law relationship for the bridging stress distribution as a function of crack opening displacement was given by,

$$
\sigma_{B}(u)=\sigma_{B}^{*}\left[1-\frac{u(x)}{u^{*}}\right]^{m_{i}}
$$

Here, $u^{*}$ and $\sigma_{\mathrm{B}}{ }^{*}$ are the opening displacement and the bridging stress, respectively, at the fibre furthest from the crack tip, and $m_{i}$ is a power index. This gives a distribution of increasing crack-face bridging stress toward the crack tip. 
Suzuki and Sakai (1994) later used a relationship which gave a decreasing bridging stress toward the crack tip with another power index, $n_{d}$. The equation for this bridging stress is given as,

$$
\sigma_{B}(u)=\sigma_{B}^{*}\left[\frac{u(x)}{u^{*}}\right]^{n_{d}}
$$

As it is convenient to express the bridging stress distribution as a function of distance along the crack in the form of a power law, the relationship between the crack opening displacement and distance may also be similarly assumed. The crack opening displacement in terms of the bridging zone length, $b$, can be written as,

$$
u(x)=u^{*}\left(\frac{x}{b}\right)^{q}
$$

Cain and Tan (1997) modified equation (2.3) to define $\sigma_{\mathrm{B}}{ }^{*}$ in the same manner as equation (2.4) such that,

$$
\sigma_{B}(u)=\sigma_{B}^{*}\left[1+\left(1-\frac{u(x)}{u^{*}}\right)^{m_{i}}\right]
$$

The use of equations (2.4) and (2.6) with (2.5) yields new expressions with combined power indices ( $m$ and $n$ ) such that the respective expressions for $\sigma_{\mathrm{B}}$ as a function of distance $x$ from the crack tip become:

$$
\sigma_{B}(x)=\sigma_{B}^{*}\left(\frac{x}{b}\right)^{n}
$$

for stresses decreasing towards the crack-tip, and:

$$
\sigma_{B}(x)=\sigma_{B}^{*}\left[1+\left(1-\frac{x}{b}\right)^{m}\right]
$$

for stresses increasing towards the crack-tip (see Figure 2.5). 

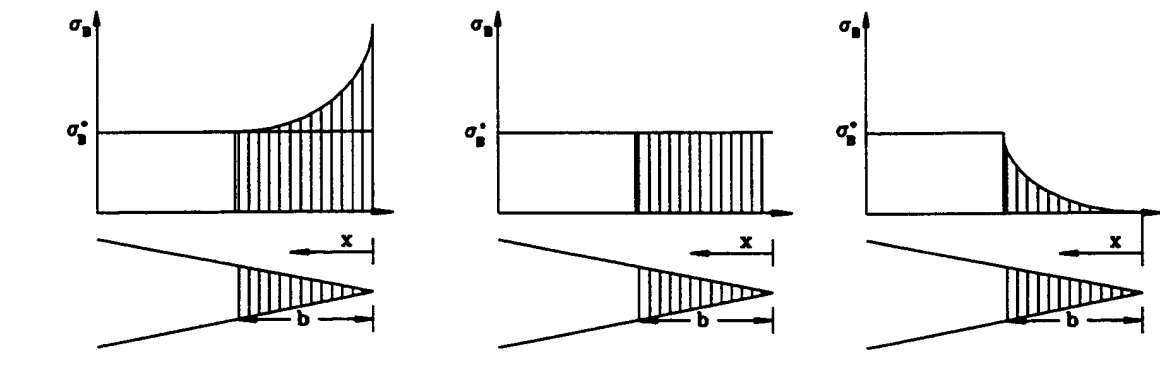

$$
\sigma_{B}(x)=\sigma_{B}^{*}\left[1+(1-(x / b))^{m}\right] \quad \sigma_{B}(x)=\sigma_{B}^{*} \quad \sigma_{B}(x)=\sigma_{B}^{*}(x / b)^{n}
$$

\section{Figure 2.5: Power-Law Bridging Stress Distributions}

The bridging stress distribution can be obtained once the magnitude of the stress in the last intact fibre away from the crack tip is known. This stress depends on the extend of debond between the fibre which it acts through, and the matrix. It can be determined by considering the strain in this fibre, Cain and Tan (1997). This strain, $\varepsilon^{*}$, is related to the half-crack opening displacement $v^{*}$ and the delamination length between the layers, $d$, at that position by:

$$
\varepsilon^{*}=\frac{v^{*}}{d}
$$

It can be seen that the bridging stress distribution in fibre-metal laminates is a three-dimensional problem. However, it can be treated as a two-dimensional one in the plane of the laminate by homogenization of mechanical properties through the thickness of the laminate. Although the bridging stress only acts on the fiber layer crack faces, it was assumed that it acts as a crack-closure stress all through the thickness, Cain and Tan (1997). The net force in the two-dimensional model must be the same as in the three-dimensional model and thus in the two-dimensional analysis, the stress in the last intact fiber farthest from the crack tip is:

$$
\sigma_{B}^{*}=E_{11}\left(\frac{A}{A_{T}}\right) \varepsilon^{*}
$$

$E_{11}$ is the Young's modulus in the fibre direction of the fibre laminae, $A$ is the crosssectional area of the laminae, and $A_{T}$ is the total cross-sectional area of the whole laminate. Combining equations (2.9) and (2.10) yields the expression: 


$$
\sigma_{B}^{*}=E_{11}\left(\frac{A}{A_{T}}\right)\left(\frac{v^{*}}{d}\right)
$$

It is shown here that in order to obtain the bridging stress distribution along the crack length, the half-crack opening displacement and the bridging stress have to be known at the last intact fibre from the crack tip; neither is known a priori. Hence, the procedure to obtain $\sigma_{\mathrm{B}}{ }^{*}$ is an iterative one. The maximum half crack opening displacement with no crack bridging, $v^{*}$ is first obtained using a numerical approach such as the finite element or the boundary element method. Using equation (2.11), the stress in the farthest intact fibre from the crack tip, $\sigma_{\mathrm{B}}{ }^{*}$ is then calculated and used in equations (2.7) and (2.8) to find the bridging stress distribution. A new $v^{*}$ is found in a subsequent numerical stress analysis. This procedure is then repeated until the values of $v^{*}$ and $\sigma_{\mathrm{B}}{ }^{*}$ converge to set tolerances.

For a uniform crack bridging stress distribution, where $n=m=0$, the distribution can be calculated directly if the half crack opening displacement without any crack bridging is known. Let the half-crack opening displacement at the crack mouth be

$$
v_{m}^{*}=\bar{v}^{*}
$$

By using Bueckner's superposition principle, Cain and Tan (1997) obtained the half crack opening displacement for a uniformly distributed crack bridging stress under an applied stress $\sigma$ as,

$$
v_{m}^{*}=\left(\frac{\sigma-\sigma_{B}}{\sigma}\right) \bar{v}^{*}
$$

Using equation (2.11), the uniform bridging stress distribution can be shown to be,

$$
\sigma_{B}=\frac{E_{11}\left(\frac{A}{A_{T}}\right)\left(\frac{\bar{v}^{*}}{d}\right)}{1+\frac{E_{11}}{\sigma}\left(\frac{A}{A_{T}}\right)\left(\frac{\bar{v}^{*}}{d}\right)}
$$




\subsection{Review of the Boundary Integral Equation (BIE) Method}

The boundary integral equation (BIE) method, also commonly known as the boundary element method (BEM), is an alternative to the finite element method (FEM) for the numerical solution of many engineering problems. Boundary element analysis is efficient in treating problems with stress concentrations and regions with high stress gradients such as those found near cracks. The use of fewer elements than in FEM is possible because only the boundary of the solution domain is discretized. As a result, the BIE method has become widely used in the study of fracture and fatigue of engineering components.

\subsection{The BIE Method for Anisotropic Elasticity}

The derivation of the BIE for anisotropic elasticity follows similar steps as that for the isotropic case. The difference lies in the stress-strain relations and the form of the fundamental solution, $U_{j i}(P, Q)$ and $T_{j i}(P, Q)$. Following the usual limiting process, the boundary integral equation for elastostatics can be written as, (see, e.g. Tan (1987)):

$$
\begin{aligned}
& C_{j i}(P) u_{i}(P)+\int_{S} u_{i}(Q) T_{j i}(P, Q) d S(Q)= \\
& \int_{S} t_{i}(Q) U_{j i}(P, Q) d S(Q)
\end{aligned}
$$

where

$$
C_{j i}(P)=\lim _{\varepsilon \rightarrow 0} \int_{S_{\varepsilon}} T_{j i}(P, Q) d S(Q)
$$

$C_{j i}(P)$ depends on the local geometry of the surface at point $P$, and $S_{\varepsilon}$ is the boundary region around $P . \quad T_{j i}(P, Q)$ and $U_{j i}(P, Q)$ represent the tractions and displacements, respectively, in the $x_{i}$-direction at $Q(x)$ due to the application of a unit concentrated load in the $x_{j}$-direction at $P(x)$ in a plane homogeneous infinite body.

The stress-strain relations for a two-dimensional anisotropic material in plane stress are:

$$
\left\{\begin{array}{c}
\varepsilon_{11} \\
\varepsilon_{22} \\
2 \varepsilon_{12}
\end{array}\right\}=\left[\begin{array}{lll}
a_{11} & a_{12} & a_{16} \\
a_{12} & a_{22} & a_{26} \\
a_{16} & a_{26} & a_{66}
\end{array}\right]\left\{\begin{array}{l}
\sigma_{11} \\
\sigma_{22} \\
\sigma_{12}
\end{array}\right\}
$$

Here $a_{i j}$ are the elastic compliances related to the engineering constants by, see, e.g. Tan and Gao (1992b): 


$$
\begin{aligned}
& a_{11}=\frac{1}{E_{11}} \quad a_{22}=\frac{1}{E_{22}} \quad a_{12}=-\frac{v_{12}}{E_{11}}=-\frac{v_{21}}{E_{22}} \\
& a_{16}=\frac{\eta_{12,1}}{E_{11}}=\frac{\eta_{1,12}}{G_{12}} \quad a_{26}=\frac{\eta_{12,2}}{E_{22}}=\frac{\eta_{2,12}}{G_{12}}
\end{aligned}
$$

$E_{11}$ and $E_{22}$ are Young's moduli in the principal material axis directions, $v_{i j}$ are Poisson's ratios, $G_{12}$ is the shear modulus, and $\eta_{i j, i}$ and $\eta_{i, i j}$ are the coefficients of mutual influence. The coefficients of mutual influence, $\eta_{i, i}$ and $\eta_{i, i j}$, are both zero for the case of orthotropy.

The characteristic equation derived by Lekhnitskii (1963) can be written as:

$$
a_{11} \mu^{4}-a_{16} \mu^{3}+\left(2 a_{12}+a_{66}\right) \mu^{2}-a_{26} \mu+a_{22}=0
$$

The roots of this equation are distinct and never real as long as the material is nonisotropic. These roots are required for the fundamental solution for anisotropic elasticity. The fundamental solutions for displacements and tractions, respectively, are now given by, see, e.g. Tan and Gao (1992b):

$$
\begin{aligned}
U_{j i}= & \left.2 \operatorname{Re} \mid r_{i 1} A_{j 1} \log _{e}\left(z_{1}\right)+r_{i 2} A_{j 2} \log _{e}\left(z_{2}\right)\right] \\
T_{j 1}= & 2 n_{1} \operatorname{Re}\left[\mu_{1}^{2}\left(\frac{A_{j 1}}{z_{1}}\right)+\mu_{2}^{2}\left(\frac{A_{j 2}}{z_{2}}\right)\right] \\
& -2 n_{2} \operatorname{Re}\left[\mu_{1}\left(\frac{A_{j 1}}{z_{1}}\right)+\mu_{1}\left(\frac{A_{j 2}}{z_{2}}\right)\right] \\
T_{j 2}= & -2 n_{1} \operatorname{Re}\left[\mu_{1}\left(\frac{A_{j 1}}{z_{1}}\right)+\mu_{2}\left(\frac{A_{j 2}}{z_{2}}\right)\right] i, j=1,2 \\
& +2 n_{2} \operatorname{Re}\left[\frac{A_{j 1}}{z_{1}}+\frac{A_{j 2}}{z_{2}}\right]
\end{aligned}
$$

where

$$
\begin{aligned}
& r_{1 j}=a_{11} \mu_{j}^{2}+a_{12}-a_{16} \mu_{j} \\
& r_{2 j}=a_{12} \mu_{j}+\frac{a_{22}}{\mu_{j}}-a_{26}
\end{aligned}
$$


and $n_{j}$ are the unit outward normal components at $Q$ with respect to the $x_{1}-x_{2}$ co-ordinate system. The complex constants $A_{j i}$ may be obtained from the following equations:

$$
\left[\operatorname{Im}\left\{B_{1}\right\} \quad \operatorname{Re}\left\{B_{1}\right\} \quad \operatorname{Im}\left\{B_{2}\right\} \quad \operatorname{Re}\left\{B_{2}\right\}\right]\left[\begin{array}{c}
\operatorname{Re}\left\{A_{j 1}\right\} \\
\operatorname{Im}\left\{A_{j 1}\right\} \\
\operatorname{Re}\left\{A_{j 2}\right\} \\
\operatorname{Im}\left\{A_{j 2}\right\}
\end{array}\right]=\left[\begin{array}{c}
-1 / 4 \pi \delta_{j 2} \\
1 / 4 \pi \delta_{j 1} \\
0 \\
0
\end{array}\right]
$$

where

$$
\left\{B_{i}\right\}=\left[\begin{array}{llll}
i & \mu_{i} & r_{1 i} & r_{2 i}
\end{array}\right]^{T}
$$

Equation (3.1) is an integral constraint equation relating the boundary tractions to the boundary displacements. These equations are, in general, intractable analytically and therefore numerical methods must be employed for their solution.

\subsubsection{Numerical Solution of the BIE}

The BIE is solved numerically by dividing the boundary $S$, of the solution domain into a series of $M$ line elements as shown in Figure 3.2. The geometry of the elements and the variation of the BIE functions over the elements are assumed to be quadratic. The formulation for the numerical procedure has been presented by Tan and Gao (1992a) (1992b), and Tan, et al. (1992). The quadratic isoparametric elements, as shown in Figure 3.3, are each defined by three equally spaced nodes with intrinsic coordinates $\zeta=-1, \zeta=0, \zeta=+1$, respectively.

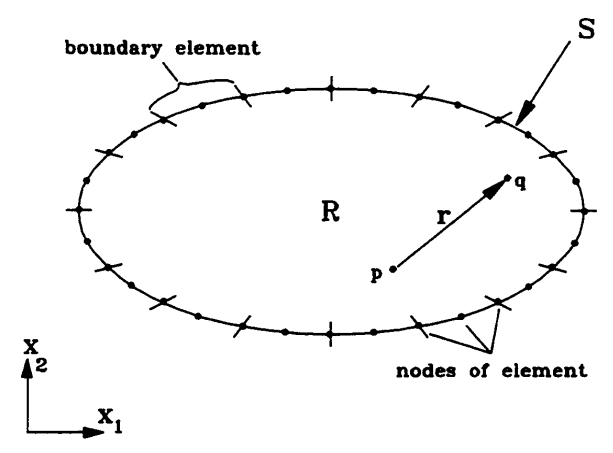

Figure 3.2: Solution domain $R$, with boundary $S$, divided into $M$ elements. 


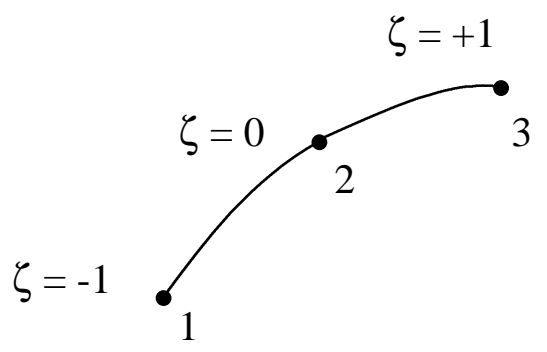

Figure 3.3: Quadratic one-dimensional element.

The shape functions for these elements are:

$$
\begin{array}{ll}
N^{(1)}(\zeta)=-\frac{1}{2} \zeta(1-\zeta) & \\
N^{(2)}(\zeta)=1-\zeta^{2} & \\
N^{(3)}(\zeta)=\frac{1}{2} \zeta(1+\zeta) & -1 \leq \zeta \leq+1
\end{array}
$$

The superscripts on the shape functions $N(\zeta)$ represent the local nodes of the element.

The geometry, displacements and tractions can then be expressed in terms of the shape functions and nodal values as:

$$
\begin{array}{ll}
x_{j}(\zeta)=N^{c}(\zeta) x_{j}^{c} & \\
u_{j}(\zeta)=N^{c}(\zeta) u_{j}^{c} & \\
t_{j}(\zeta)=N^{c}(\zeta) t_{j}^{c} & \mathrm{c}=1,3
\end{array}
$$

The discretised form of the boundary integral equations becomes:

$$
\begin{gathered}
C_{j i}\left(P^{a}\right) u_{i}\left(P^{a}\right)+\sum_{b=1}^{M} \sum_{c=1}^{3} u_{i}\left(P^{d(b, c)}\right) \int_{S} T_{j i}\left(P^{a}, Q\right) N^{c}(\zeta)|J(\zeta)| d S \\
=\sum_{b=1}^{M} \sum_{c=1}^{3} t_{i}\left(P^{d(b, c)}\right) \int_{S} U_{j i}\left(P^{a}, Q\right) N^{c}(\zeta)|J(\zeta)| d S \\
P^{a}=1, N
\end{gathered}
$$


This gives a total of $4 M$ linear algebraic equations for the unknown displacements and tractions at the boundary nodes of the solution domain where,

$$
\begin{aligned}
& \mathrm{M}=\text { number of elements in the mesh } \\
& \mathrm{N}=\text { number of nodes }=2 \mathrm{M} \\
& \mathrm{P}^{\mathrm{d}(\mathrm{b}, \mathrm{c})}=\mathrm{c}^{\text {th }} \text { node of the } \mathrm{b}^{\text {th }} \text { element }=1, \mathrm{~N} \\
& \mathrm{~b}=1, \mathrm{M} \\
& \mathrm{c}=1,3 \\
& |J(\zeta)|=\frac{d S}{d \zeta}=\text { Jacobian of transformation }
\end{aligned}
$$

Standard matrix methods can then be used to solve for the unknown displacements and tractions on the boundary, $\mathrm{S}$.

\subsection{Linear-Elastic Fracture Mechanics Analysis by the BIE Method}

The stress intensity factor characterizes the crack tip stress field in a linear elastic material. The aim of fracture mechanics analysis is to calculate this parameter from the measured displacements and stresses near the crack tip which can then be compared to the fracture toughness of the material to establish structural integrity. Figure 3.4 defines the coordinate system.

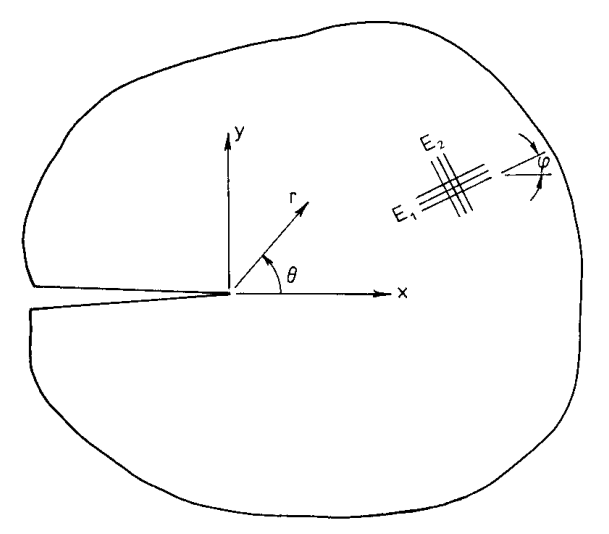

Figure 3.4: Co-ordinate system and material orientation in LEFM analysis.

The stress state in terms of the stress intensity factors in anisotropic elasticity is the same as in isotropic elasticity; the stresses are given by: 


$$
\begin{aligned}
& \sigma_{y y}=K_{I} / \sqrt{2 \pi r} \\
& \sigma_{x y}=K_{I I} / \sqrt{2 \pi r}
\end{aligned}
$$

The displacements on the crack faces $\left(\theta=180^{\circ}\right.$ in Figure 3.4) in terms of the stress intensity factors are, see, e.g. Tan and Gao (1992a):

$$
\left\{\begin{array}{l}
u_{x} \\
u_{y}
\end{array}\right\}=\sqrt{\frac{2 r}{\pi}}\left[\begin{array}{ll}
D_{11} & D_{12} \\
D_{21} & D_{22}
\end{array}\right]\left\{\begin{array}{l}
K_{I} \\
K_{I I}
\end{array}\right\}
$$

where

$$
\begin{array}{ll}
D_{11}=\operatorname{Im}\left[\frac{s_{2} p_{1}-s_{1} p_{2}}{s_{1}-s_{2}}\right] & D_{12}=\operatorname{Im}\left[\frac{p_{1}-p_{2}}{s_{1}-s_{2}}\right] \\
D_{21}=\operatorname{Im}\left[\frac{s_{2} q_{1}-s_{1} q_{2}}{s_{1}-s_{2}}\right] & D_{22}=\operatorname{Im}\left[\frac{q_{1}-q_{2}}{s_{1}-s_{2}}\right]
\end{array}
$$

The material property parameters, $s_{j}$, given in Tan and Gao (1992a), are related to the roots of the characteristic equation, $\mu_{j}$ by:

$$
s_{j}=\frac{\mu_{j} \cos \phi+\sin \phi}{\cos \phi-\mu_{j} \sin \phi} \quad j=1,2
$$

and $p_{j}$ and $q_{j}$ are:

$$
\begin{aligned}
& p_{j}=a_{11}^{\prime} s_{j}^{2}+a_{12}^{\prime} s_{j} \\
& q_{j}=\frac{a_{12}^{\prime} s_{j}^{2}+a_{22}^{\prime}-a_{26}^{\prime} s_{j}}{s_{j}} \quad j=1,2
\end{aligned}
$$

The local compliances are obtained from the following transformation from the global $\mathrm{x}_{1}-\mathrm{x}_{2}$ co-ordinate system to the local Cartesian system by:

$$
\begin{aligned}
a_{11}^{\prime}= & a_{11} \cos ^{4} \phi+\left(2 a_{12}+a_{66}\right) \sin ^{2} \phi \cos ^{2} \phi+a_{22} \sin ^{4} \phi+ \\
& \left(a_{16} \cos ^{2} \phi+a_{26} \sin ^{2} \phi\right) \sin 2 \phi \\
a_{22}^{\prime}= & a_{11} \sin ^{4} \phi+\left(2 a_{12}+a_{66}\right) \sin ^{2} \phi \cos ^{2} \phi+a_{22} \cos ^{4} \phi- \\
& \left(a_{16} \cos ^{2} \phi+a_{26} \sin ^{2} \phi\right) \sin 2 \phi
\end{aligned}
$$




$$
\begin{aligned}
a_{12}^{\prime}= & a_{12}+\left(a_{11}+a_{22}-2 a_{12}-a_{66}\right) \sin ^{2} \phi \cos ^{2} \phi+ \\
& \frac{1}{2}\left(a_{26}-a_{16}\right) \sin 2 \phi \cos 2 \phi \\
a_{66}^{\prime}= & a_{66}+2\left(a_{11}+a_{22}-2 a_{12}-a_{66}\right) \sin ^{2} 2 \phi+ \\
& 2\left(a_{26}-a_{16}\right) \sin 2 \phi \cos 2 \phi \\
a_{16}^{\prime}= & {\left[a_{22} \sin ^{2} \phi-a_{11} \cos ^{2} \phi+\frac{1}{2}\left(2 a_{12}+a_{66}\right) \cos 2 \phi\right] \sin 2 \phi+} \\
& \left(a_{16} \cos ^{2} \phi\right)\left(\cos ^{2} \phi+3 \sin ^{2} \phi\right)+\left(a_{16} \sin ^{2} \phi\right)\left(3 \cos ^{2} \phi-\sin ^{2} \phi\right) \\
a_{26}^{\prime}= & {\left[a_{22} \sin ^{2} \phi-a_{11} \cos ^{2} \phi+\frac{1}{2}\left(2 a_{12}+a_{66}\right) \cos 2 \phi\right] \sin 2 \phi+} \\
& \left(a_{16} \sin ^{2} \phi\right)\left(\cos ^{2} \phi-\sin ^{2} \phi\right)+\left(a_{26} \cos ^{2} \phi\right)\left(\cos ^{2} \phi-3 \sin ^{2} \phi\right)
\end{aligned}
$$

\subsubsection{Quarter-Point Crack-Tip Element in Plane Orthotropic Bodies}

The expressions for the stresses and displacements in the vicinity of the cracktip are proportional to $1 / \sqrt{r}$ and $\sqrt{r}$, respectively. In order to obtain the proper variation of the displacements, the mid-point node of the element adjacent to the crack tip can be shifted to the quarter-point position as shown in Figure 3.5. This results in the proper $\sqrt{r}$ variation for the displacement field. In order to obtain the variation of the tractions, the nodal shape functions associated with nodal tractions have to be multiplied by $1 / \sqrt{r}$, where $l$ is the length of the element. Details of this formulation have been presented by Tan and Gao (1992a), Martinez and Dominguez (1984), and Cruse and Wilson(1977).

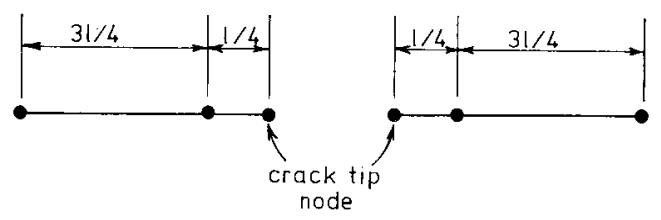

Figure 3.5: Quarter-point crack-tip element of length, $l$. 
The displacement variation in this quarter-point element can be expressed in terms of nodal values as:

$$
u_{j}=u_{j}^{A}+\left(-u_{j}^{C}+4 u_{j}^{B}-3 u_{j}^{A}\right)\left(\frac{r}{l}\right)^{1 / 2}+\left(2 u_{j}^{C}-4 u_{j}^{B}+2 u_{j}^{A}\right)\left(\frac{r}{l}\right)
$$

where the superscripts in $u_{j}$ denote the nodes as shown in Figure 3.1. The analytical expressions for $K_{I}$ and $K_{I I}$ based on the nodal displacements may be obtained by equating the coefficients of $\sqrt{r}$ in equations (3.16) and (3.27). If the crack lies parallel to the global $x_{1}$-axis, the expressions may be written as:

$$
\left[\begin{array}{l}
K_{I} \\
K_{I I}
\end{array}\right]=\frac{\sqrt{\pi / 2 l}}{\operatorname{Det}[D]}\left[\begin{array}{cc}
D_{22} & -D_{12} \\
-D_{21} & D_{22}
\end{array}\right]\left[\begin{array}{l}
-u_{1}^{C}+4 u_{1}^{B}-3 u_{1}^{A} \\
-u_{2}^{C}+4 u_{2}^{B}-3 u_{2}^{A}
\end{array}\right]
$$

where

$$
\operatorname{Det}[D]=\left(D_{11} D_{22}-D_{21} D_{12}\right)
$$

This equation is commonly referred to as the 'displacement formula' in BEM analysis.

The computed traction values $\bar{t}_{j}$ are related to the physical traction values $t_{j}$ at the nodes, again denoted by the superscripts, by:

$$
\begin{aligned}
& \bar{t}_{j}^{E}=t_{j}^{E} \\
& \bar{t}_{j}^{D}=\frac{1}{2} t_{j}^{D} \\
& \bar{t}_{j}^{A}=\lim _{r \rightarrow 0} t_{j}^{A} \sqrt{\frac{r}{l}}
\end{aligned}
$$

If the crack lies parallel to the global $x_{l}$-axis, then the tractions with the incorporated singularity at the crack-tip are related to the stress intensity factors by:

$$
\begin{aligned}
& \bar{t}_{1}^{A}=\lim _{r \rightarrow 0} t_{1}^{A} \sqrt{\frac{r}{l}}=\lim _{r \rightarrow 0} \sigma_{12} \sqrt{\frac{r}{l}}=\frac{K_{I I}}{\sqrt{2 \pi l}} \\
& \bar{t}_{2}^{A}=\lim _{r \rightarrow 0} t_{2}^{A} \sqrt{\frac{r}{l}}=\lim _{r \rightarrow 0} \sigma_{22} \sqrt{\frac{r}{l}}=\frac{K_{I}}{\sqrt{2 \pi l}}
\end{aligned}
$$

or

$$
\begin{aligned}
& K_{I}=\bar{t}_{2}^{A} \sqrt{2 \pi l} \\
& K_{I I}=\bar{t}_{1}^{A} \sqrt{2 \pi l}
\end{aligned}
$$


This equation is referred to as the 'traction formula' in BEM analysis. It is evident that the displacement formula involves additional computational effort as compared with the traction formula. Tan and Gao (1992a) also showed that the stress intensity factors obtained using the displacement formulas are significantly more sensitive to the size of the crack-tip elements used. The traction formula is more widely used and the stress intensity factors can be obtained directly from the computed nodal tractions. Although these equations have been derived for a case where the crack lies parallel to the global $x_{I^{-}}$axis, they remain valid for an arbitrarily oriented crack provided that the $x_{I^{-}}$and $x_{2^{-}}$ components of the displacements and tractions are replaced by the $x$ - and $y$-components in the local co-ordinate system.

\subsection{Analysis of Fully Bridged Cracked FMLs with Notches}

\section{Problem Definition and Scope}

Three different rectangular fibre-metal laminate plates with cracks emanating from the edge of a circular cut-out were analysed by the boundary element method (BEM) in two-dimensions and the finite element method (FEM) in three-dimensions. The rectangular plates were subjected to a remote uniform tension, $\sigma$, in the fibre direction and referring to Figure 4.1 they were (i) a plate with a semi-circular hole at one edge with a single crack emanating from it, (ii) a plate with a semi-circular hole at both edges with a crack emanating from each, and (iii) a plate with a central circular hole with two symmetric cracks emanating from it. The orientation of the plane of the cracks was chosen to be perpendicular to the fibre direction.

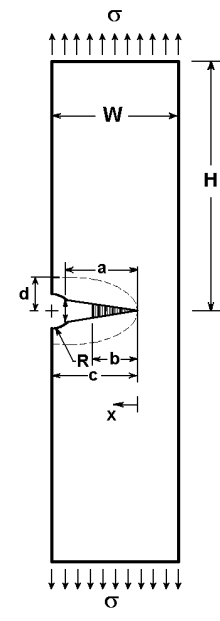

Problem (i)

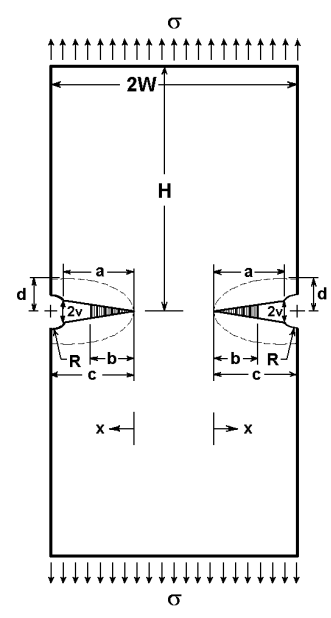

Problem (ii)

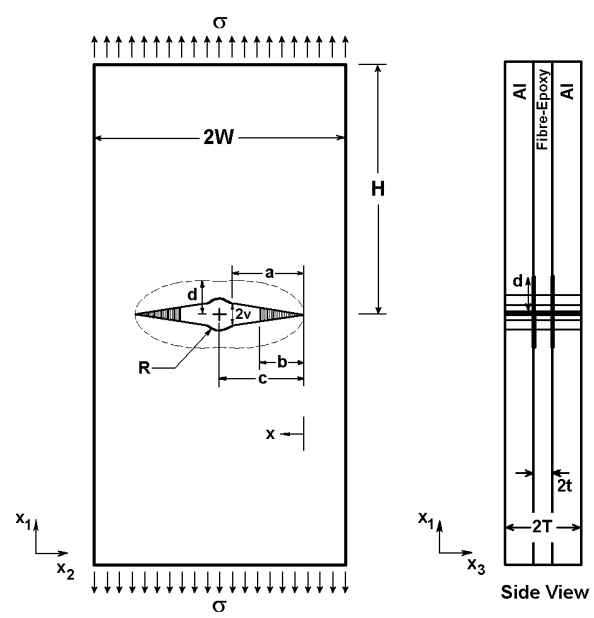

Problem (iii)

Figure 4.1: Cracked FML Geometries 
The geometry of the rectangular plates in the $x_{1}-x_{2}$ plane was defined by the height-to-width ratio, $H / 2 W=2$ for case (i), and $H / W=2$ for cases (ii) and (iii). The radius-to-width ratio of the circular cut-out for case (i) was chosen as $R / 2 W=0.1$, and $R / W=0.1$ for cases (ii) and (iii). The relative crack lengths considered for case (i) were $c / 2 W=0.2,0.3,0.4,0.5$, and for cases (ii) and (iii), were $c / W=0.2,0.3,0.4,0.5$. The height to fibre-reinforced laminae thickness ratio, $H / t$, and the total laminate to fibrereinforced laminae thickness ratio, $T / t$ were taken to be 400 and 4 , respectively. It was assumed to be elastic and that linear elastic fracture mechanics (LEFM) is applicable. Also, the thickness of the adhesive between the aluminum layer and the fibre layer was assumed to be small and have negligible influence on the material properties of the laminate. Furthermore, it was also assumed that there were no residual stresses in the laminate.

Two delamination zone shapes were considered in this study. One shape was that of a quarter-circle and the other shape was that of a quarter-ellipse with a majorminor axis ratio of 2, around each crack flank. These shapes were centred at the crack mouth of each crack, which corresponds to one or more edges of the hole. The delamination zone size, $d$, was taken at the last intact fibre away from the crack-tip in the bridged zone. For a fully bridged crack, the edge of the hole represents the last intact fibre away from the crack tip and hence $d$ was set equal to the crack length, $a$, for the circular delamination shape and $1 / a$ for the elliptical delamination shape. Experimentally observed delamination shapes are generally elliptical so a circular shape represents a more severe situation than would likely be encountered. Nevertheless, it is of interest to obtain the effect of an increased delamination size on the bridging stress and the stress intensity factor.

\section{Two-Dimensional Analysis}

The problem to be treated involved several parameters such as: the fatigue crack length, $a$, the equivalent crack length, $c$, the delamination zone size, $d$, and the power law indices, $n$ and $m$ of Eqns. (2.7) and (2.8). In the case of partial bridging, the bridging zone length, $b$ is also varied depending on the amount of bridging fibres present. For full bridging of the crack faces, $b$ is equal to the crack length, $a$.

The two-dimensional numerical stress analysis was carried out using the boundary element method (BEM) for anisotropic elasticity employing quadratic isoparametric elements, see Gao and Tan (1992b). The bridging stress distribution was assumed to be a continuous closing pressure on the crack faces according to Eqs. (2.7) and (2.8), with both of the power-law indices $n$ and $m$ having values of $0,0.5$ and 1.0. The crack was assumed to have propagated through both the metal layers and the fibre layer, and plane stress conditions were assumed here since the laminate is very thin through the thickness. One-quarter of each plate was modelled due to geometric and material symmetry about two axes. A typical BIE mesh is shown in Figure 4.2a. 


\section{Three-Dimensional Analysis}

The finite element method (FEM) was used for the three-dimensional stress analysis, employing two commercial software packages: SDRC I-DEAS ${ }^{\mathrm{TM}} 7.0$ used for the mesh generation, and MSC/Nastran ${ }^{\mathrm{TM}} 70.7$ used as the FE solver. The elements used for the FEM analysis were 20-noded quadratic iso-parametric solid bricks with the mid-side nodes of the elements adjacent to the crack-tip shifted to the quarter-point position. The 3-D analysis of a fully bridged fibre-metal laminate consisted of a crack propagating in the metal layers only with the fibre layer remaining crack free. Each plate in the 3-D analysis was modelled as one-eight of the whole plate due to geometric and material symmetry in three planes. A typical 3-D FEM mesh is shown in Figure $4.2 \mathrm{~b}$ and comparing it to the BEM mesh in Figure 4.2a, it can be seen that the modelling of the BEM mesh is far less time consuming than that for the FEM mesh.

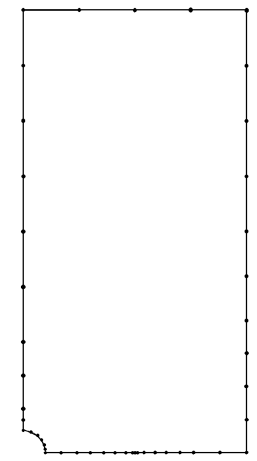

(a) BEM mesh - elements $=23$

$$
- \text { nodes }=46
$$

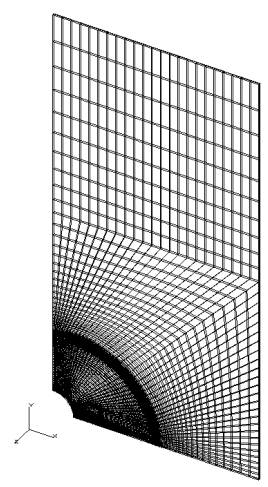

(b) FEM mesh - elements $=7716$

- nodes $=45555$

Figure 4.2: Typical BEM and FEM meshes; $c / W=0.5$, circular delamination.

\section{Material Properties}

The fibre-metal laminate material, ARALL2-2/1, was used in this study. ARALL2 is a hybrid composite consisting of alternating aluminum 2024-T3 layers with aramid-epoxy layers. The stacking is defined by the lay-up, which in this case is $2 / 1$ and signifies two layers of aluminum sandwiching one layer of fibre-epoxy.

The material properties of each layer are shown in Table 4.1 and the indices 1 , 2 , and 3 denote the fibre, transverse and through-the-thickness directions. In the 3-D FEM analysis, the aluminum layer was treated as isotropic and the fibre layers were treated as orthotropic with the following material property relations in the thickness direction: 


$$
\begin{array}{lll}
E_{33}=E_{22} & G_{13}=G_{12} & G_{23}=\frac{E_{22}}{2\left(1+v_{32}\right)} \\
v_{21}=v_{31} & v_{23}=v_{32} & -\frac{v_{21}}{E_{22}}=-\frac{v_{12}}{E_{11}}
\end{array}
$$

For the 2-D BEM modelling, the material properties were homogenized through the thickness of the laminate using classical laminate plate theory. The resulting homogenized material properties for ARALL that are used in the twodimensional BEM analysis are also listed in Table 4.1. The thickness of the aluminum layer was chosen as $0.3 \mathrm{~mm}$ and the thickness of the aramid-epoxy layers was chosen as $0.2 \mathrm{~mm}$.

Table 4.1: Material Properties for Aluminum, Aramid-Epoxy, and ARALL2-2/1. Yeh (1988).

\begin{tabular}{c|c|c|c|c|c|c} 
Material & $\begin{array}{c}\mathbf{E}_{1 \mathbf{1 1}} \\
(\mathrm{GPa})\end{array}$ & $\begin{array}{c}\mathbf{E}_{\mathbf{2 2}} \\
(\mathrm{GPa})\end{array}$ & $\begin{array}{c}\mathbf{G}_{\mathbf{1 2}} \\
(\mathrm{GPa})\end{array}$ & $\begin{array}{c}\mathbf{G}_{\mathbf{2 3}} \\
(\mathrm{GPa})\end{array}$ & $\mathbf{v}_{\mathbf{1 2}}$ & $\mathrm{v}_{\mathbf{2 3}}$ \\
\hline Aramid-Epoxy & 62.7 & 4.56 & 1.63 & 1.53 & 0.355 & 0.493 \\
\hline Aluminum & 71.7 & & 27.6 & & 0.300 & \\
\hline ARALL2-2/1 & 69.5 & 55.9 & 20.7 & & 0.300 &
\end{tabular}

\subsection{Results and Discussion}

The bridging stress and the stress intensity factor results are presented in this section for a fully bridged ARALL laminate with circular and elliptical delamination shapes. The bridging stress and stress intensity factor results obtained by a BEM analysis in two-dimensions are compared to those obtained from a three-dimensional FEM analysis.

\subsubsection{FEM Analysis}

\section{Bridging Stress Distribution}

The bridging stress, $\sigma_{\mathrm{B}}$, was taken along the mid-plane of the fibre layer in the finite element analysis and normalized with respect to the remote applied tensile stress, 
$\sigma$. The variations of the normalized bridging stress, $\sigma_{\mathrm{B}} / \sigma$, with relative distance $x / a=-$ 1.0 to $x / a=1.0$ from the crack-tip, along the centreline of the fibre layer for an edge crack, double-edge crack and central crack are shown in Figures 4.3 to 4.8. These geometries have circular and elliptical delamination shapes and varying normalized crack lengths, $c / W$, from 0.2 to 0.5 for the double-edge and central crack and $c / 2 W$ of 0.2 to 0.5 for the edge crack.

The normalized bridging stress, $\sigma_{\mathrm{B}} / \sigma$, for all crack geometries increases as $x / a$ approaches 0 and 1.0, corresponding to the crack-tip and crack mouth, respectively. For example, for a central crack with a circular delamination and crack length of $c / W=0.5$, the normalized bridging stress at $x / a=0$ is 4.24 . This stress decreases to about 2.05 at $x / a=0.53$ and increases to 10.14 at $x / a=1.0$. The increase of the normalized bridging stress at $x / a=0$ is likely due to the enforced singularity of the quarter-point crack-tip elements in the aluminum layer adjacent to the mid-point elements in the fibrereinforced layer of the mesh. The large increase of the normalized bridging stress, $\sigma_{\mathrm{B}} / \sigma$, at $x / a=1.0$, which represents the notch root, is due to the effect of stress concentration near the notch.

The results in Figures 4.3 to 4.5 show that for all crack lengths, plate geometries, and delamination zone shapes, the normalized bridging stress, $\sigma_{\mathrm{B}} / \sigma$, at the notch root (or crack mouth), represented by $x / a=1.0$, is very large compared to the normalized bridging stress anywhere else along the crack line. The highest normalized bridging stress, $\sigma_{\mathrm{B}} / \sigma$, for example, is for the edge crack with a circular delamination shape and crack length, $c / 2 W$ of 0.5 , where it reaches $\sigma_{\mathrm{B}} / \sigma=18.92$. The trends in these figures show that the fibres near the hole could be highly strained and would likely fail when their ultimate tensile stress is exceeded. The rapid increase of the normalized bridging stress, $\sigma_{\mathrm{B}} / \sigma$, near the hole is generally confined to a small region, typically 5 to $10 \%$ of the total crack length, where the stress concentration effects are the highest. Therefore, the presence of a notch causes the load through the fibres to be concentrated near the notch, which in turn greatly increases the bridging stresses in those fibres.

For the central crack and double edge crack in Figures 4.6 and 4.8, the normalized bridging stress distribution, $\sigma_{\mathrm{B}} / \sigma$, decreases with increasing crack length, $c / W$. This is opposite to the trend in fibre-metal laminates without notches for these two cracked geometries, where the bridging stress distribution increases with increasing crack length, Cain and Tan (1997). The decrease in the normalized bridging stress observed here can be explained by considering the influence of the stress concentration caused by the notch. For relatively small crack lengths compared to the size of the notch, the bridging stresses caused by the presence of the notch are higher than the bridging stresses caused by the crack alone, and the effect of the stress concentration slowly diminishes with increasing distance from the notch. 
B.E. Cudzilo and C. L. Tan / Electronic Journal of Boundary Elements, Vol. 1, No. 3, pp. 336-403 (2003)
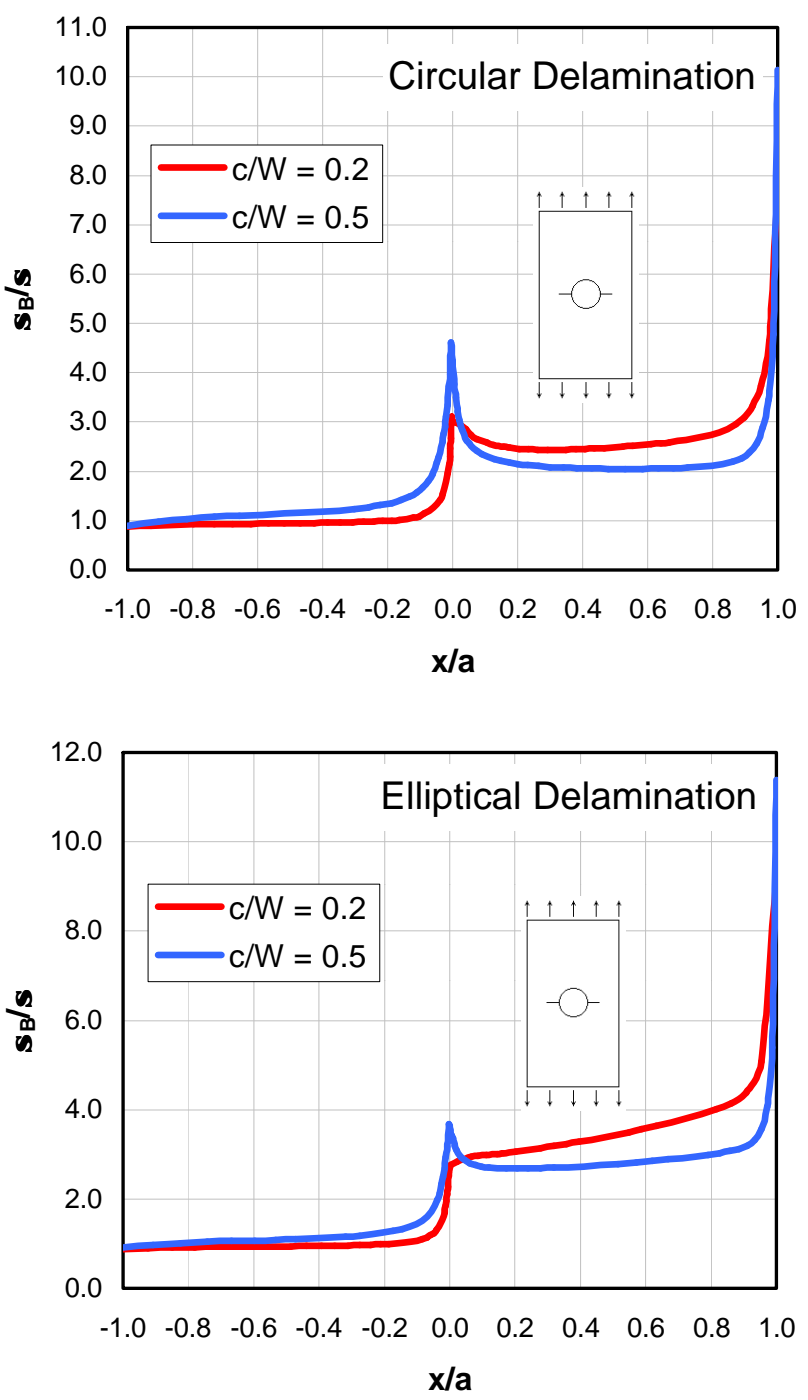

Figure 4.3: Variations of normalized bridging stress, $\sigma_{\mathrm{B}} / \sigma$, with relative distance from the crack-tip, x/a, along the centreline of the aramid-epoxy layer, for the fully bridged central crack problem with circular and elliptical delaminations. (FEM analysis) 
B.E. Cudzilo and C. L. Tan / Electronic Journal of Boundary Elements, Vol. 1, No. 3, pp. 336-403 (2003)
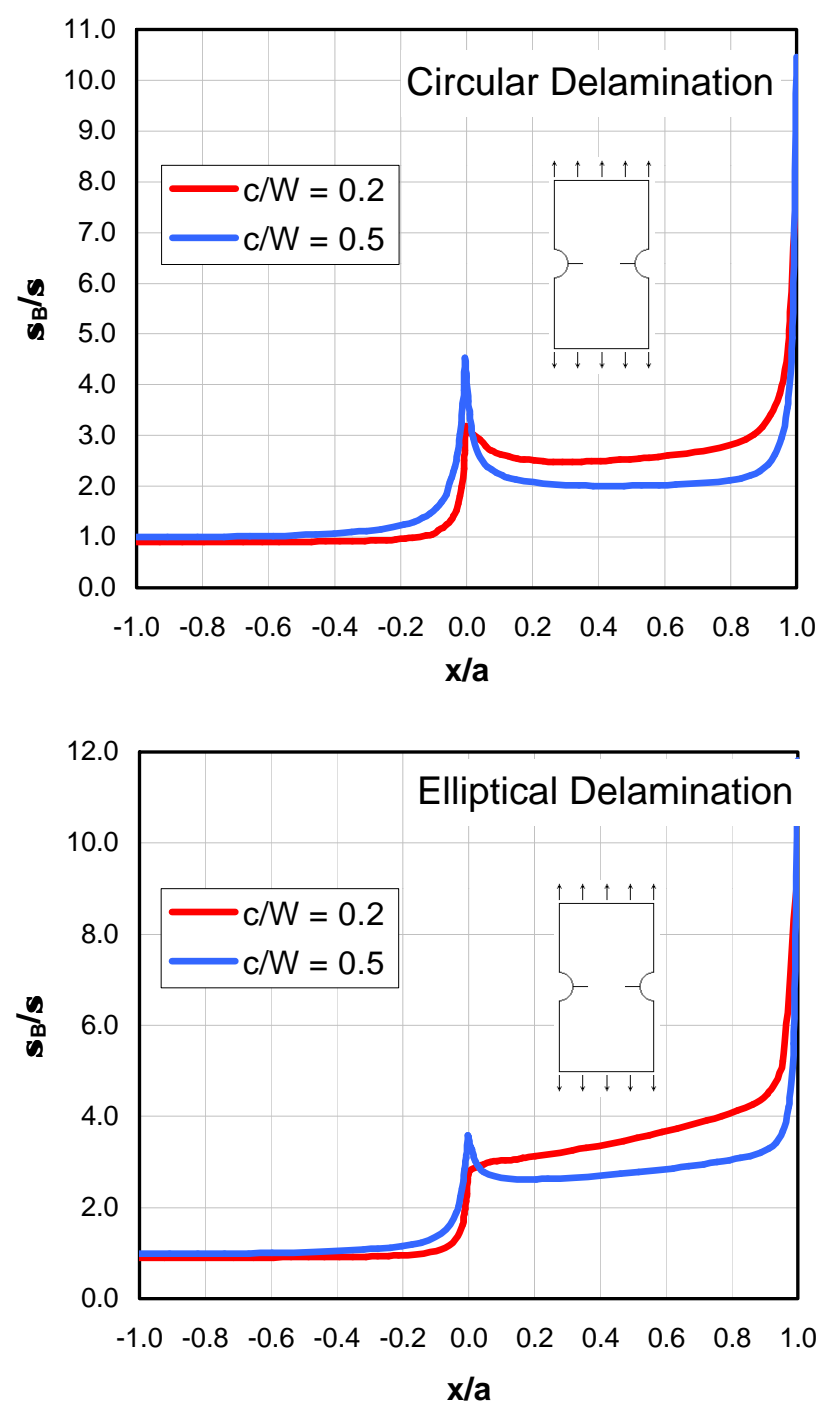

Figure 4.4: Variations of normalized bridging stress, $\sigma_{\mathrm{B}} / \sigma$, with relative distance from the crack-tip, x/a, along the centreline of the aramid-epoxy layer, for the fully bridged double edge crack problem with circular and elliptical delamination. (FEM analysis) 
B.E. Cudzilo and C. L. Tan / Electronic Journal of Boundary Elements, Vol. 1, No. 3, pp. 336-403 (2003)
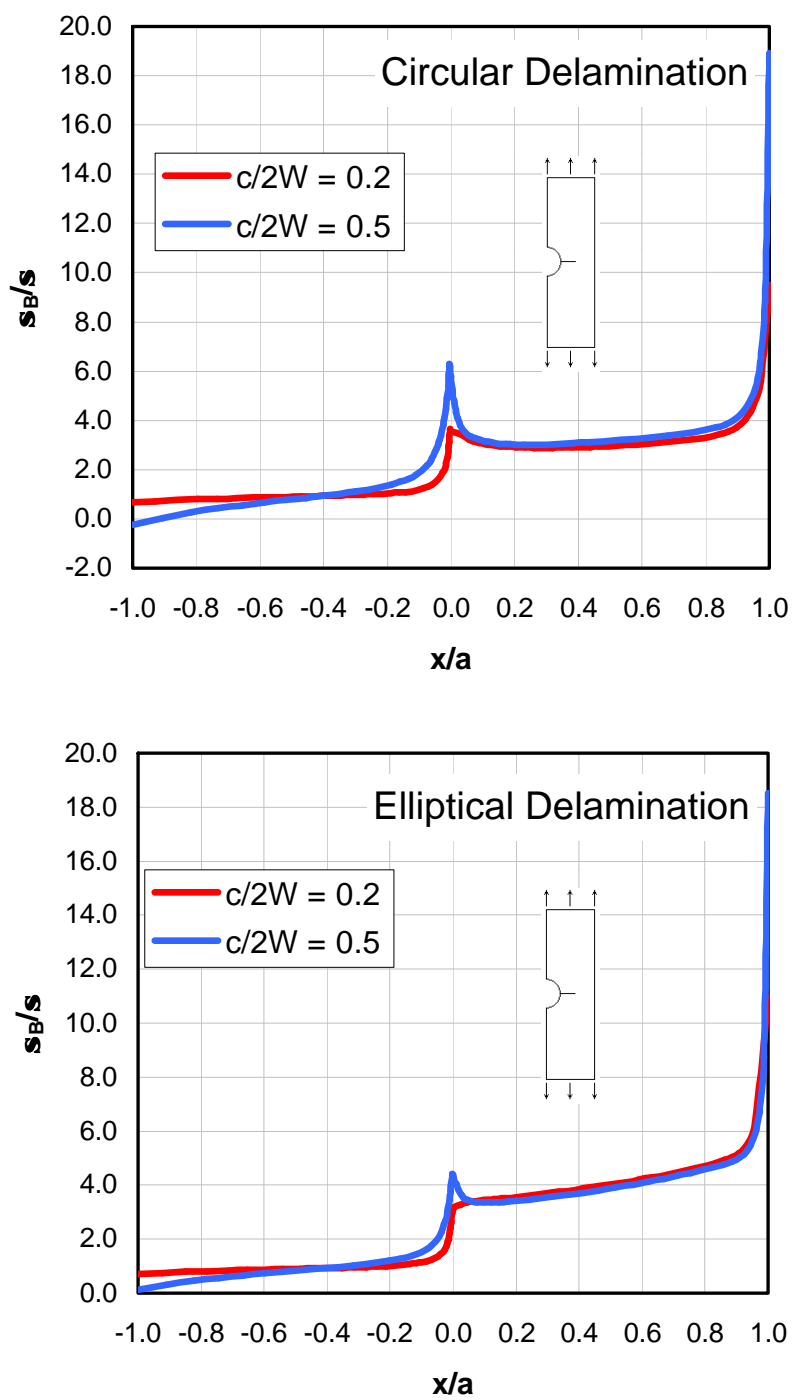

Figure 4.5: Variations of normalized bridging stress, $\sigma_{\mathrm{B}} / \sigma$, with relative distance from the crack-tip, x/a, along the centreline of the aramid-epoxy layer, for the fully bridged edge crack problem with circular and elliptical delamination. (FEM analysis) 
For example, for a double-edge crack with an elliptical delamination the normalized bridging stresses, $\sigma_{\mathrm{B}} / \sigma$, are highest for $c / W=0.2$ and lowest for $c / W=0.5$. The same trend is observed for a double-edge crack with circular delamination and a central crack with both delamination shapes. It is expected that the bridging stress distribution would begin to increase with increasing crack length far away from the notch and this is confirmed in Figures 4.6 and 4.7, where an inflexion point can be seen and the normalized bridging stress distribution, $\sigma_{\mathrm{B}} / \sigma$, near the crack-tip begins to increase with increasing crack length, $c / W$, since the effect of the crack on the stresses becomes dominant over that due to the notch.

For the edge crack in Figure 4.8, the bridging stress distribution is different from that for the double-edge and central cracks. The normalized bridging stress distribution, $\sigma_{\mathrm{B}} / \sigma$, for the edge crack increases with increasing crack length, $c / 2 W$ of 0.3 to 0.5 for both delamination shapes. This can be attributed to the effect of bending about the crack tip as a result of the unsymmetrical geometry caused by the presence of the crack and notch. The bending causes higher strains in the fibres with increasing distance from the crack tip, $x$, and hence the normalized bridging stress, $\sigma_{\mathrm{B}} / \sigma$ increase as the crack length, $c / 2 W$ increases. In this case, the effects on the bridging stress of the crack opening plus bending dominate over the effects on the bridging stress caused by the stress concentration near the notch. Also, there does not appear to be an inflexion of the bridging stress distributions for either the circular delamination or the elliptical delamination shape in the $x / a=0$ to $x / a=1.0$ region as was observed for the central and double-edge crack geometries. This signifies, in this case, that the bending effect of the edge crack geometry is greater than the effect of the stress concentration caused by the notch for the whole crack length, resulting in the observed distribution.

The claim of a dominant stress concentration effect over the whole crack length cannot be made for every delamination shape because the size of the delamination shape is another factor influencing the bridging stress distribution. For the circular delamination shape, the normalized bridging stress distribution, $\sigma_{\mathrm{B}} / \sigma$, away from the crack tip, for $c / 2 W=0.2$ is almost equal to the distribution for $c / 2 W=0.4$. For the elliptical delamination shape, the normalized bridging stress distribution, $\sigma_{\mathrm{B}} / \sigma$, away from the crack tip, for $c / 2 W=0.2$ is higher than that for $c / 2 W=0.5$. The reason for this can be explained by considering the effects of bending and stress concentration effects. The contribution of bending on bridging stress is much lower for shorter crack lengths, but the effects of stress concentration near the notch are much greater for shorter crack lengths. Therefore, the bending effect on the bridging stress distribution is sufficient to overcome the stress concentration effect, but only for relatively large crack lengths compared to the hole size. The bending effect on the magnitude of the bridging stress also appears to depend on the delamination shape. 
B.E. Cudzilo and C. L. Tan / Electronic Journal of Boundary Elements, Vol. 1, No. 3, pp. 336-403 (2003)
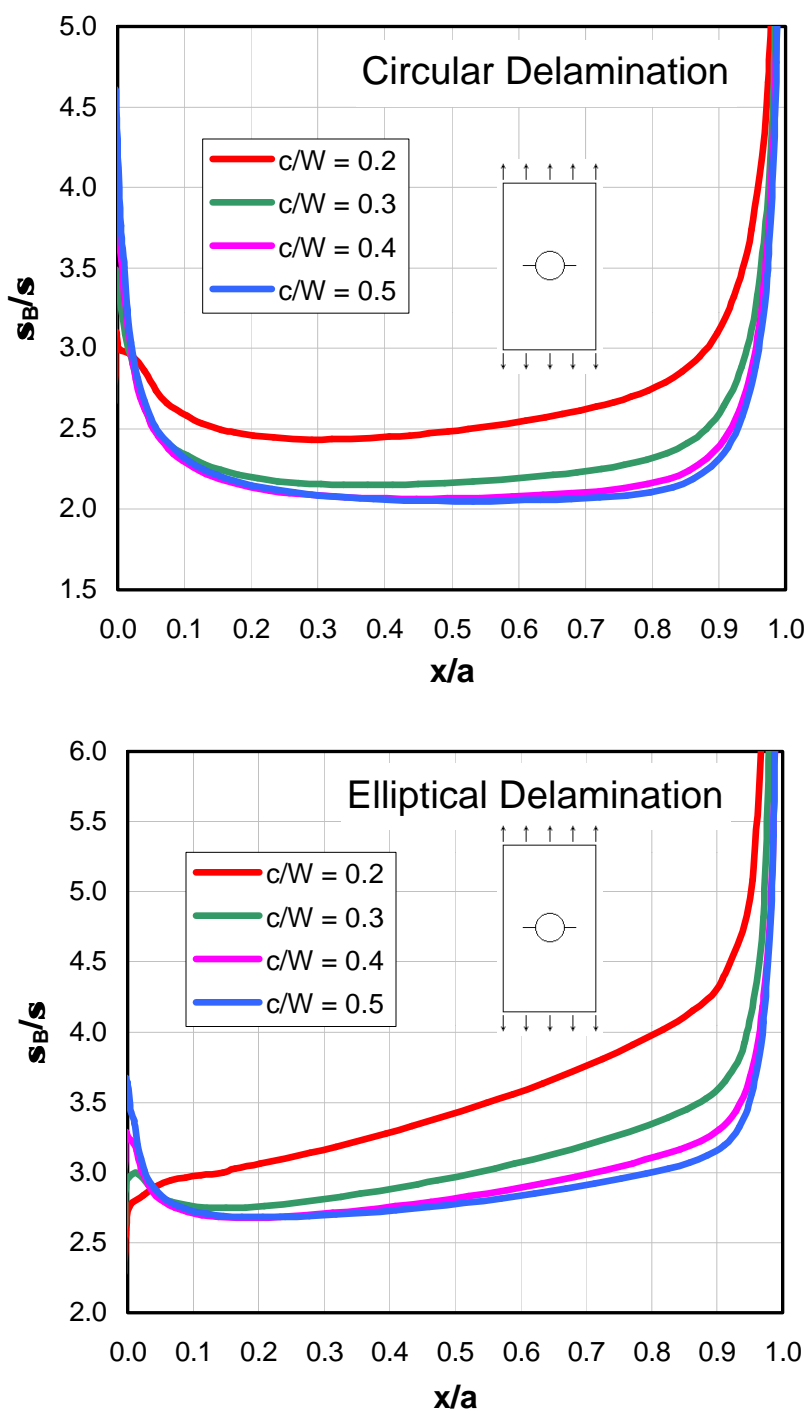

Figure 4.6: Variations of normalized bridging stress, $\sigma_{\mathrm{B}} / \sigma$, with relative distance from the crack-tip, $x / a \geq 0$, along the centreline of the aramid-epoxy layer, for the fully bridged central crack problem with circular and elliptical delaminations. (FEM analysis) 

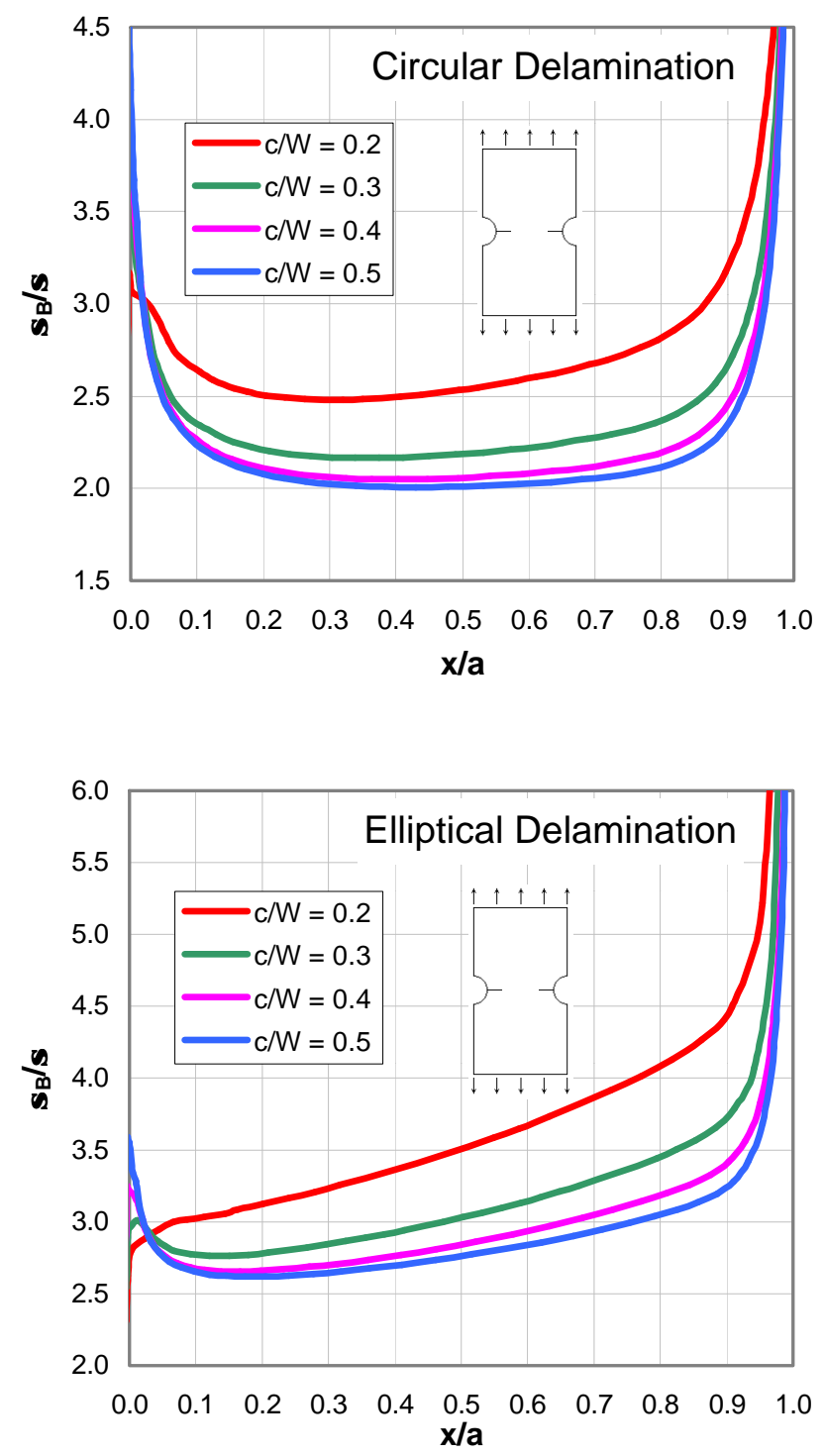

Figure 4.7: Variations of normalized bridging stress, $\sigma_{\mathrm{B}} / \sigma$, with relative distance from the crack-tip, $x / a \geq 0$, along the centreline of the aramid-epoxy layer, for the fully bridged double edge crack problem with circular and elliptical delaminations. (FEM analysis) 

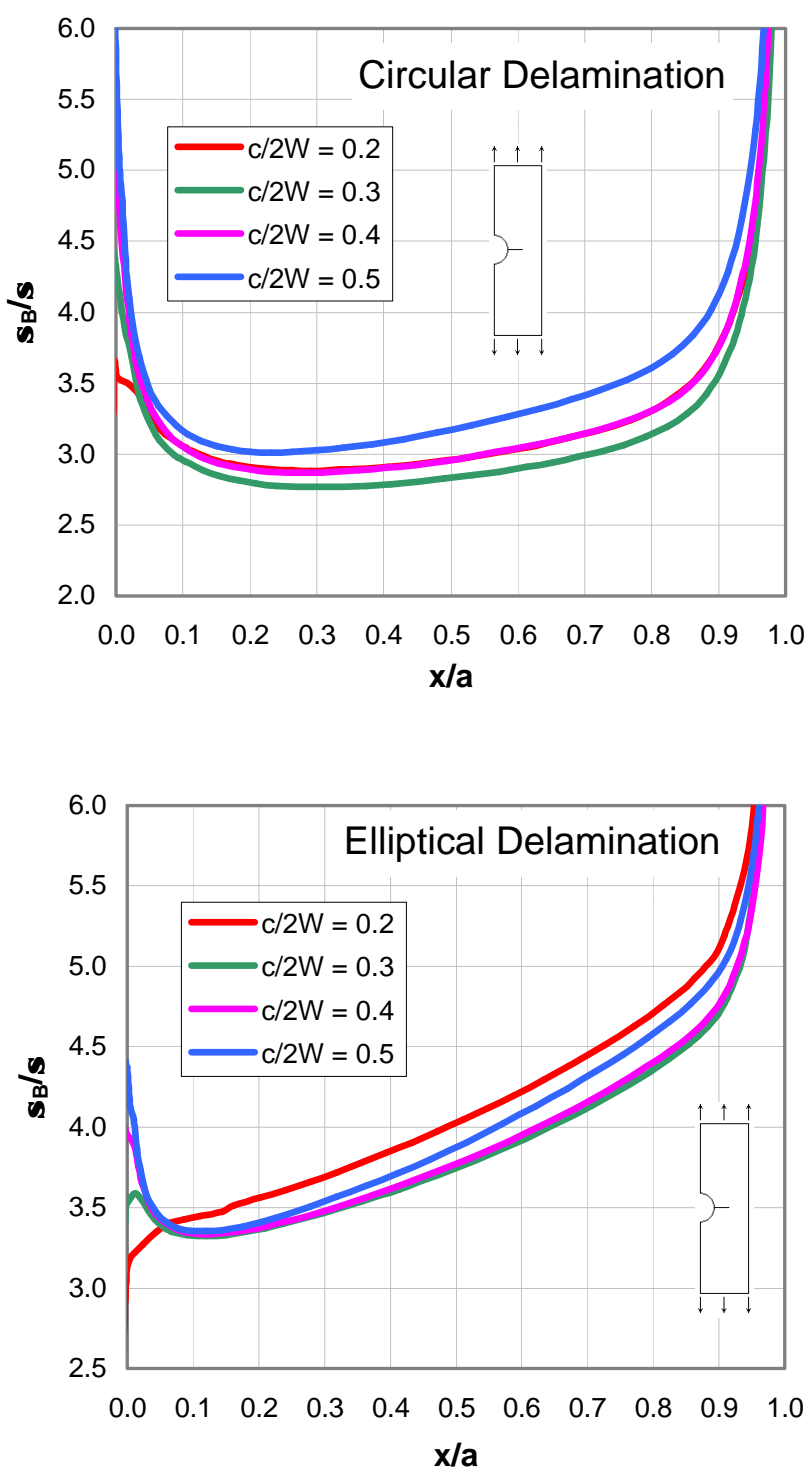

Figure 4.8: Variations of normalized bridging stress, $\sigma_{\mathrm{B}} / \sigma$, with relative distance from the crack-tip, $x / a \geq 0$, along the centreline of the aramid-epoxy layer, for the fully bridged edge crack problem with circular and elliptical delaminations. (FEM analysis) 
For the edge crack, double-edge crack, and central crack, the normalized bridging stress, $\sigma_{\mathrm{B}} / \sigma$, is higher for an elliptical delamination than for a circular delamination for all crack lengths, $c / W$ or $c / 2 W$. As a comparison, the central crack with $c / W=0.5$ and an elliptical delamination has a normalized bridging stress, $\sigma_{\mathrm{B}} / \sigma$, equal to 4.24 at $x / a=0$. For the same problem with a circular delamination, $\sigma_{\mathrm{B}} / \sigma$ $=3.64$ at $x / a=0$. The delamination zone shape and the bridging stress influence each other such that an increase in the delamination zone size decreases the bridging stress, and a decrease in the bridging stress increases the delamination zone size. As the delamination zone size increases, the amount of applied load transferred from the aluminum layers to the fibre layer decreases due to a smaller area for shear stress transfer between the metal and fibre layers. Thus, an increasing delamination causes a decrease in the stress in the fibres, and hence, a lower bridging stress, as shown in the obtained results.

The normalized bridging stress distribution, $\sigma_{\mathrm{B}} / \sigma$, for the central crack and the double-edge crack increases more rapidly for the elliptical delamination than for the circular delamination, with increasing distance away from the crack-tip, $x / a$. For the edge crack with an elliptical delamination, the normalized bridging stress, $\sigma_{\mathrm{B}} / \sigma$, for $c / 2 W=0.2$ is the highest compared to the rest of the crack lengths as mentioned previously. The normalized bridging stress increases more rapidly for the elliptical delamination than for the circular delamination, with distance away from the crack tip, $x$. Therefore, for a smaller delamination size, the stress in the fibres near the notch is greater and the influence of the stress concentration is more pronounced. The normalized bridging stress, $\sigma_{\mathrm{B}} / \sigma$, at $x / a=1.0$ for all geometries is generally larger for an elliptical delamination than for a circular delamination. As an example, a central crack with $c / W=0.2, \sigma_{\mathrm{B}} / \sigma=9.24$ for an elliptical delamination and only 7.85 for a circular delamination.

In the 3-D FEM analysis, the stress intensity factors, $K_{I}$, were calculated using the displacement extrapolation technique relating the stress intensity factor, $K_{I}$, to the half-crack vertical displacement, $v$, of the crack face by, Guinea, et al. (2000):

$$
K_{I}^{*}=\frac{H \sqrt{2 \pi}}{4}\left(\frac{v}{\sqrt{r}}\right)
$$

where, $\mathrm{r}=$ distance from the crack-tip, and

$$
\begin{aligned}
& H=\left\{\begin{array}{cl}
E & \text { plane stress } \\
\frac{E}{\left(1-v^{2}\right)} & \text { plane strain }
\end{array}\right. \\
& \mathrm{E}=\text { Young's Modulus } \\
& \mathrm{v}=\text { Poisson's Ratio }
\end{aligned}
$$

This was a well-established technique in FEM fracture mechanics analysis before the development of special formulas for the determination of stress intensity factors. The reason for using this is because, although MSC/Nastran v70.7 has an 
isotropic, 3-D crack-tip element, it contains a program bug which precludes the direct evaluation of these values when isotropic and anisotropic material properties are defined for different elements in the FE analysis.

\subsubsection{BEM Analysis}

\section{Bridging Stress Distribution}

In the analysis, the bridging stress at the crack mouth, $\sigma_{\mathrm{B}}{ }^{*}$ was again normalized with respect to the remote applied stress, $\sigma$ for the three physical problems considered. The computed normalized bridging stress, $\sigma_{\mathrm{B}} * / \sigma$, is plotted in Figures 4.9 to 4.11 as a function of the normalized crack length, $c / W$ or $c / 2 W$. These figures also show the variation of $\sigma_{\mathrm{B}} * / \sigma$ with the fibre-bridging power-law indices, $n$ and $m$ of 0 to 1.0 , and circular and elliptical delamination zone shapes.

For the central crack and the double-edge crack problems, the normalized bridging stress, $\sigma_{\mathrm{B}} * / \sigma$, decreases with increasing crack size, $c / W$, for both delamination shapes. For example, for a central crack with an elliptical delamination and $n=0.5$, at $c / W=0.2, \sigma_{\mathrm{B}} * / \sigma=4.55$, and at $c / W=0.5, \sigma_{\mathrm{B}} * / \sigma=3.40$. This decrease is attributed to the stress concentration near the hole where it has a larger influence on smaller crack lengths, and hence $\sigma_{\mathrm{B}} * / \sigma$ is highest for $c / W=0.2$, and decreases as $c / W$ increases. This same trend was observed in the results for the three dimensional FEM analysis for the bridging stress distributions.
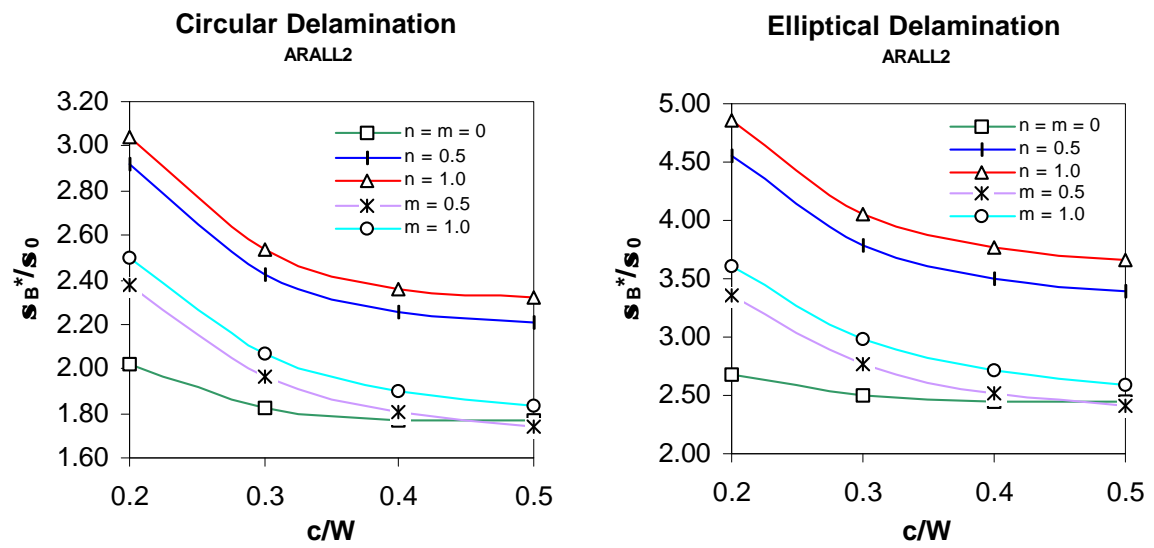

Figure 4.9: Variations of normalized bridging stress, $\sigma_{\mathrm{B}}{ }^{*} / \sigma$, with relative crack length, c/W, for the ARALL central crack problem with circular and elliptical delaminations. 

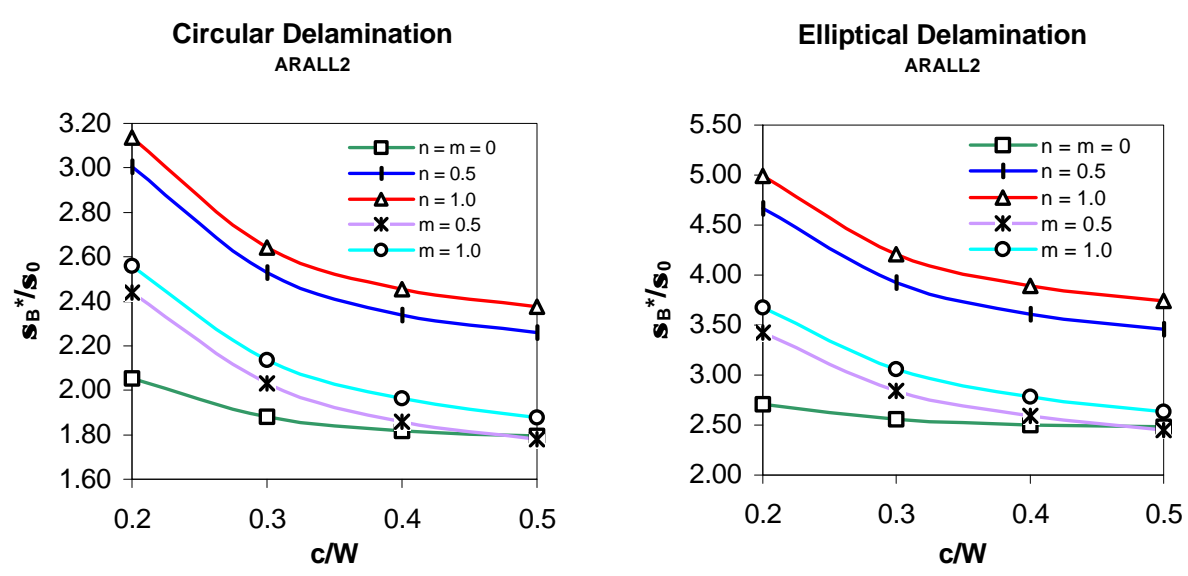

Figure 4.10: Variations of normalized bridging stress, $\sigma_{\mathrm{B}}{ }^{*} / \sigma$, with relative crack length, $\mathrm{c} / \mathrm{W}$, for the ARALL double edge crack problem with circular and elliptical delaminations.

The normalized bridging stress, $\sigma_{\mathrm{B}} * / \sigma$, for the central and double-edge cracks increases with increasing power-law index $n$ and $m$. For a double-edge crack with a circular delamination and $c / W=0.5, \sigma_{\mathrm{B}} * / \sigma$ for $n=0$ is 1.80 , while for $n=1.0$ it is equal to 2.38. The normalized bridging stress for $m=0.5$ and 1.0 is generally between the bridging stress for $n=0$ and 0.5 , for both delamination shapes and all crack lengths. The highest normalized bridging stress is for $n=1.0$, which represents a linear bridging stress distribution with decreasing stresses toward the crack-tip.

For an edge crack problem, the normalized bridging stress, $\sigma_{\mathrm{B}} * / \sigma$, decreases from $c / 2 W=0.2$ to $c / 2 W=0.3$ and then increases as the crack length increases from $c / 2 W=0.3$ to $c / 2 W=0.5$. This trend is again the same as the one predicted by the three dimensional FEM analysis for the normalized bridging stress distributions. As an example, for an elliptical delamination and $n=0.5$, the normalized bridging stress, $\sigma_{\mathrm{B}} * / \sigma$ is 5.43 at $c / 2 W=0.2$ and decreases to 5.09 at $c / 2 W=0.3$ and then increases to 5.25 and 5.56 at $c / 2 W=0.4$ and 0.5 , respectively.

The normalized bridging stress, $\sigma_{\mathrm{B}} * / \sigma$, for the edge crack increases as the power-law index, $m$ and $n$ increases, for both delamination shapes. The highest normalized bridging stress for the edge crack is also represented by the index $n=1.0$. It should be noted that for the edge crack a constant bridging stress represented by $n=m=$ 0 does not show a decrease around $c / 2 W=0.3$ but only increases from $c / W=0.2$ to 0.5 . 

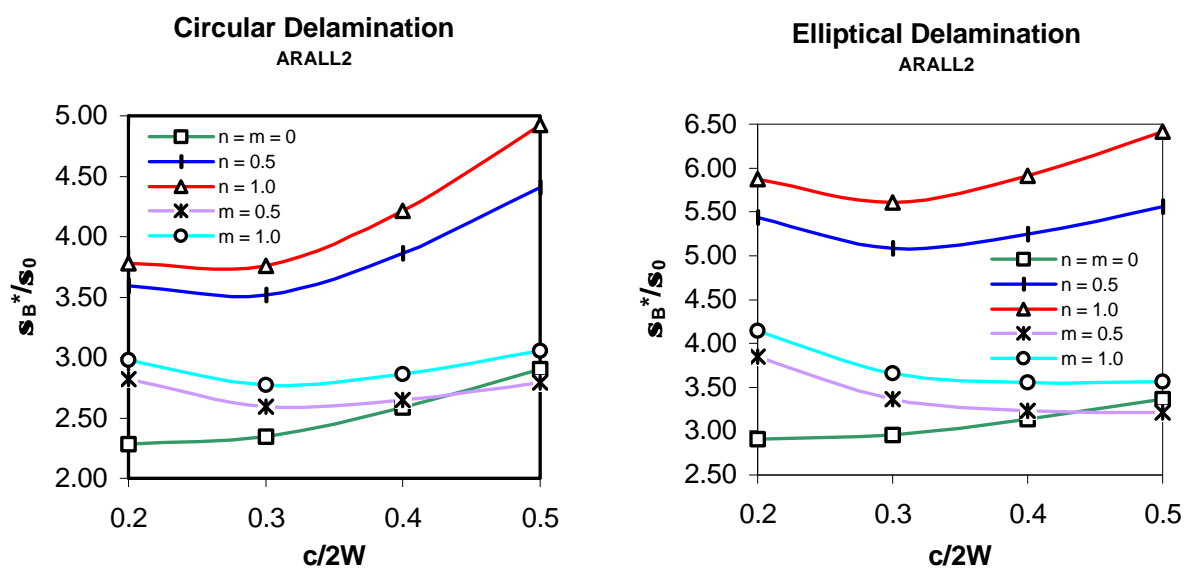

Figure 4.11: Variations of normalized bridging stress, $\sigma_{\mathrm{B}}{ }^{*} / \sigma$, with relative crack length, $\mathrm{c} / 2 \mathrm{~W}$, for the ARALL edge crack problem with circular and elliptical delaminations.

For all geometries, the delamination zone shape influences the bridging stresses such that the normalized bridging stress, $\sigma_{\mathrm{B}} * / \sigma$, is higher for an elliptical delamination as compared to a circular delamination. This is the same trend as seen in the FEM analysis in the previous section. Comparing an edge crack with $c / 2 W=0.2$ and $n=1.0, \sigma_{\mathrm{B}} * / \sigma=5.872$ for an elliptical delamination, while for a circular delamination, $\sigma_{\mathrm{B}} * / \sigma=3.776$.

In general, the 3-D FEM results lie between a constant distribution given by $n$ $=0$, and a linear distribution given by $n=1.0$. The 2-D BEM results also lie between these two bounds of power-law index, being closer to $n=0.5$. The bridging stresses generally decrease toward the crack-tip and it can be concluded that the profiles are that of a 'strain-hardening' material. Therefore, the power-law index range of $0 \leq n \leq 1.0$, as obtained by the 2-D BEM analysis and confirmed by the 3-D FEM analysis, describes the variation of bridging stresses in fibre-metal laminates with notches. Now, it remains to be seen if the results for the stress intensity factors are in agreement with this determined power-law index range.

\section{Stress Intensity Factors}

The mode I stress intensity factor, $K_{I}$, was calculated using the traction formula, equation 3.34, in the two- dimensional boundary element method (BEM) analysis. This stress intensity factor was normalized by $\sigma \sqrt{\pi c}$, and plotted with 
respect to the normalized crack length, $c / W$ or $c / 2 W$ in Figures 4.12 to 4.14 . These figures show the normalized stress intensity factors, $K_{I} / \sigma \sqrt{\pi c}$, for the edge crack, double-edge crack, and central crack with circular and elliptical delamination zone shapes and power-law indices, $n$ and $m$, equal to $0,0.5$, and 1.0.

The normalized stress intensity factor, $K_{I} / \sigma \sqrt{\pi c}$, increases gradually with increasing crack length, $c / W$ for the central crack and the double-edge crack geometries for both delamination shapes and power-law indices, $n$ and $m$, equal to 0.5 and 1.0. The normalized stress intensity factor slightly decreases from $c / W=0.2$ and then increases up to $c / W=0.5$ for the assumed constant bridging stress distribution represented by $n=$ $m=0$. The normalized stress intensity factor, $K_{I} / \sigma \sqrt{\pi c}$, also increases with increasing power-law index $n$, for both the circular and elliptical delamination shapes. For both delamination shapes, the stress intensity factors for the power-law index $m=$ 0.5 and 1.0 are almost the same in magnitude and significantly lower than those for the other power-law indices.
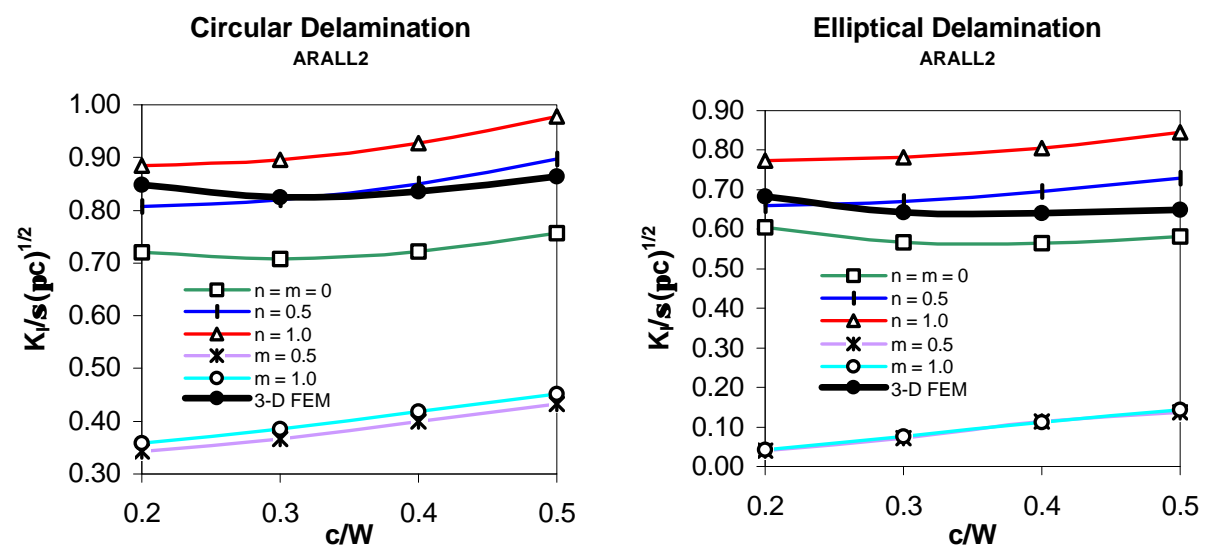

Figure 4.12: Variations of normalized crack-tip stress intensity factor, $\mathrm{K}_{\mathrm{I}} / \sigma(\pi \mathrm{c})^{1 / 2}$, with relative crack length, $\mathrm{c} / \mathrm{W}$, for the ARALL central crack problem with circular and elliptical delaminations. 

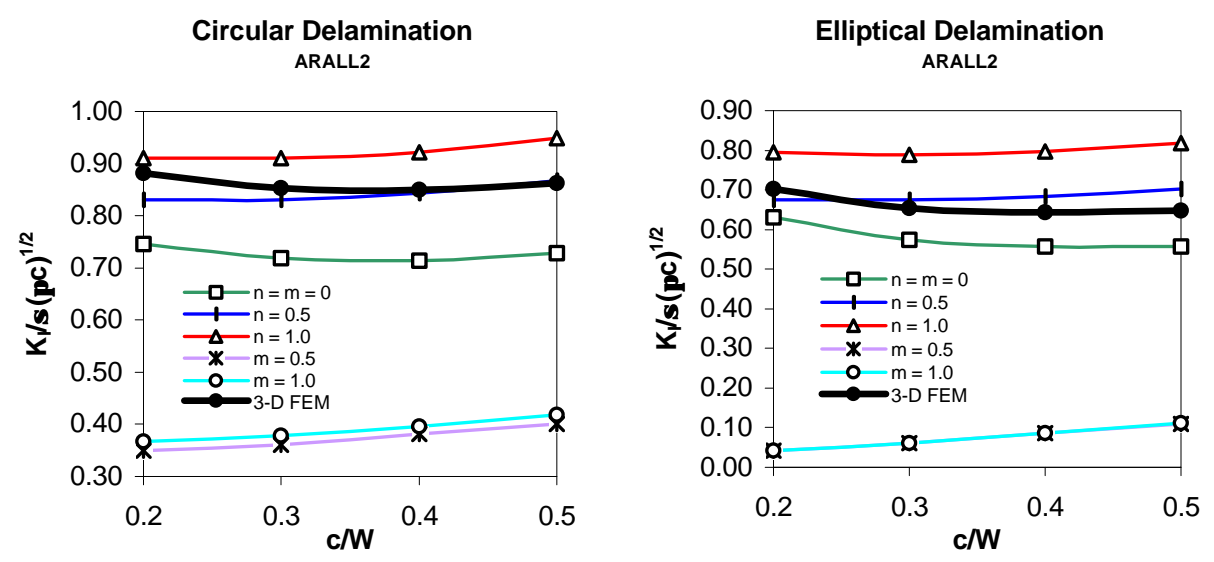

Figure 4.13: Variations of normalized crack-tip stress intensity factor, $K_{I} / \sigma(\pi c)^{1 / 2}$, with relative crack length, $\mathrm{c} / \mathrm{W}$, for the ARALL double edge crack problem with circular and elliptical delaminations.

The normalized stress intensity factor, $K_{I} / \sigma \sqrt{\pi c}$, for the edge crack, also increases as the crack length, $c / 2 W$ increases. This increase is more rapid than that for the central and double-edge cracks. The normalized stress intensity factors also increase as the power-law index $n$ increases, with the exception of an elliptical delamination shape at crack sizes of $c / 2 W>0.3$, where the constant distribution represented by $n=m=0$ is higher than the one for $n=0.5$. In the case of an elliptical delamination shape, the magnitude of the normalized stress intensity factor obtained using $m=0.5$ and 1.0 is almost zero for all crack lengths. Also, the magnitude of the normalized stress intensity factor is significantly smaller for $m=0.5$ and 1.0 as compared to that for the power law index $n$.

The BEM results in Figures 4.12 to 4.14 show that the delamination zone shape has an influence on the stress intensity factor for the crack in the metal layer. A smaller delamination zone size results in lower crack-tip stress intensity factors. This can be seen, for example, in the edge crack problem with $n=0.5$ and $c / W=0.5$ where $K_{I} / \sigma \sqrt{\pi c}=0.858$ for an elliptical delamination and $K_{I} / \sigma \sqrt{\pi c}=1.265$ for a circular delamination. 

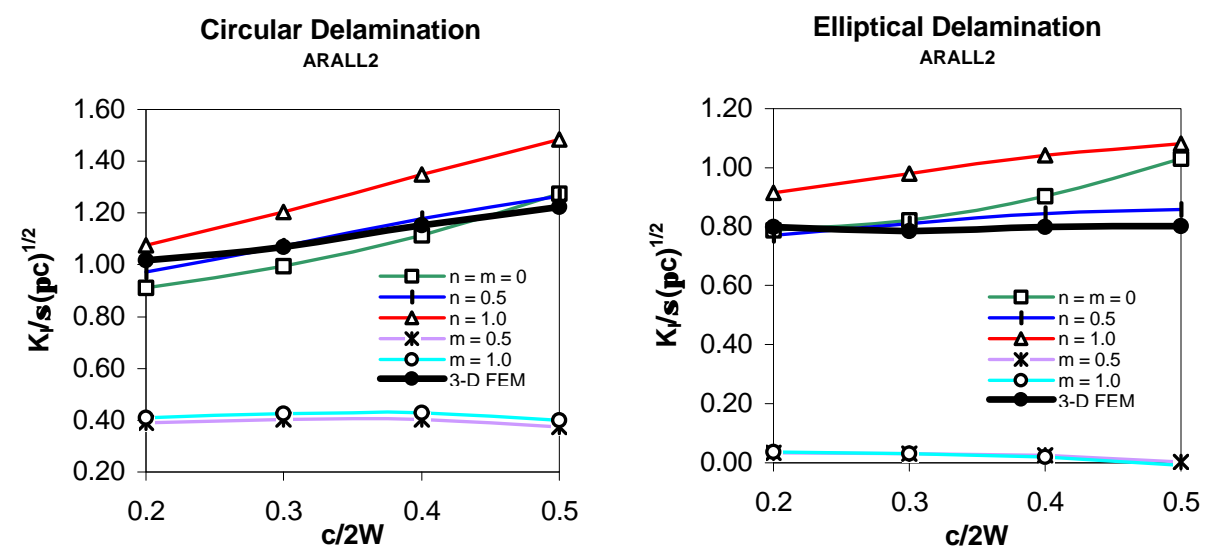

Figure 4.14: Variations of normalized crack-tip stress intensity factor, $K_{I} / \sigma(\pi c)^{1 / 2}$, with relative crack length, $\mathrm{c} / \mathbf{2 W}$, for the ARALL edge crack problem with circular and elliptical delaminations.

The 2-D BEM results suggest that the bridging stress distribution represented by $m=0.5$ and $m=1.0$ is not applicable in the two dimensional analysis because it cannot predict accurate values of stress intensity factor. A possible explanation for this is that the stresses in the fibres are higher with decreasing distance from the hole due to the stress concentration there, but the $m$ index assumes a decreasing stress profile towards the hole. As a result, the stress intensity factors are very small for the powerlaw index, $m>0$.

The normalized stress intensity factors from a three-dimensional FEM analysis are plotted with the two-dimensional BEM stress intensity factors from FEM and lie between the power law index $n=0$ and $n=1.0$ for all crack geometries and delamination shapes. The FEM results are generally closer to $n=0.5$ and thus this index best describes the variation of stress intensity factors with crack length, $c / W$ or $c / 2 W$. The percentage differences between the FEM computed normalized stress intensity factors, $K_{I} / \sigma(\pi \mathrm{c})^{1 / 2}$, and those obtained from the 2-D BEM analysis with $n=0.5$ are listed in Table 4.2 for all the geometric cases treated. With some exceptions, the percentage deviations were generally less than about 6 percent; the maximum deviation was $12.3 \%$. This finding is consistent with the findings of Cain and Tan (1997). From the results obtained, it was also found that the uniform bridging stress distribution $(n=$ 0) proposed by Marissen (1988), for example, can lead to a 35\% underestimation of the stress intensity factor for the edge crack problem with an elliptical delamination and about $22 \%$ for the corresponding circular delamination. 
Table 4.2: Computed normalized stress intensity factors for the FEM analysis and the BEM analysis with $\mathbf{n}=0.5$.

\begin{tabular}{|c|c|c|c|c|c|c|c|}
\hline \multirow{2}{*}{\multicolumn{2}{|c|}{$n \equiv 0.5$}} & \multicolumn{3}{|c|}{ Elliptical Delamination } & \multicolumn{3}{|c|}{ Circular Delamination } \\
\hline & & \multirow{2}{*}{$\begin{array}{c}\text { BEM } \\
\mathrm{K}_{\mathrm{l}} / \sigma(\pi \mathrm{c})^{1 / 2}\end{array}$} & \multirow{2}{*}{$\begin{array}{c}\text { FEM } \\
\mathrm{K}_{\mathrm{l}} / \sigma(\pi \mathrm{c})^{1 / 2}\end{array}$} & \multirow{2}{*}{ \%diff } & \multirow{2}{*}{$\begin{array}{c}\text { BEM } \\
\mathrm{K}_{\mathrm{l}} / \sigma(\pi \mathbf{c})^{1 / 2}\end{array}$} & \multirow{2}{*}{$\begin{array}{c}\text { FEM } \\
\mathrm{K}_{\mathrm{l}} / \sigma(\pi \mathrm{c})^{1 / 2}\end{array}$} & \multirow{2}{*}{$\%$ dif } \\
\hline Geometry & $\mathbf{c} / \mathbf{W}$ & & & & & & \\
\hline \multirow{4}{*}{ Central } & 0.2 & 0.660 & 0.683 & 3.4 & 0.807 & 0.848 & 4.8 \\
\hline & 0.3 & 0.670 & 0.642 & -4.4 & 0.820 & 0.824 & 0.5 \\
\hline & 0.4 & 0.695 & 0.640 & -8.6 & 0.850 & 0.836 & -1.7 \\
\hline & 0.5 & 0.730 & 0.650 & -12.3 & 0.897 & 0.864 & -3.8 \\
\hline \multirow{4}{*}{ Double Edge } & 0.2 & 0.676 & 0.703 & 3.8 & 0.830 & 0.881 & 5.8 \\
\hline & 0.3 & 0.674 & 0.655 & -2.9 & 0.831 & 0.853 & 2.6 \\
\hline & 0.4 & 0.684 & 0.644 & -6.2 & 0.843 & 0.850 & 0.8 \\
\hline & 0.5 & 0.703 & 0.647 & -8.7 & 0.868 & 0.862 & -0.7 \\
\hline \multirow{4}{*}{ Edge } & 0.2 & 0.770 & 0.800 & 3.8 & 0.971 & 1.017 & 4.5 \\
\hline & 0.3 & 0.811 & 0.785 & -3.3 & 1.072 & 1.069 & -0.3 \\
\hline & 0.4 & 0.845 & 0.798 & -5.9 & 1.178 & 1.154 & -2.1 \\
\hline & 0.5 & 0.858 & 0.802 & -7.0 & 1.265 & 1.223 & -3.4 \\
\hline
\end{tabular}

The stress intensity factors obtained by the FEM analysis therefore confirm the range of those obtained by the two-dimensional BEM analysis. Therefore, it can be concluded that a three-dimensional fibre-metal laminate can be modelled using a twodimensional BEM analysis yielding a fibre-bridging index range which adequately describes the variations and magnitudes of the bridging stresses and stress intensity factors. The two-dimensional analysis is significantly less time consuming and less labour intensive than the corresponding three-dimensional analysis.

\subsection{Partial Bridging of Notched Fibre-Metal Laminates}

It was shown in the previous Section for the full bridging analysis of cracks emanating from a hole, that the stresses in bridging fibres near the hole are very high. This could lead to fibre failure in that region and result in partial bridging of the crack. It is therefore of interest to obtain the bridging stresses and stress intensity factors for such situations and compare these values with the full bridging case.

Two cases of partial bridging were analysed in this study. The first case was that of $75 \%$ of the crack length being bridged by fibres, and the second case was that of $50 \%$ of the crack length being bridged by fibres. This implies that $25 \%$ and $50 \%$ of the 
fibres have failed in the first and second cases, respectively. Also, an unbridged laminate was analysed by FEM and BEM to quantitatively determine the benefit of fibre bridging in reducing the crack-tip stress intensity factors.

The same three rectangular plates considered in Section 4 were analysed here. The loading conditions and geometry were the same as defined in Section 4.1 and shown in Figure 4.1. The bridging length, $b$, was varied depending on the amount of bridging present. For the $75 \%$ bridging case, $b$ was set equal to $0.75 a$, and for $50 \%$ bridging $b$ was equal to $0.5 a$. The mesh in the two-dimensional BIE analysis was the same as the one used for the full bridging case for a given cracked plate, with the elements in the $25 \%$ and $50 \%$ un-bridged regions of the crack now being traction free.

As before, two delamination shapes, quarter-circular and quarter-elliptical with a major-minor axis ratio of 2 , were used in the partial bridging analysis. This assumes that the delamination shape present in full bridging will remain the same for partial bridging. The delamination zone size, $d$, was taken at the last intact fibre away from the crack-tip in the bridged zone. For the $75 \%$ and $50 \%$ bridging cases, $d$ was determined from an equation of a circle for the circular delamination, and an equation of an ellipse with a 2-1 major-minor axis ratio for the elliptical delamination.

The meshes used in the three-dimensional FE analysis of partial bridging were the same as those used for full bridging in Section 4. The nodes of the elements in the unbridged region in the fibre layer were unrestrained to represent the failed fibres. The same fibre-metal laminate material as before, namely, ARALL 2-2/1, was considered in the partial bridging analysis.

\subsection{Results and Discussion}

The bridging stress and the stress intensity factor results for an edge crack, double edge crack, and central crack with $75 \%$ and $50 \%$ partial bridging are presented in this section. The results of an unbridged fibre-metal laminate for all the cracked geometries are also presented. A comparison of the bridging stresses and stress intensity factors is made between the case of full bridging and that of partial bridging or no bridging at all. The bridging stress and stress intensity factor results obtained by a BEM analysis are also compared to those obtained from the 3-D FEM analysis. 


\subsubsection{FEM Analysis}

\section{Bridging Stress Distribution}

The bridging stress, $\sigma_{\mathrm{B}}$, was taken along the centreline of the fibre layer and normalized with respect to the remote applied stress, $\sigma$. The variations of normalized bridging stress, $\sigma_{\mathrm{B}} / \sigma$, with relative distance, $x / a$, from the crack tip are plotted in Figures 5.1 to 5.6. These figures show the results for a central, double edge, and edge crack with $75 \%$ and $50 \%$ partial bridging zones, and relative normalized crack lengths, $c / W$ or $c / 2 W$. The material properties for the results presented correspond to those of ARALL. The normalized bridging stress, $\sigma_{\mathrm{B}} / \sigma$, for all crack geometries increases very rapidly near $x / a=0.75$ and $x / a=0.50$ for the $75 \%$ and $50 \%$ partial bridging cases, respectively. This is due to the singularity at that location as a result of a crack now present in the fibre layer. The normalized bridging stress also increases near the crack tip, corresponding to $x / a=0$. As discussed in Section 4 , this is likely due to the enforced singularity of the quarter-point crack-tip elements in the aluminum layer adjacent to the mid-point elements in the fibre-reinforced layer in the numerical model.

In the $75 \%$ and $50 \%$ partial bridging cases, the normalized bridging stress for the central crack and double edge crack decreases as the relative crack length, $c / W$ increases. For the edge crack, the normalized bridging stress increases as $c / 2 \mathrm{~W}$ increases. These trends show that the influence of the stress concentration on the bridging stresses is the same as discussed in the previous chapter for the case of full bridging. More specifically, the stress concentration near the notch increases the bridging stresses along the crack and the effect is more pronounced with shorter crack lengths. The trends for the edge crack problem differ than those for the other geometries due to the bending effect arising from its geometry. The bending effect is the dominating factor for larger crack lengths. As a result, for the edge crack, the bridging stresses increase with increasing crack length.

For both partial bridging cases studied here, the normalized bridging stress is higher for the elliptical delamination than for the circular delamination. Again, this trend is the same as that seen in fully bridged laminates in the previous chapter and this is due to a greater amount of stress transferred from the metal layers to the fibre layer for a smaller delamination size. 

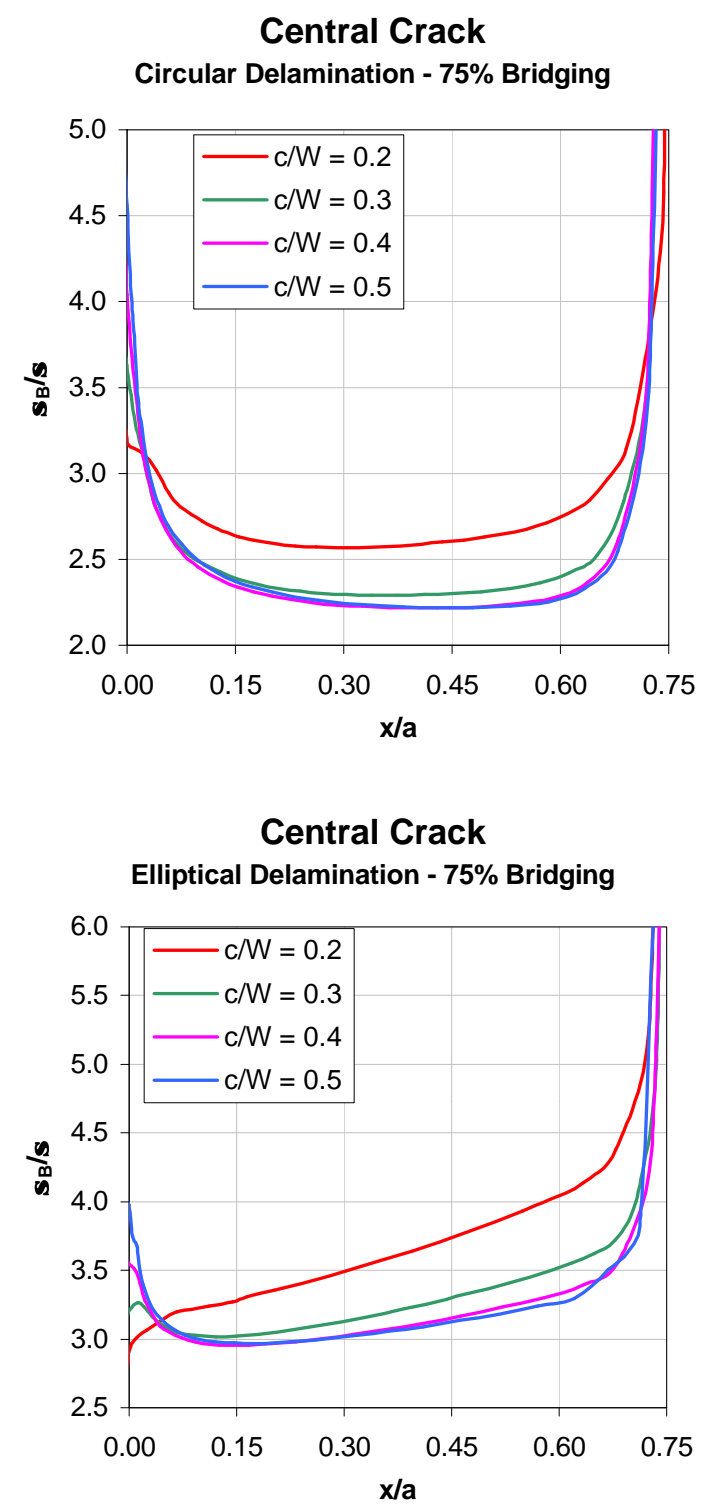

Figure 5.1: Variations of normalized bridging stress, $\sigma_{\mathrm{B}} / \sigma$, with relative distance from the crack tip, $x / a \geq 0$, along the centreline of the aramid-epoxy layer, for the $75 \%$ partially bridged central crack problem with circular and elliptical delaminations. (FEM Analysis) 

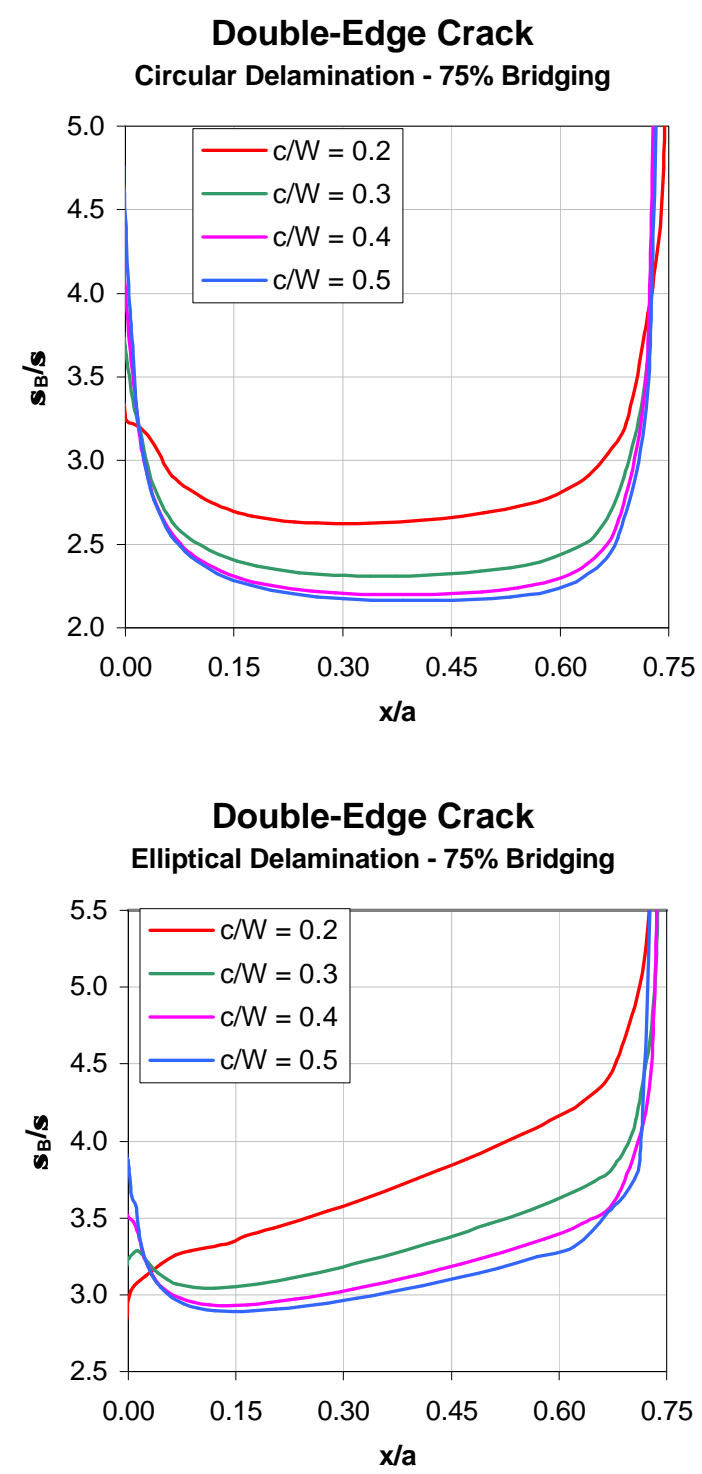

Figure 5.2: Variations of normalized bridging stress, $\sigma_{\mathrm{B}} / \sigma$, with relative distance from the crack tip, $x / a \geq 0$, along the centreline of the aramid-epoxy layer, for the $75 \%$ partially bridged double edge crack problem with circular and elliptical delaminations. (FEM Analysis) 

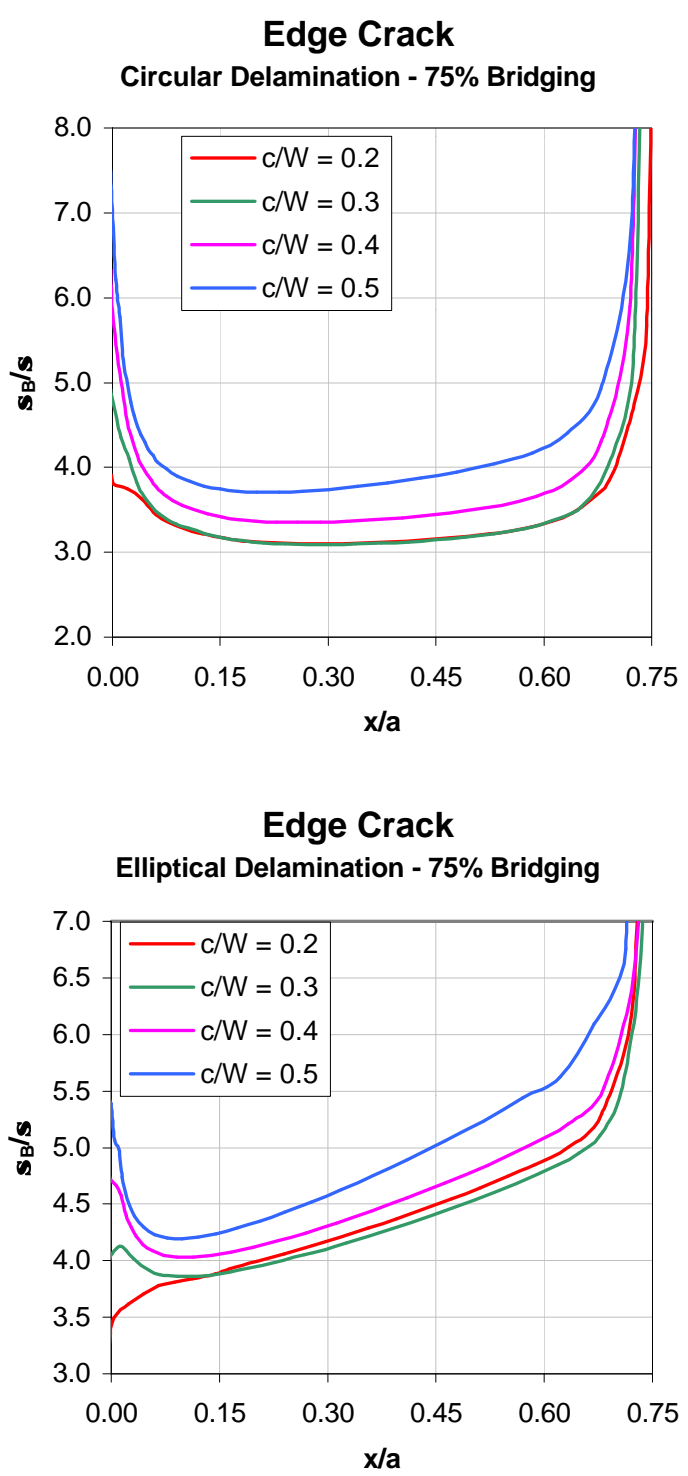

Figure 5.3: Variations of normalized bridging stress, $\sigma_{\mathrm{B}} / \sigma$, with relative distance from the crack tip, $x / a \geq 0$, along the centreline of the aramid-epoxy layer, for the $75 \%$ partially bridged edge crack problem with circular and elliptical delaminations. (FEM Analysis) 


\section{Central Crack}

Circular Delamination - 50\% Bridging

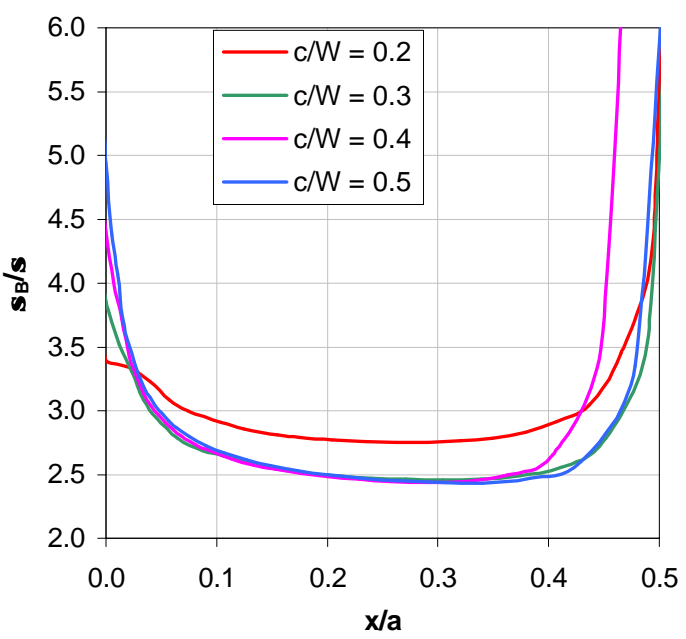

\section{Central Crack}

Elliptical Delamination - 50\% Bridging

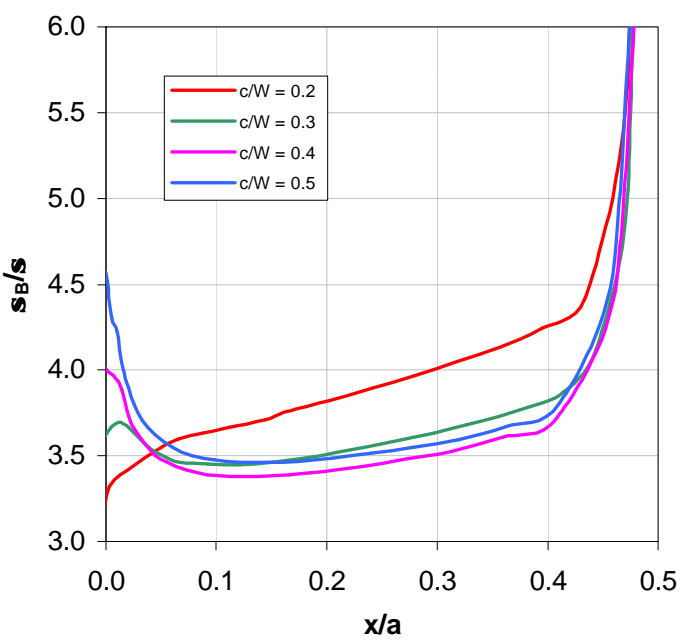

Figure 5.4: Variations of normalized bridging stress, $\sigma_{\mathrm{B}} / \sigma$, with relative distance from the crack tip, $x / a \geq 0$, along the centreline of the aramid-epoxy layer, for the $50 \%$ partially bridged central crack problem with circular and elliptical delaminations. (FEM Analysis) 

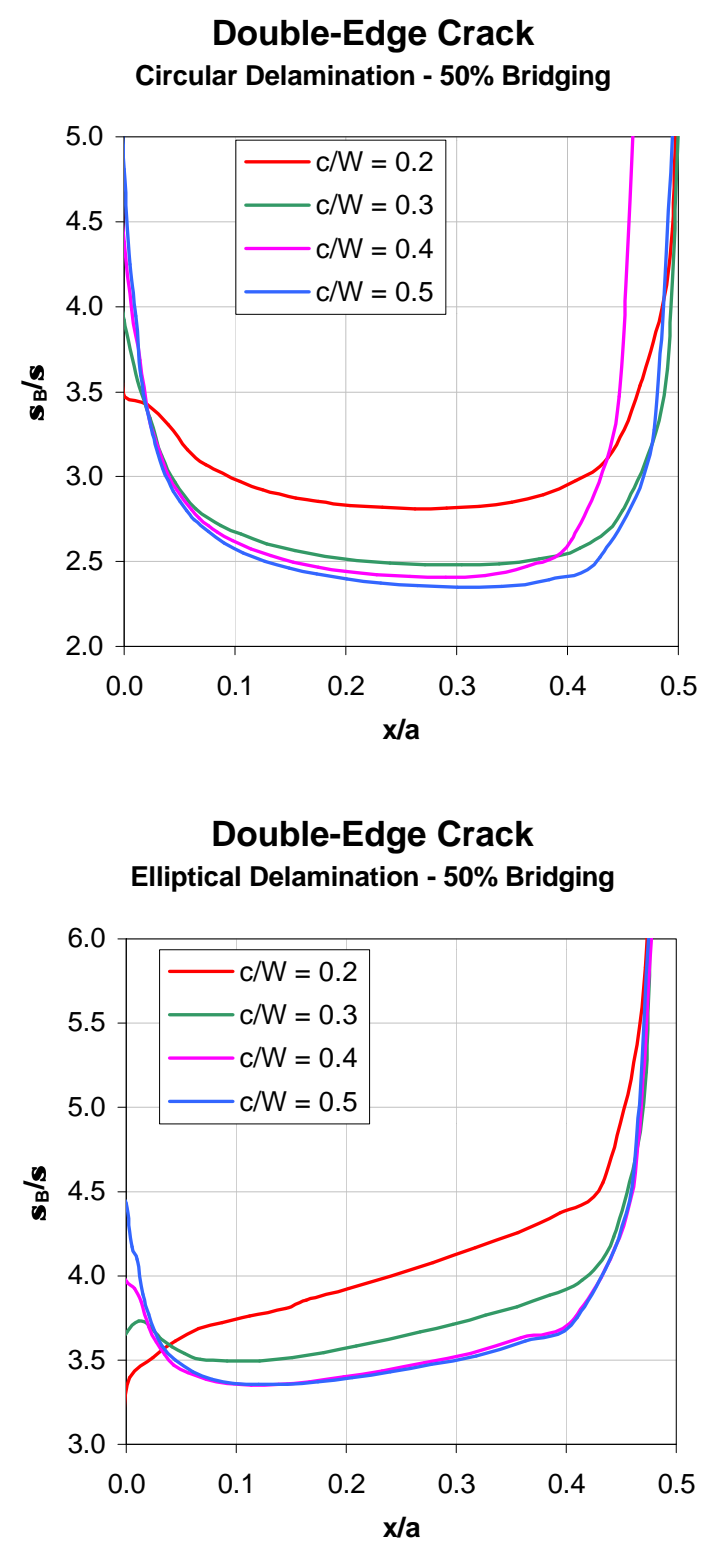

Figure 5.5: Variations of normalized bridging stress, $\sigma_{\mathrm{B}} / \sigma$, with relative distance from the crack tip, $x / a \geq 0$, along the centreline of the aramid-epoxy layer, for the $50 \%$ partially bridged double edge crack problem with circular and elliptical delaminations. (FEM Analysis) 


\section{Edge Crack}

Circular Delamination - 50\% Bridging

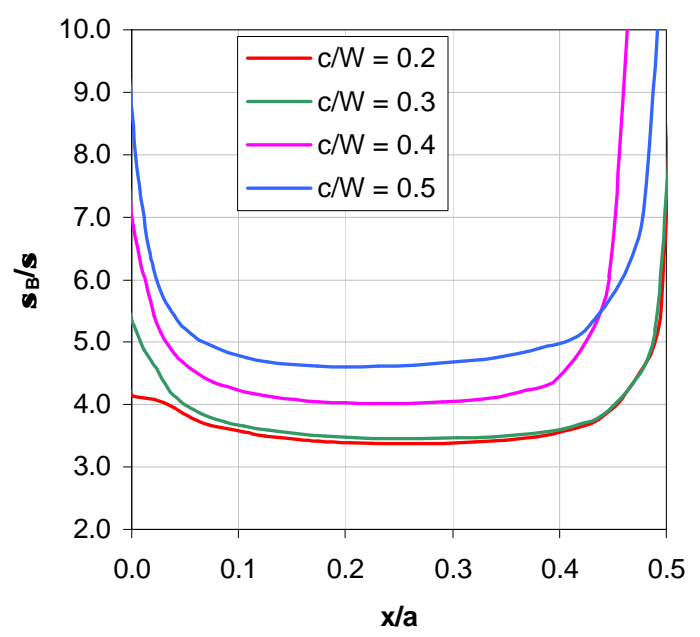

\section{Edge Crack}

Elliptical Delamination - 50\% Bridging

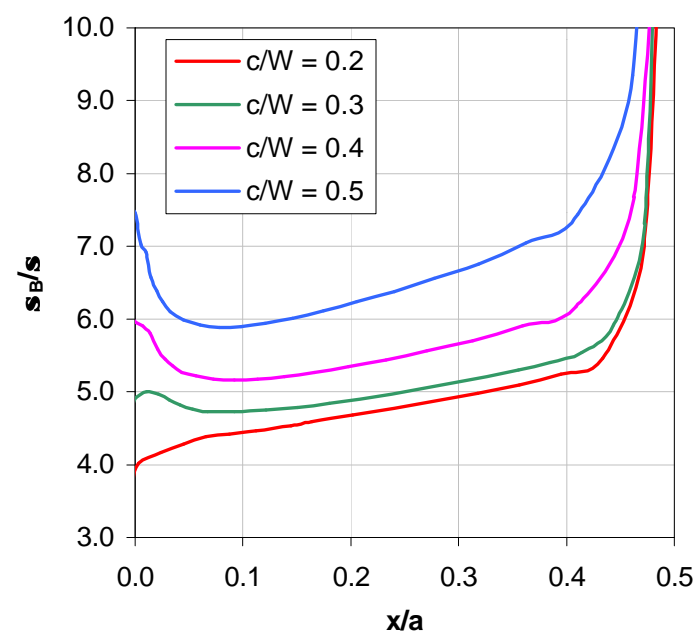

Figure 5.6: Variations of normalized bridging stress, $\sigma_{\mathrm{B}} / \sigma$, with relative distance from the crack tip, $x / a \geq 0$, along the centreline of the aramid-epoxy layer, for the $50 \%$ partially bridged edge crack problem with circular and elliptical delaminations. (FEM Analysis) 
The variations of percentage difference between partial bridging and full bridging is shown in Figures 5.7 to 5.12. These figures show that the normalized bridging stress increases as the relative bridging zone length, $b$ decreases. As an example, for the edge crack at $x / a=0$, with an elliptical delamination and $c / 2 W=0.5$, $\sigma_{\mathrm{B}} / \sigma=4.37$ for full bridging, $\sigma_{\mathrm{B}} / \sigma=5.39$ for $75 \%$ bridging, and $\sigma_{\mathrm{B}} / \sigma=7.46$ for $50 \%$ bridging.

For the central crack and double edge crack with $75 \%$ partial bridging, the differences of the bridging stress between the full bridging case are approximately in the range of $5 \%$ to $7 \%$, and $7 \%$ to $13 \%$ for the circular delamination and elliptical delamination, respectively. For the $50 \%$ partial bridging, the differences between the full bridging case increase to about $11 \%$ to $14 \%$, and $17 \%$ to $26 \%$ for the circular delamination and elliptical delamination, respectively. The bridging stresses for the edge crack problem with $75 \%$ bridging has a percentage difference from the full bridging case of approximately $7 \%$ to $20 \%$ for circular delamination, and $10 \%$ to $25 \%$ for elliptical delamination. At $50 \%$ bridging, the corresponding ranges of differences increase to $15 \%$ to $35 \%$ and $20 \%$ to $50 \%$. It can be seen from these results that a decrease in the amount of fibres bridging the crack increases the bridging stress in the remaining fibres. With less fibres bridging the crack, the remaining fibres will have higher bridging stresses for an equal magnitude of applied stress.

\section{Stress Intensity Factors}

The percentage difference of $K_{I} / \sigma \sqrt{\pi c}$ between the full bridged and each partial bridging case of the different crack configurations are shown in Tables 5.1 to 5.3. These results show that the normalized stress intensity factor increases as the bridging length is decreased as to be expected. A decrease in the amount of fibres bridging the crack causes the stress intensity factor in the aluminum layer to increase because the crack is able to open up more for a decreased bridging length. 

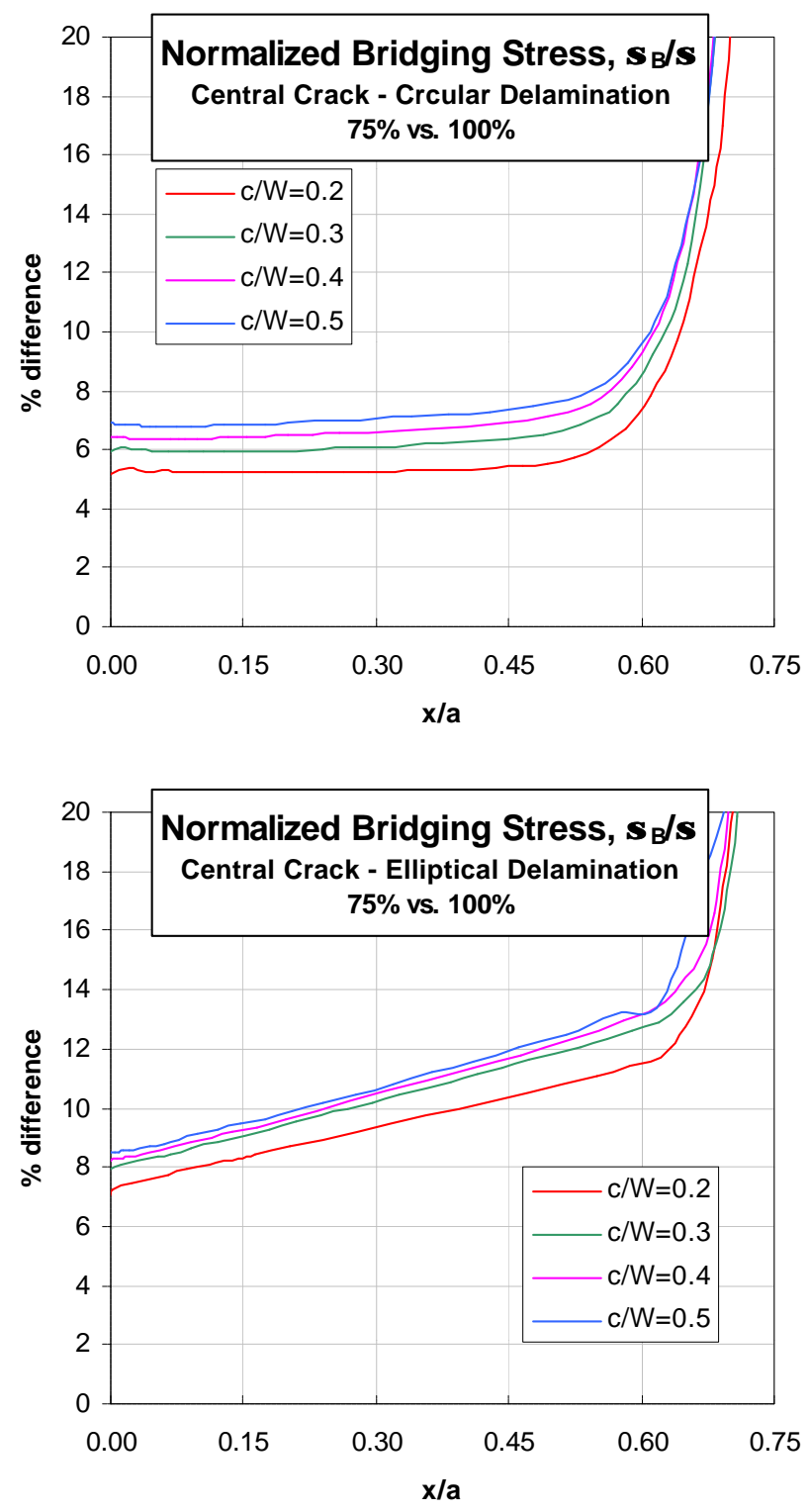

Figure 5.7: Percentage difference between $75 \%$ and $100 \%$ bridging, for the variations of the normalized bridging stress, $\sigma_{\mathrm{B}} / \sigma$, with relative distance from the crack-tip, $x / a$, along the centreline of the aramidepoxy layer. Central crack problem with circular and elliptical delaminations. 

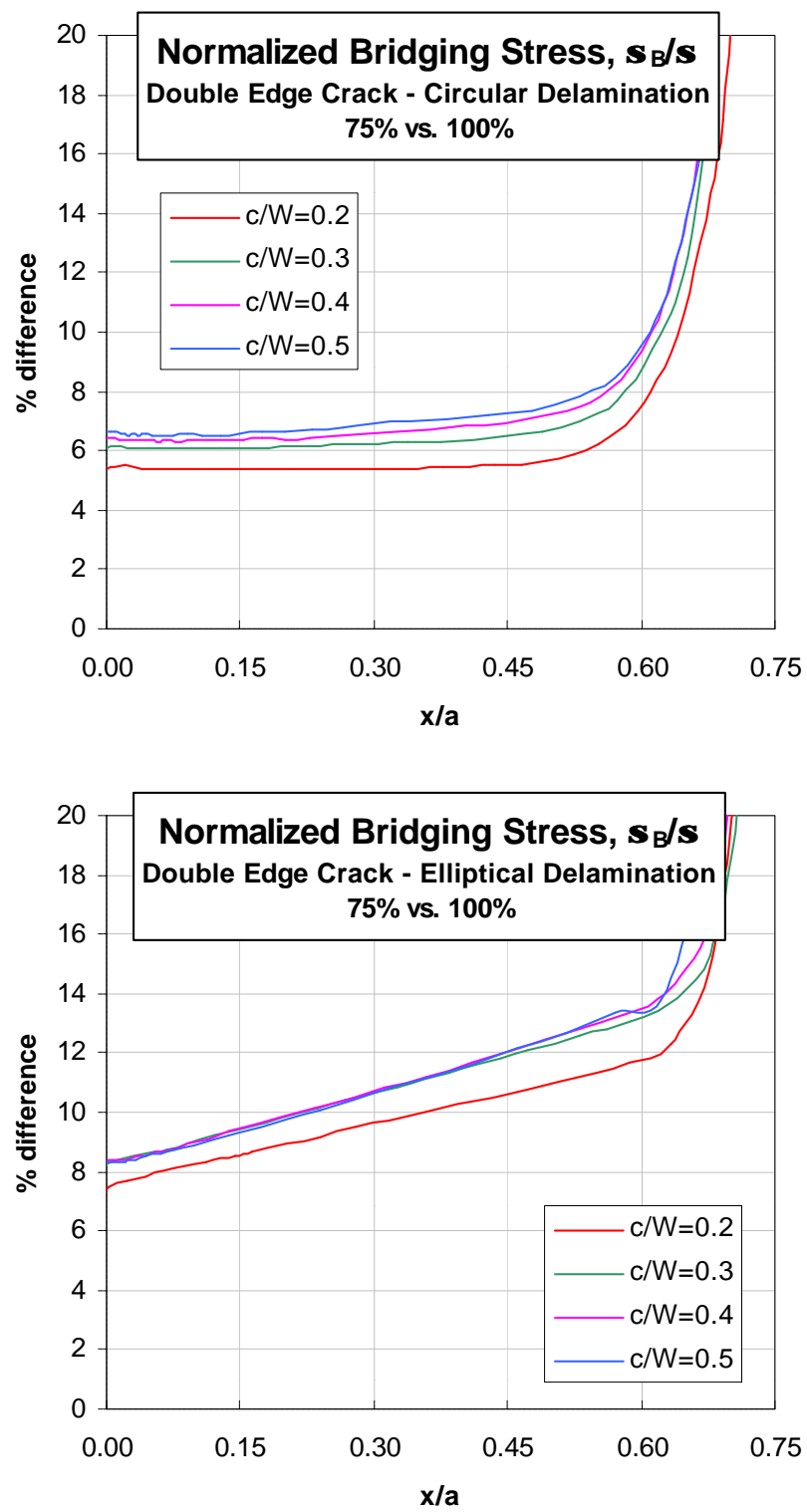

Figure 5.8: Percentage difference between $75 \%$ and $100 \%$ bridging, for the variations of the normalized bridging stress, $\sigma_{\mathrm{B}} / \sigma$, with relative distance from the crack-tip, $x / a$, along the centreline of the aramidepoxy layer. Double Edge crack problem with circular and elliptical delaminations. 

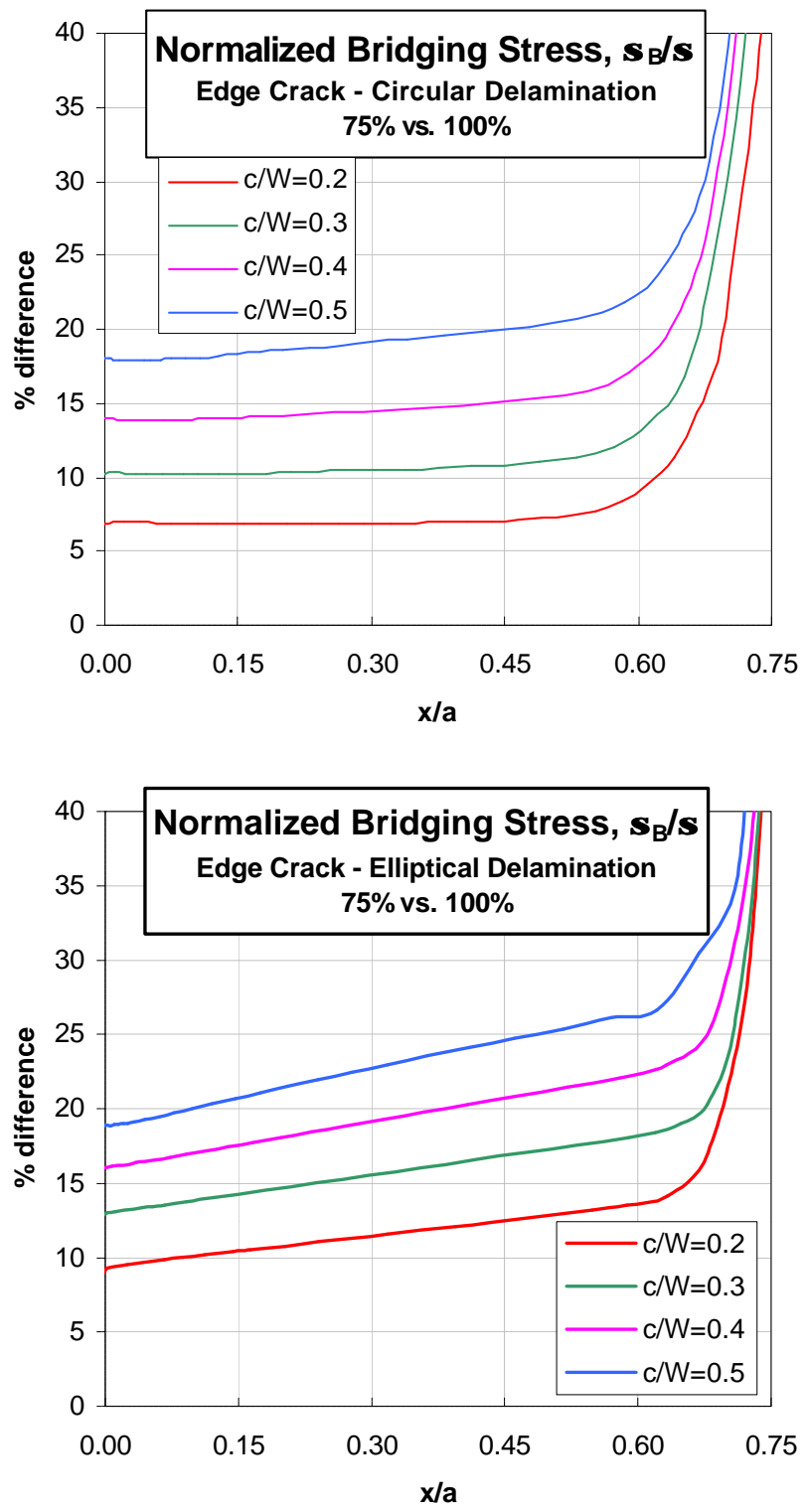

Figure 5.9: Percentage difference between $75 \%$ and $100 \%$ bridging, for the variations of the normalized bridging stress, $\sigma_{\mathrm{B}} / \sigma$, with relative distance from the crack-tip, $x / a$, along the centreline of the aramidepoxy layer. Edge crack problem with circular and elliptical delaminations. 

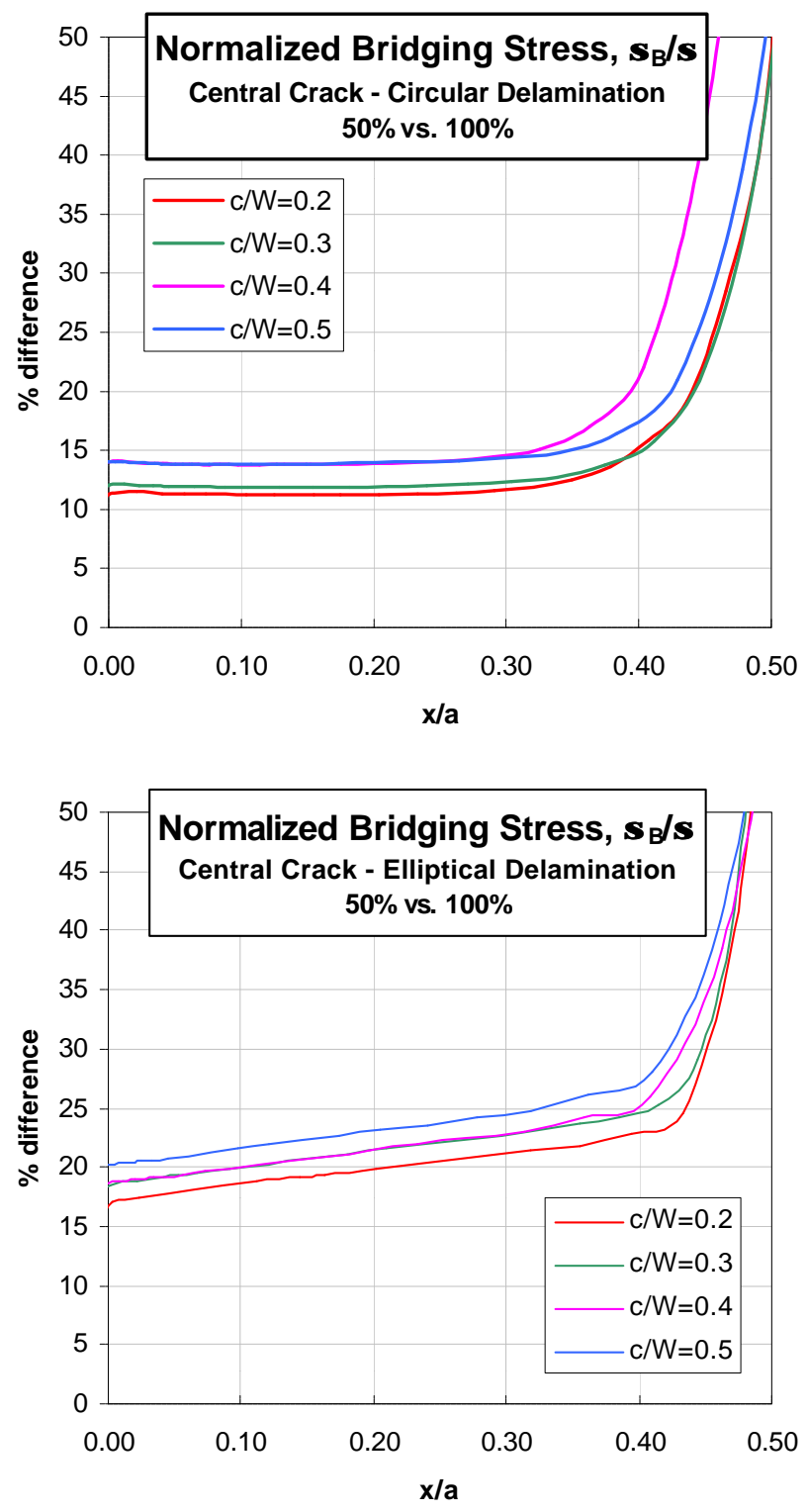

Figure 5.10: Percentage difference between 50\% and $100 \%$ bridging, for the variations of the normalized bridging stress, $\sigma_{\mathrm{B}} / \sigma$, with relative distance from the crack-tip, $x / a$, along the centreline of the aramidepoxy layer. Central crack problem with circular and elliptical delaminations. 

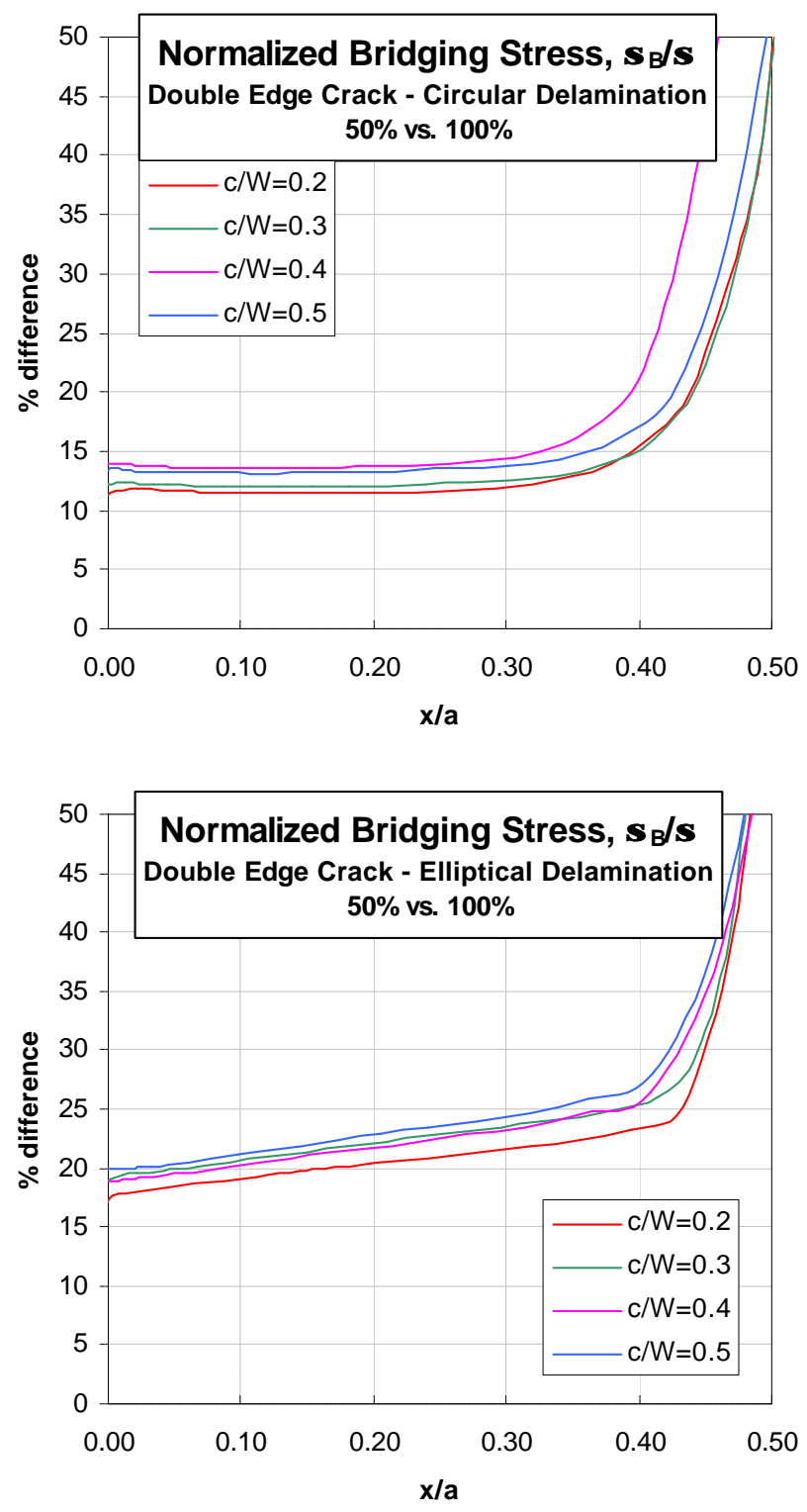

Figure 5.11: Percentage difference between $50 \%$ and $100 \%$ bridging, for the variations of the normalized bridging stress, $\sigma_{\mathrm{B}} / \sigma$, with relative distance from the crack-tip, $x / a$, along the centreline of the aramidepoxy layer. Double edge crack problem with circular and elliptical delaminations. 

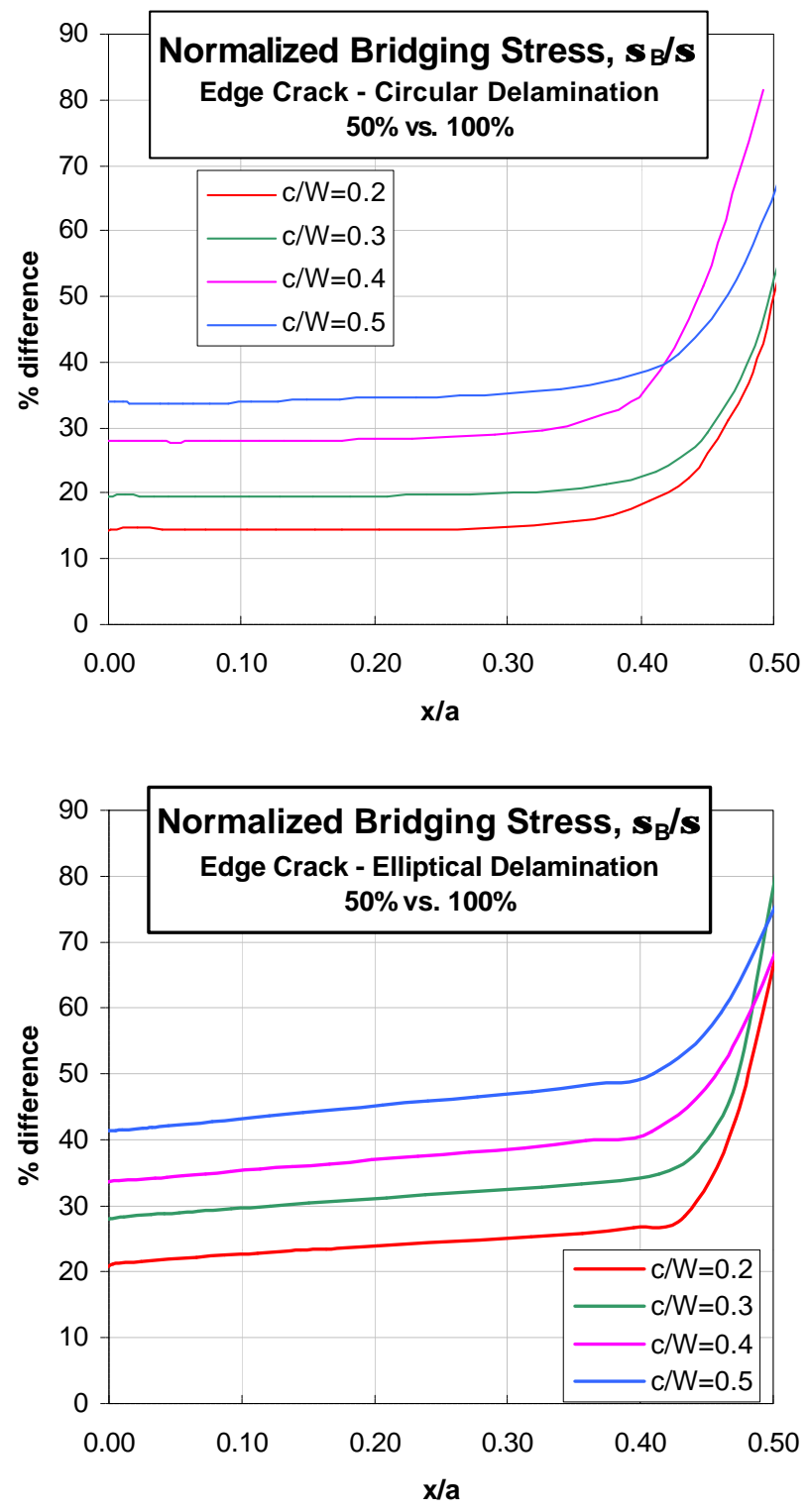

Figure 5.12: Percentage difference between $50 \%$ and $100 \%$ bridging, for the variations of the normalized bridging stress, $\sigma_{\mathrm{B}} / \sigma$, with relative distance from the crack-tip, $x / a$, along the centreline of the aramidepoxy layer. Edge crack problem with circular and elliptical delaminations. 
Table 5.1: Percentage difference of normalized stress intensity factors, $K_{I} / \sigma(\pi c)^{1 / 2}$, between partial bridging and full bridging obtained from BIE analysis for different relative crack lengths, $\mathrm{c} / \mathrm{W}$, and power-law indices: Central crack with circular and elliptical delamination.

\begin{tabular}{|c|c|c|c|c|c|c|c|}
\hline & \multicolumn{3}{|c|}{ Elliptical Delamination } & \multicolumn{3}{|c|}{ Circular Delamination } \\
\hline & & \multirow{2}{*}{$\begin{array}{c}75 \% \\
\% \text { diff }\end{array}$} & \multirow{2}{*}{$\begin{array}{c}50 \% \\
\% \text { diff }\end{array}$} & \multirow{2}{*}{$\begin{array}{c}0 \% \\
\% \text { diff }\end{array}$} & \multirow{2}{*}{$\begin{array}{c}75 \% \\
\% \text { diff }\end{array}$} & \multirow{2}{*}{$\begin{array}{c}50 \% \\
\% \text { diff }\end{array}$} & \multirow{2}{*}{$\begin{array}{c}0 \% \\
\% \text { diff }\end{array}$} \\
\hline Index & c/W & & & & & & \\
\hline \multirow[t]{4}{*}{$\mathrm{n}=\mathrm{m}=\mathbf{0}$} & 0.2 & 11.4 & 21.0 & 43.5 & 3.6 & 7.9 & 32.8 \\
\hline & 0.3 & 13.1 & 23.8 & 47.9 & 2.9 & 7.3 & 34.9 \\
\hline & 0.4 & 14.8 & 26.3 & 50.1 & 3.5 & 8.3 & 36.2 \\
\hline & 0.5 & 15.9 & 28.1 & 51.8 & 3.7 & 9.2 & 37.3 \\
\hline \multirow[t]{4}{*}{$\mathrm{n}=0.5$} & 0.2 & 5.2 & 13.2 & 38.4 & -1.5 & -2.0 & 24.6 \\
\hline & 0.3 & 5.5 & 13.0 & 38.3 & -1.7 & -2.4 & 24.5 \\
\hline & 0.4 & 6.0 & 13.2 & 38.5 & -1.2 & -2.0 & 24.8 \\
\hline & 0.5 & 6.0 & 13.6 & 39.6 & -1.4 & -1.9 & 25.7 \\
\hline \multirow[t]{4}{*}{$\mathbf{n}=1.0$} & 0.2 & 3.1 & 8.7 & 27.8 & -1.3 & -1.5 & 17.5 \\
\hline & 0.3 & 3.3 & 8.8 & 28.1 & -1.5 & -1.9 & 17.5 \\
\hline & 0.4 & 3.9 & 9.3 & 28.7 & -1.1 & -1.5 & 18.0 \\
\hline & 0.5 & 4.2 & 9.9 & 30.0 & -1.0 & -1.3 & 19.0 \\
\hline
\end{tabular}

Table 5.2: Percentage difference of normalized stress intensity factors, $K_{I} / \sigma(\pi c)^{1 / 2}$, between partial bridging and full bridging obtained from BIE analysis for different relative crack lengths, $\mathrm{c} / \mathrm{W}$, and power-law indices: Double edge crack with circular and elliptical delamination.

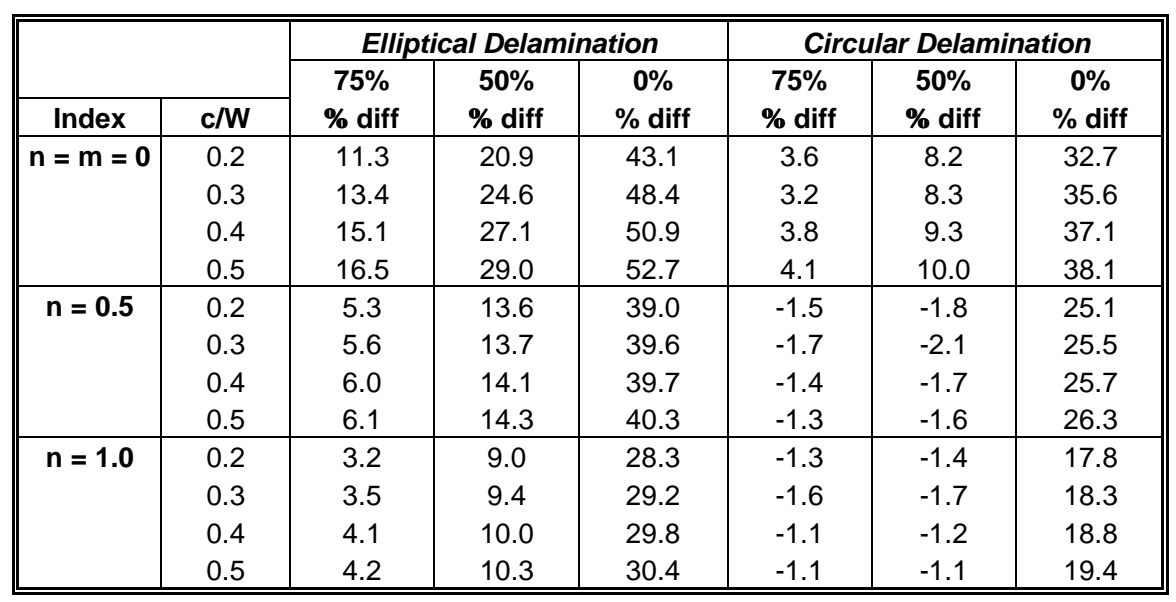


Table 5.3: Percentage difference of normalized stress intensity factors, $K_{I} / \sigma(\pi c)^{1 / 2}$, between partial bridging and full bridging obtained from BIE analysis for different relative crack lengths, $\mathrm{c} / \mathbf{2 W}$, and power-law indices: Edge crack with circular and elliptical delamination.

\begin{tabular}{|c|c|c|c|c|c|c|c|}
\hline & \multicolumn{3}{|c|}{ Elliptical Delamination } & \multicolumn{3}{|c|}{ Circular Delamination } \\
\hline & & \multirow{2}{*}{$\begin{array}{c}75 \% \\
\% \text { diff }\end{array}$} & \multirow{2}{*}{$\begin{array}{c}50 \% \\
\% \text { diff }\end{array}$} & \multirow{2}{*}{$\begin{array}{c}0 \% \\
\% \text { diff }\end{array}$} & \multirow{2}{*}{$\begin{array}{l}75 \% \\
\% \text { diff }\end{array}$} & \multirow{2}{*}{$\begin{array}{c}50 \% \\
\% \text { diff }\end{array}$} & \multirow{2}{*}{$\begin{array}{c}0 \% \\
\% \text { diff }\end{array}$} \\
\hline Index & $\mathrm{c} / 2 \mathrm{~W}$ & & & & & & \\
\hline \multirow[t]{4}{*}{$\mathbf{n}=\mathbf{m}=\mathbf{0}$} & 0.2 & 11.9 & 21.6 & 42.3 & 5.1 & 10.9 & 33.2 \\
\hline & 0.3 & 17.0 & 29.8 & 50.5 & 7.9 & 16.5 & 40.0 \\
\hline & 0.4 & 23.7 & 38.5 & 57.2 & 13.6 & 25.3 & 47.1 \\
\hline & 0.5 & 31.0 & 47.2 & 63.4 & 20.9 & 35.6 & 54.8 \\
\hline \multirow[t]{4}{*}{$\mathrm{n}=0.5$} & 0.2 & 7.1 & 17.2 & 43.5 & -0.7 & 0.2 & 28.8 \\
\hline & 0.3 & 10.4 & 23.1 & 51.1 & 0.4 & 3.4 & 35.3 \\
\hline & 0.4 & 14.6 & 30.8 & 59.9 & 3.0 & 9.0 & 44.1 \\
\hline & 0.5 & 18.3 & 38.9 & 69.6 & 5.8 & 16.1 & 55.1 \\
\hline \multirow[t]{4}{*}{$n=1.0$} & 0.2 & 4.8 & 12.4 & 32.9 & -0.7 & 0.2 & 21.2 \\
\hline & 0.3 & 7.8 & 18.1 & 40.9 & 0.1 & 2.8 & 27.3 \\
\hline & 0.4 & 11.8 & 25.6 & 50.6 & 2.3 & 7.8 & 36.0 \\
\hline & 0.5 & 15.9 & 34.3 & 61.6 & 5.1 & 14.6 & 47.3 \\
\hline
\end{tabular}

\subsubsection{2-D BEM Analysis}

\section{Bridging Stress Distribution}

The normalized bridging stress, $\sigma_{\mathrm{B}}{ }^{*} / \sigma$, distribution for ARALL as a function of normalized crack length, $c / W$ or $c / 2 W$, is plotted in Figures 5.13 to 5.15 for each of the cases treated. These figures show the variations of $\sigma_{\mathrm{B}}{ }^{*} / \sigma$ for $75 \%$ and $50 \%$ partial bridging cases with varying fibre-bridging power-law indices, $n$ and $m$, and circular and elliptical delamination zone shapes.

The variations of normalized bridging stress, $\sigma_{\mathrm{B}}{ }^{*} / \sigma$, with crack length, $c / W$ (or $c / 2 W)$ are the same for all the partial bridging cases as they were for the full bridging case discussed in Section 4. However, the magnitudes of the bridging stresses increase as the bridging length, $b$, is decreased. The percentage differences of the stress between the $75 \%$ and 50\% partial bridging cases and that for full bridging are tabulated in Tables 5.7 to 5.9 , for the central, double edge, and edge cracks. The trend in percentage difference of the normalized bridging stress for the partial bridging cases compared to full bridging is shown graphically in Figure 5.16 for the central, double edge, and edge crack problems with an elliptical delamination and power-law index, $\mathrm{n}=0.5$.

The two-dimensional BIE results also show an increase of the normalized bridging stress, $\sigma_{\mathrm{B}}{ }^{*} / \sigma$, with a decreasing amount of fibres bridging the crack. The 
largest percent difference of $49.4 \%$ is for the edge crack with $50 \%$ bridging, circular delamination, $c / 2 W=0.5$, and power-law index $m=0.5$.
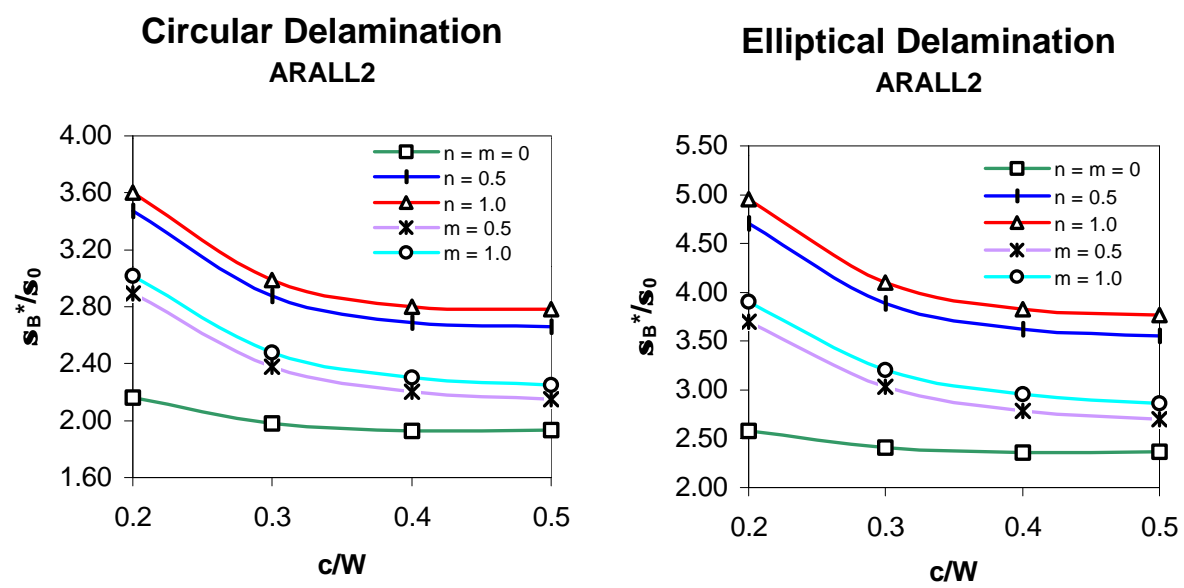

(a)

Circular Delamination

ARALL2

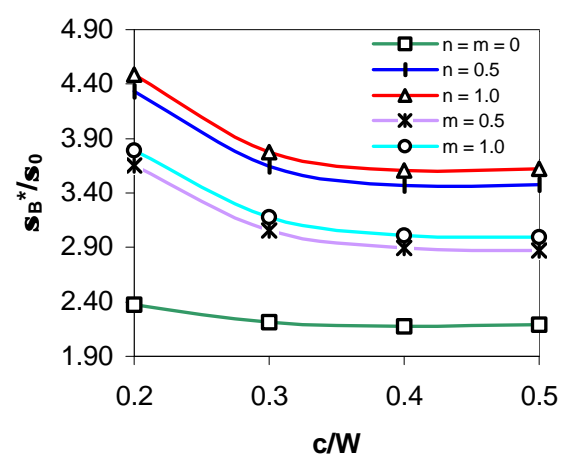

Elliptical Delamination

ARALL2

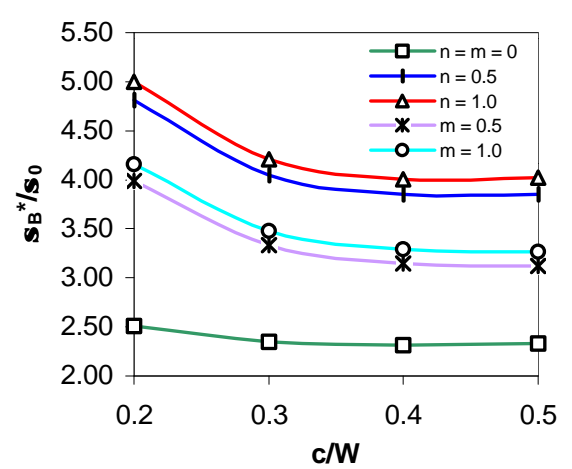

(b)

Figure 5.13: Variations of normalized bridging stress, $\sigma_{\mathrm{B}}{ }^{*} / \sigma$, with relative crack length, $\mathrm{c} / \mathrm{W}$, for the ARALL central crack problem containing circular and elliptical delamination with (a) $75 \%$ partial bridging, and (b) $50 \%$ partial bridging. 

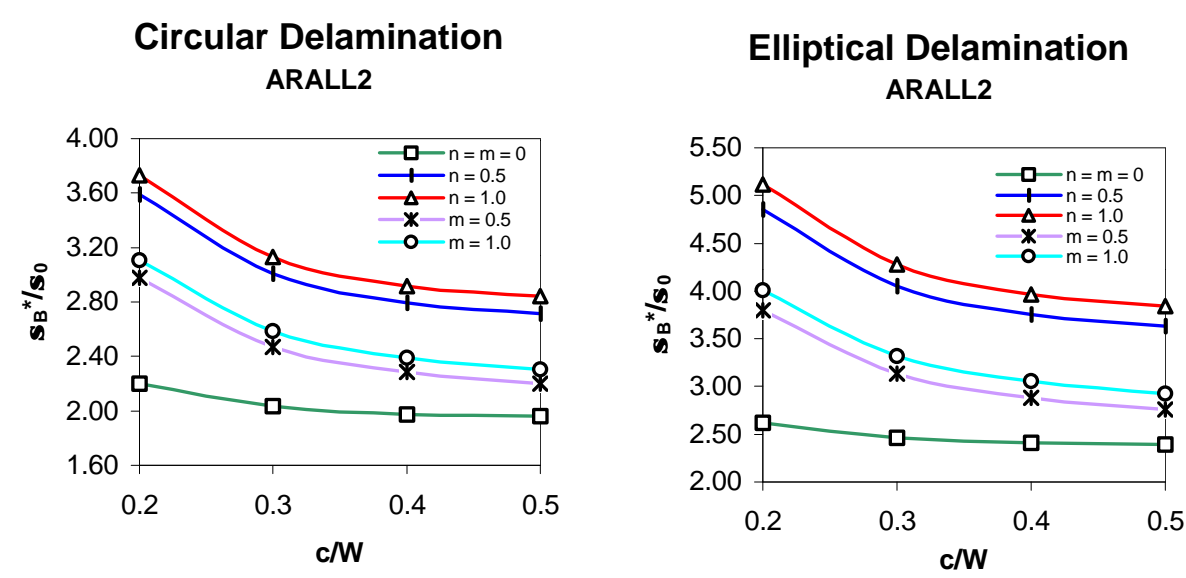

(a)
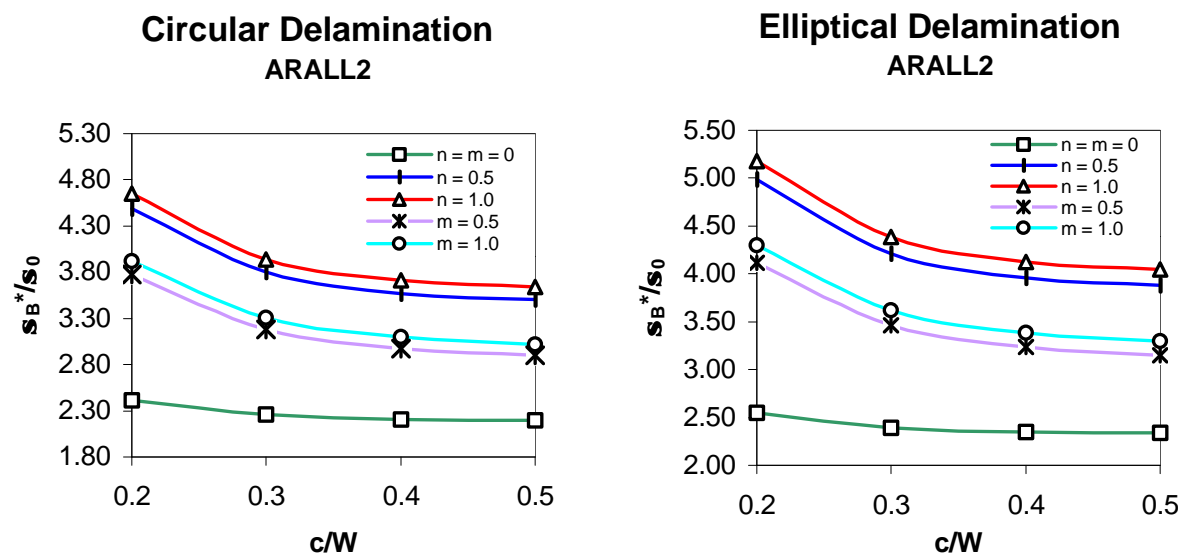

(b)

Figure 5.14: Variations of normalized bridging stress, $\sigma_{\mathrm{B}}{ }^{*} / \sigma$, with relative crack length, $\mathrm{c} / \mathrm{W}$, for the ARALL double edge crack problem containing circular and elliptical delamination with (a) $75 \%$ partial bridging, and (b) $50 \%$ partial bridging. 
Circular Delamination

ARALL2

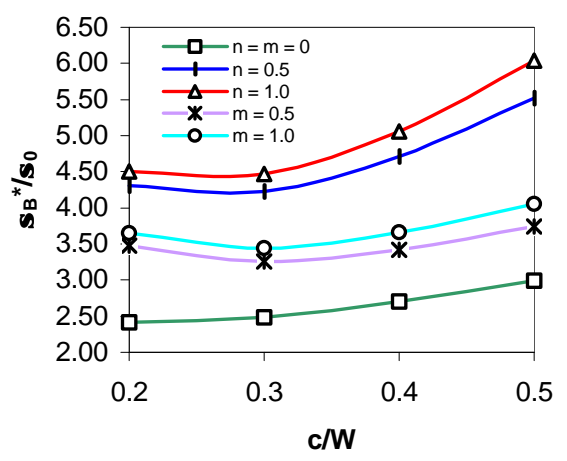

Elliptical Delamination ARALL2

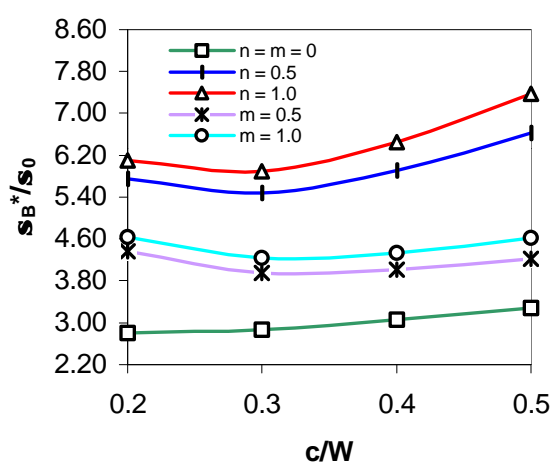

(a)

\section{Circular Delamination ARALL2}

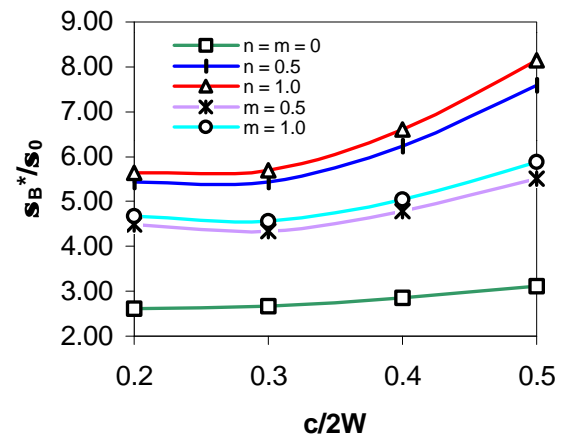

Elliptical Delamination ARALL2

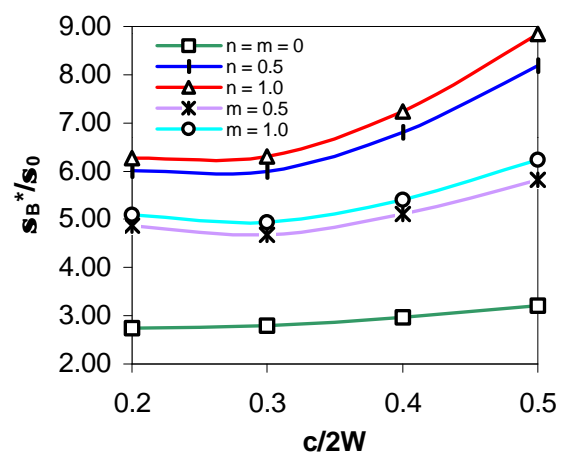

(b)

Figure 5.15: Variations of normalized bridging stress, $\sigma_{\mathrm{B}}{ }^{*} / \sigma$, with relative crack length, c/2W, for the ARALL edge crack problem containing circular and elliptical delamination with (a) $75 \%$ partial bridging, and (b) $50 \%$ partial bridging. 


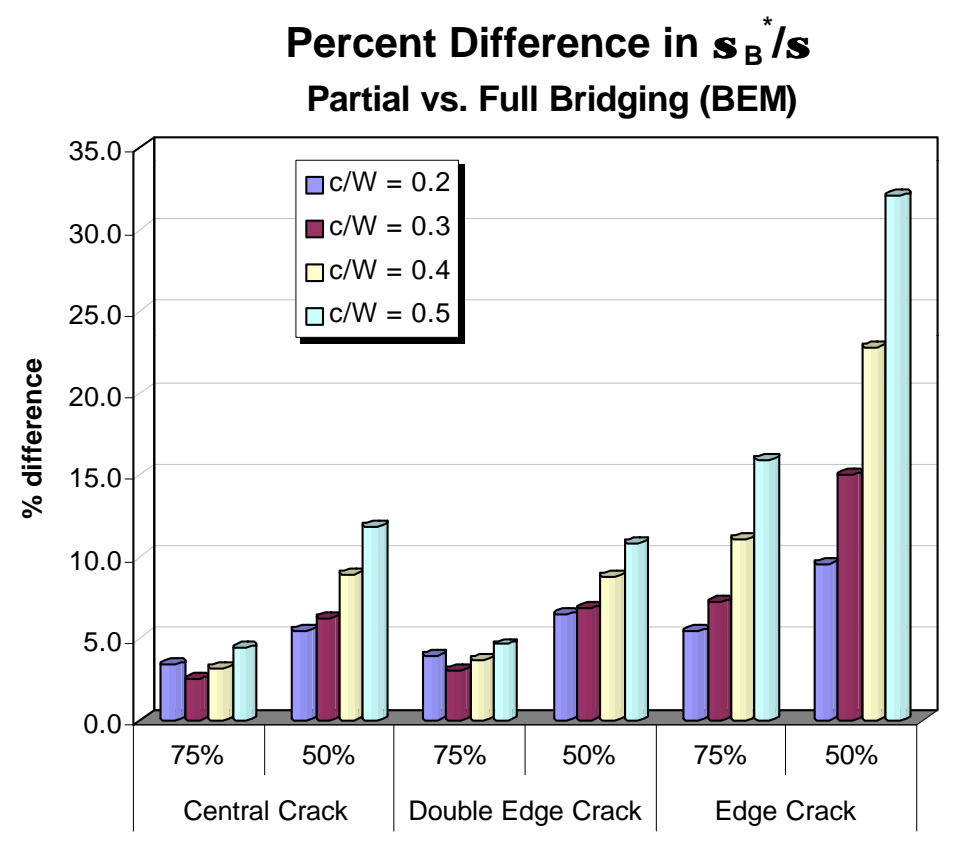

Figure 5.16: Percentage difference between the partial bridging cases and full bridging of the normalized bridging stress, $\sigma_{\mathrm{B}} * / \sigma$, for the central, double edge, and edge crack problem. Power-law index, $\mathrm{n}=0.5$ and an elliptical delamination. (BEM Analysis)

\section{Stress Intensity Factors}

The normalized crack-tip stress intensity factor, $K_{I} / \sigma \sqrt{\pi c}$, obtained from the BIE analysis are plotted with respect to the normalized crack length, $c / W$ or $c / 2 W$, in Figures 5.17 to 5.19, for the central, double edge, and edge cracks, respectively. In these figures, the normalized crack-tip stress intensity factor results from the threedimensional FEM analysis are also included for comparison.

The normalized stress intensity factor, $K_{I} / \sigma \sqrt{\pi c}$, generally increases with increasing power-law index $n$ and $m$, and crack length, $c / W$ or $c / 2 W$, for the cracked geometries considered with the partial bridging. The delamination zone shape also influences the magnitude of the normalized stress intensity factor; a smaller delamination size results in lower crack-tip stress intensity factors. This can be seen in 
Tables 5.1 to 5.3 and Figures 5.17 to 5.19 , that the normalized stress intensity factors are lower for the elliptical delamination than for the circular delamination for a given crack size.

It can also be seen for these results that as the length of the bridging zone, $b$, decreases, the normalized stress intensity factors generally increase. This is to be expected, for the crack is able to open up more when the amount of bridging fibres decreases. Comparing, for example, a central crack with $n=0.5$, elliptical delamination and $c / W=0.5, K_{I} / \sigma \sqrt{\pi c}=0.730$ for $100 \%$ bridging, $K_{I} / \sigma \sqrt{\pi c}=0.777$ for $75 \%$ bridging and $K_{I} / \sigma \sqrt{\pi c}=0.845$ for $50 \%$ bridging. Compared to $100 \%$ bridging, this corresponds to an increase of $6.0 \%$ and $13.6 \%$ for the $75 \%$ and $50 \%$ partial bridging cases, respectively. Figure 5.20 shows the percentage difference of the normalized stress intensity factor, $K_{I} / \sigma \sqrt{\pi c}$, between the partial bridging cases and full bridging for the central, double edge, and edge crack problems with an elliptical delamination. This figure also shows the trends of the normalized stress intensity factors for $75 \%$ and $50 \%$ bridging between the results obtained from the BIE and FEM analyses.

The normalized stress intensity factors, $K_{I} / \sigma \sqrt{\pi c}$, for an unbridged ARALL central crack, double edge crack, and edge crack problem are shown in Figure 5.21. For the previous example of the central crack, with $n=0.5$, elliptical delamination and $c / W$ $=0.5$, the normalized stress intensity factor for the unbridged case is 1.21 , which is $39.6 \%$ higher than the corresponding value for full bridging. Figure 5.21 also shows the normalized stress intensity factors from the FEM analysis calculated in the aluminum layer and the fibre-epoxy layer. It is expected that the stress intensity factors in the fibre layer should be less than those in the aluminum layer since the restraint of fibres on crack opening is greater than for aluminum. This is confirmed in these figures where the crack-tip normalized stress intensity factors are much lower in the fibre layer than the aluminum layer.

For the stress intensity factors calculated in the aluminum layer, the BEM results are approximately $10 \%$ lower than the FEM results. For the stress intensity factors calculated in the fibre-epoxy layer, the BEM results are approximately 25 to $30 \%$ higher than the FEM results. Therefore, the two-dimensional BEM analysis predicts the crack-tip stress intensity factors in the aluminum layer to within $10 \%$ of the actual three-dimensional result. 

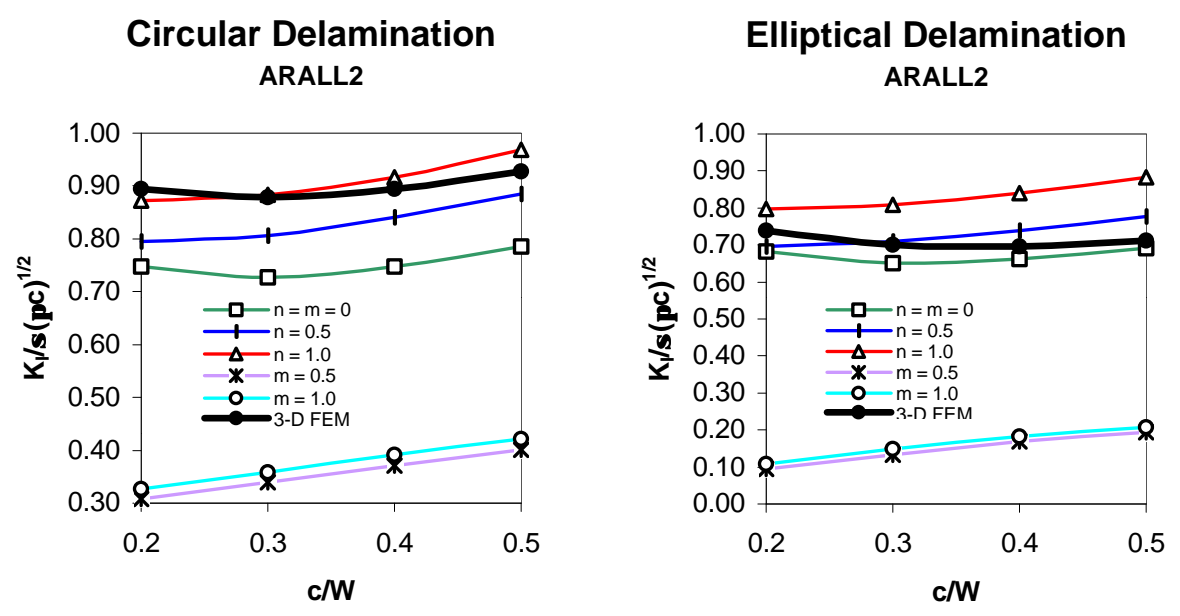

(a)

\section{Circular Delamination}

ARALL2

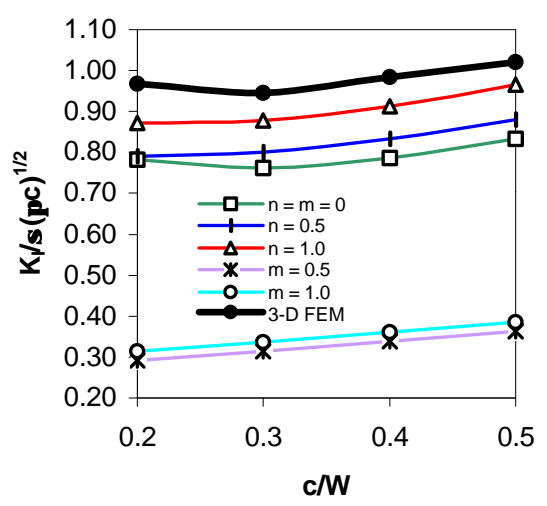

Elliptical Delamination ARALL2

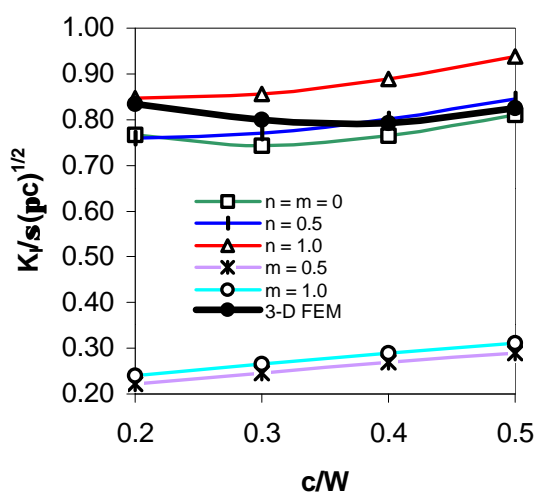

(b)

Figure 5.17: Variations of normalized crack-tip stress intensity factor, $K_{\mathrm{I}} / \sigma(\pi \mathrm{c})^{1 / 2}$, with relative crack length, $\mathrm{c} / \mathrm{W}$, for the ARALL central crack problem containing circular and elliptical delamination with (a) 75\% partial bridging, and (b) $50 \%$ partial bridging. 

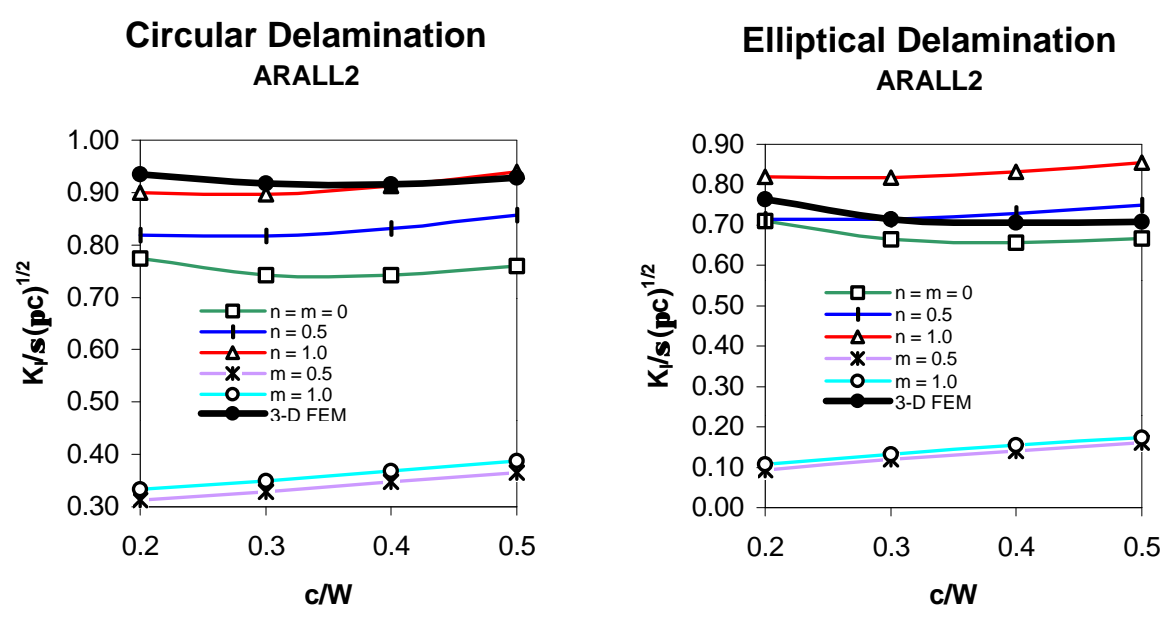

(a)
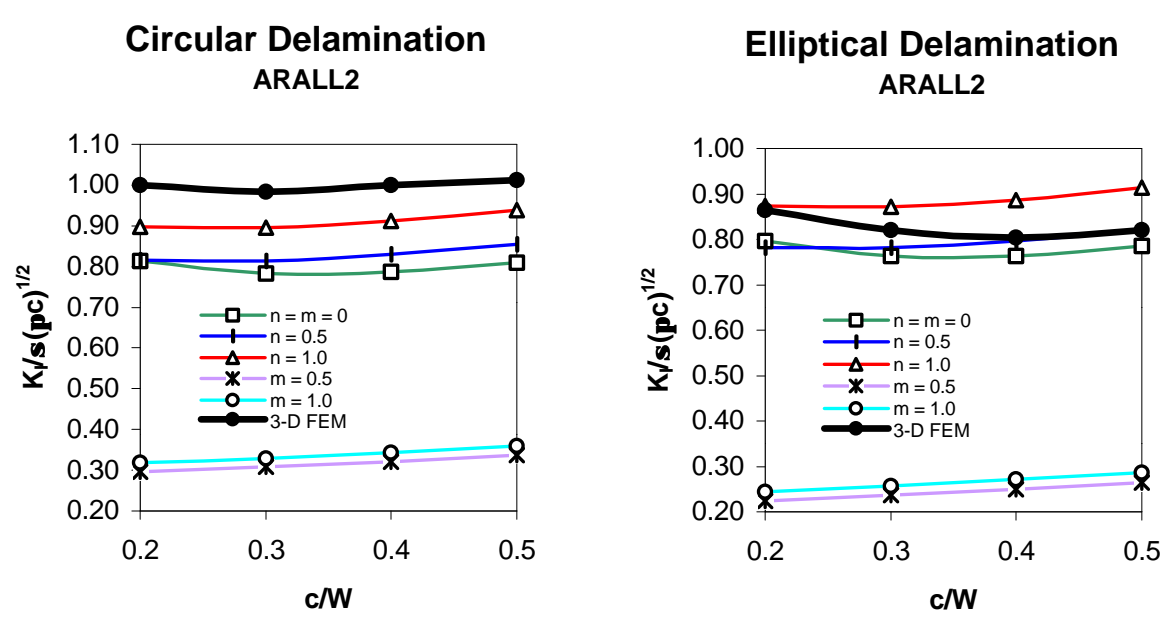

(b)

Figure 5.18: Variations of normalized crack-tip stress intensity factor, $K_{I} / \sigma(\pi c)^{1 / 2}$, with relative crack length, $\mathrm{c} / \mathrm{W}$, for the ARALL double edge crack problem containing circular and elliptical delamination with (a) 75\% partial bridging, and (b) $50 \%$ partial bridging. 


\section{Circular Delamination ARALL2}

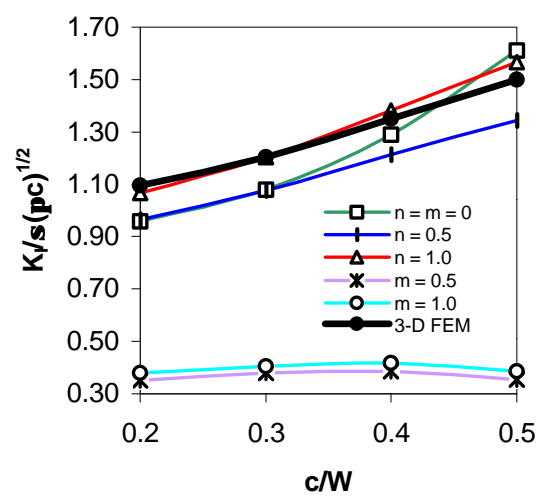

\section{Elliptical Delamination} ARALL2

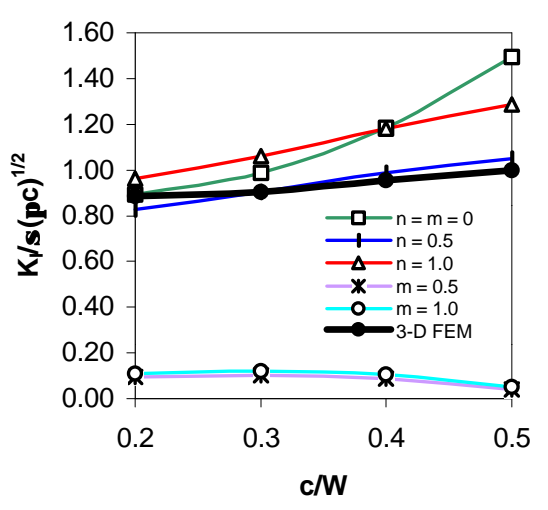

(a)
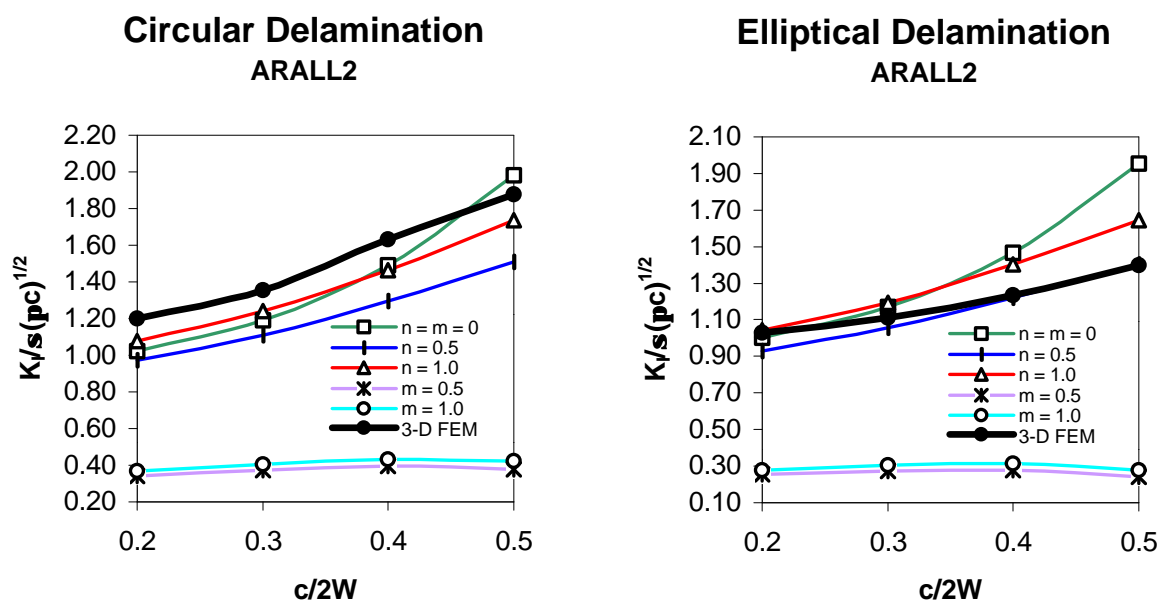

(b)

Figure 5.19: Variations of normalized crack-tip stress intensity factor, $K_{I} / \sigma(\pi c)^{1 / 2}$, with relative crack length, $\mathrm{c} / \mathbf{2} \mathrm{W}$, for the ARALL edge crack problem containing circular and elliptical delamination with (a) 75\% partial bridging, and (b) $50 \%$ partial bridging. 


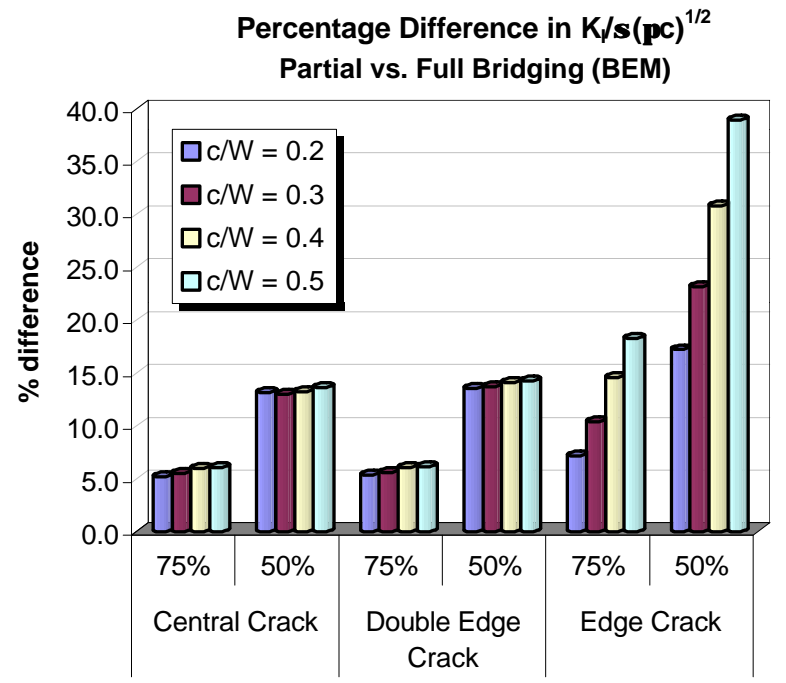

(a)

Percent Difference in $\mathrm{K}_{\mathrm{l}} / \sigma(\pi \mathrm{c})^{1 / 2}$

Partial vs. Full Bridging (FEM)

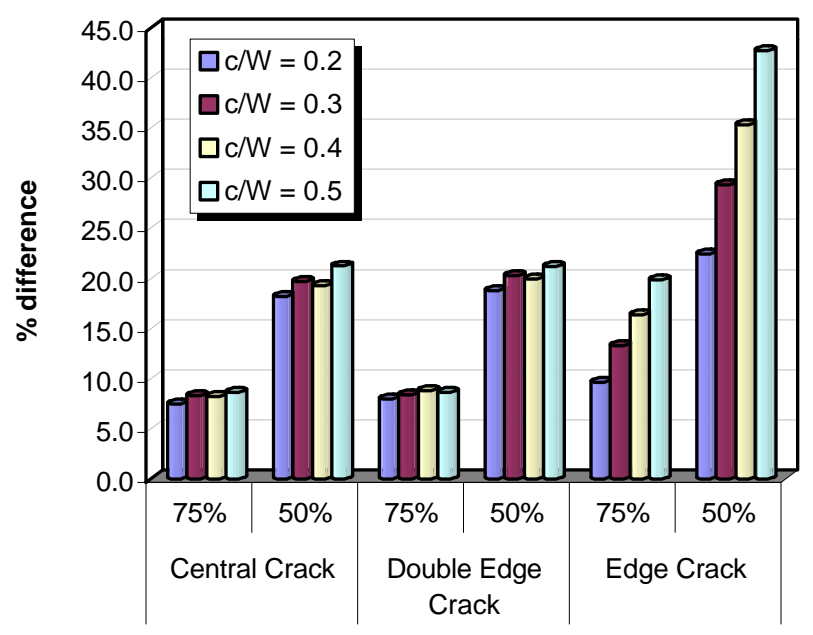

(b)

Figure 5.20: Percentage difference between the partial bridging cases and full bridging of the normalized stress intensity factor, $K_{\mathrm{I}} / \sigma(\pi \mathrm{c})^{1 / 2}$, for the central, double edge, and edge crack problem with an elliptical delamination; (a) BEM analysis, and (b) FEM analysis. 


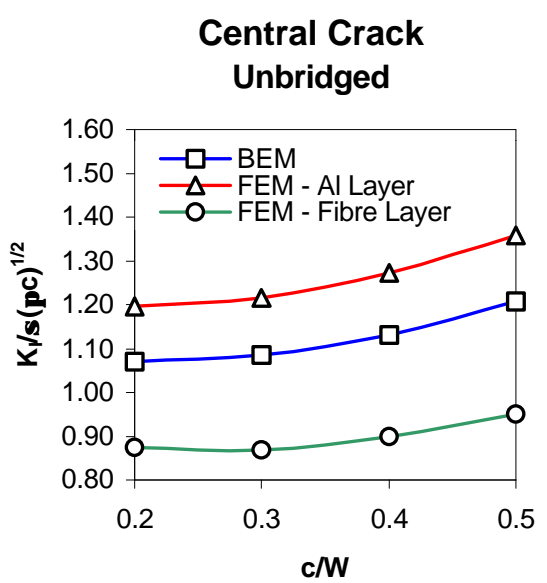

(a)
Double Edge Crack Unbridged

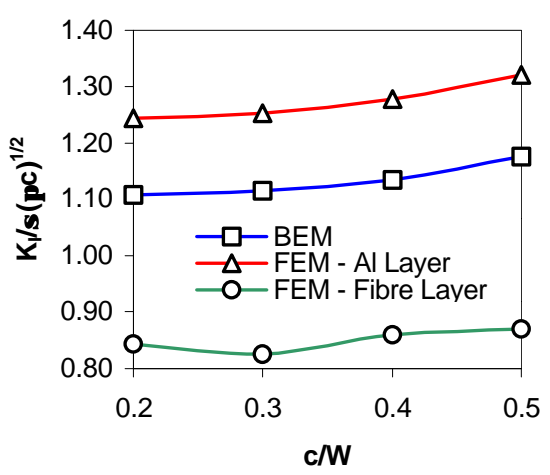

(b)

\section{Edge Crack}

Unbridged

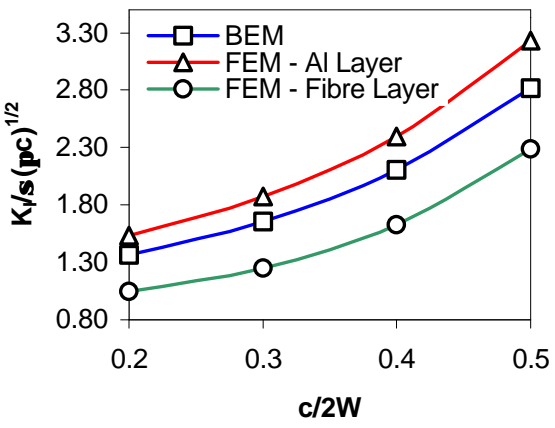

(c)

Figure 5.21: Variations of the normalized stress intensity factor, $K_{\mathrm{I}} / \sigma(\pi \mathrm{c})^{1 / 2}$, with relative crack length, $\mathrm{c} / \mathrm{W}$ or $\mathrm{c} / \mathbf{2 W}$, for the ARALL (a) central crack problem, (b) double edge crack problem, and (c) edge crack problem. Results from the BEM and the FEM for an unbridged fibre-metal laminate. 


\subsection{Conclusions}

The bridging mechanism in fibre-metal laminates has been described and because of its simplicity, the stress intensity approach with power-law expressions for bridging stresses has been chosen to analyse fibre-metal laminates. The three dimensional bridging mechanism in fibre-metal laminates has been reduced to a two-dimensional one with the assumption of homogenised material properties through the thickness, and the bridging stresses as a closing pressure on the crack flanks. Since these bridging stresses are initially unknown, this two-dimensional, mathematically non-linear problem has to be solved iteratively and a numerical technique to solve for these unknown bridging stresses has been presented.

The two-dimensional analysis was carried out using the boundary element method (BEM) and a review of this method for anisotropic linear elasticity has been presented along with some of the principles of linear-elastic fracture mechanics (LEFM). A threedimensional analysis of three rectangular fibre-metal laminate plates with cracks emanating from the edge of a circular cut-out and under remote uniform tension in the fibre direction, was performed using FEM. The applicability of a two-dimensional analysis using BEM was presented along with the discussion of the influence of varying crack size, geometry, bridging stress profile and delamination zone size on the bridging stress and stress intensity factors. A comparison was then made between the results obtained from the 2-D BEM analysis and the 3-D FEM analysis.

The results of the BEM and FEM analysis for the case of full bridging can be summarized as follows:

- A high normalized bridging stress, $\sigma_{\mathrm{B}} / \sigma$, at the crack mouth, was obtained indicating high strain in the fibres there and therefore the likelihood of fibre failure near the edge of the hole.

- The high normalized bridging stress is generally confined to a $5 \%$ to $10 \%$ region of the total crack length.

- $\quad$ The normalized stress intensity factors also increase with increasing power-law index, $n$ and $m$, but the power-law index $m$ does not accurately predict stress intensity factors and hence cannot be applied to the analysis of notched fibremetal laminates with cracks.

- The FEM results lie between $0 \leq n \leq 1.0$ of the BEM results, but are generally closer to $n=0.5$, which describes a decreasing $\sigma^{*}{ }_{\mathrm{B}} / \sigma$ towards crack tip indicating the behaviour of a 'strain-hardening' material.

The effect of the extent of fibre-bridging on the bridging mechanism has been examined in this study. This investigation considered $75 \%$ and $50 \%$ of the crack being bridged by fibres and the results from the FEM and BEM analysis were presented. The conclusions from the partial bridging analysis are itemized below.

- The normalized bridging stress, $\sigma_{\mathrm{B}} / \sigma$ increases with a decreasing bridging length, $b$, but the normalized stress intensity factors, $\mathrm{K}_{\mathrm{I}} / \sigma(\pi \mathrm{c})^{1 / 2}$, increase with a decreasing bridging length due to more opening of the crack faces as a result of less fibres bridging the crack. 
- The normalized bridging stress, $\sigma_{\mathrm{B}} / \sigma$, for $75 \%$ and $50 \%$ bridging was found to be $5 \%$ to $13 \%$ and $11 \%$ to $26 \%$ higher than full bridging, respectively, for the central and double edge crack problems. For the edge crack problem, the corresponding differences are about $7 \%$ to $25 \%$ and $15 \%$ to $50 \%$ higher than full bridging case.

The benefit of fibre-bridging in fibre-metal laminates was quantified with the FEM and BEM analysis of an unbridged crack. The stress intensity factors were found to be much higher when there is no bridging of the crack as compared to full bridging, and even some partial bridging. The study has clearly demonstrated that the simplified 2-D BEM analysis can provide reasonable estimates of the stress intensity factor for cracks in fibre-metal laminates with stress concentrations. It was less time consuming and much easier to obtain the estimates than a corresponding 3-D finite element analysis.

\section{References}

Ang, H.E., Y.L. Gao and C.L. Tan, (1992). "Strength Prediction of Unidirectional Composites with a Circular Hole using Fracture Mechanics". International Journal of Fracture. 56, pp. R23-R29.

Cain, K.J. and C.L. Tan, (1997). "Numerical Modelling of Fiber-Bridging in Cracked Fiber Metal Laminate". Journal of Advanced Materials. 28, pp. 8-15.

Chen, J.L. and C.T. Sun, (1989). "Modeling of Orthotropic Elastic-Plastic Properties of ARALL Laminates". Composite Science and Technology. 36, pp. 321-337.

El-Zein, M.S. and K.L. Reifsnider, (1990). "The Strength Prediction of Composite Laminates Containing a Circular Hole". Journal of Composites Technology and Research. 12, pp. 24-30.

Foote, R.M.L., Y. Mai and B. Cotterell, (1986). "Crack Growth Resistance Curves in Strain-Softening Materials", Journal of the Mechanics and Physics of Solids. 34, pp. 593-607.

Gibson, R.F., (1994). Principles of Composite Material Mechanics. McGraw-Hill, Inc., Toronto.

Guinea, G.V., J. Planas and M. Elices, (2000). " $\mathrm{K}_{\mathrm{I}}$ Evaluation by the Displacement Extrapolation Technique". Engineering Fracture Mechanics. 66, pp. 243-255. 
Guo, Y.J. and X.R. Wu, (1998). "A Theoretical Model for Predicting Fatigue Crack Growth Rates in Fibre-Reinforced Metal Laminates". Fatigue \& Fracture of Engineering Materials \& Structures. 21, pp. 1133-1145.

Guo, Y-J. and X-R. Wu, (1999). "Bridging Stress Distribution in Center-Cracked Fiber Reinforced Metal Laminates: Modeling and Experiment". Engineering Fracture Mechanics. 63, pp. 147-163.

Lin, C.T. and P.W. Kao, (1996). "Delamination Growth and Its Effect on Crack Propagation in Carbon Fiber Reinforced Aluminum Laminates Under Fatigue Loading”. Acta. Mater. 44, pp. 1181-1188.

Macheret, J., J.L. Teply and E.F.M. Winter, (1988). "Delamination Shape Effects in ARALL $®$ Laminates with Fatigue Cracks". Presented at the $8^{\text {th }}$ annual NRCC/IMRI symposium “Composites- '88'”, Nov 8-9, Boucherville, Que., Canada.

Marissen, R., (1988). "Fatigue Crack Growth Predictions in Aramid Reinforced Aluminum Laminates (ARALL)", Journal of Aircraft. 25, pp. 135-40.

Marissen, R., (1989). "Mechanical Aspects Related to Fibre Fracture in ARALL Laminates", Advances in Fatigue Science and Technology. C.M. Branco and L.G. Rosa (eds.), Kluwer Academic Publishers, pp. 697-707.

Marissen, R., (1991). "Interaction of Fatigue Mechanisms During Crack Growth in ARALL". IUTAM Symposium. J.N. Reddy and K.L. Reifsnider (eds.), Blacksburg, $V A$.

Marissen, R., (1994). "Flight Simulation Behaviour of Aramid Reinforced Aluminum Laminates (ARALL)”. Engineering Fracture Mechanics. 19, pp. 261-277.

Martinez, J. and J. Dominguez, (1984). "On the Use of Quarter-Point Boundary Elements for Stress Intensity Factor Computations", International Journal of Numerical Methods in Engineering. 20, pp. 1941-1950.

Ritche, R.O., Y. Weikang, and J. Bucci, (1989). "Fatigue Crack Propagation in ARALL Laminates: Measurement of the Effect of Crack-tip Shielding from Crack Bridging", Engineering Fracture Mechanics. 32, pp. 361-77.

Roebroeks, G.H.J.J., (1994). "Fibre-Metal Laminates: Recent Developments and Applications", Fatigue. 16, pp. 33-42.

Rushau, J.J., (1988). "Fatigue Crack Growth Characteristics of ARALL", University of Dayton Research Institute, AFWAL-TR-88-4075.

Schijve, J., (1994). "Fatigue of Aircraft Materials and Structures". Fatigue. 16, pp. 2132. 
B.E. Cudzilo and C. L. Tan / Electronic Journal of Boundary Elements, Vol. 1, No. 3, pp. 336-403 (2003)

Schwartz, M.M., (1997). Composite Materials, Vol. 1. Prentice Hall, Upper Saddle River.

Suzuki, T. and M. Sakai, (1994). “A Model for Crack-face Bridging”, International Journal of Fracture. 65, pp. 329-44.

Tada, H., P.C. Paris and G.R. Irwin, (1973). The Stress Analysis of Cracks Handbook. Del Research Corporation, Pennsylvania, USA.

Takamatsu, T., T. Matsumura, N. Ogura, T. Shimokawa, and Y. Kakuta, (1999). "Fatigue Crack Growth Properties of a GLARE3-5/4 fiber/metal laminate". Engineering Fracture Mechanics. 63, pp. 253-272.

Tan, C.L., (1987). The Boundary Element Method: A Short Course. Carleton University, Ottawa, Canada.

Tan, C.L. and Y.L. Gao, (1992a). "Boundary Integral Equation Fracture Mechanics Analysis of Plane Orthotropic Bodies", International Journal of Fracture. 53, pp. $343-65$.

Tan, C.L. and Y.L. Gao, (1992b). "Boundary Element Analysis of Plane Anisotropic Bodies with Stress Concentrations and Cracks", Composite Structures. 20, pp. 1728.

Tan, C.L., Y.L. Gao and F.F. Afagh, (1992). “Anisotropic Stress Analysis of Inclusion Problems Using the Boundary Integral Equation Method". Journal of Strain Analysis. 27, pp. 67-76.

Yeh, J.R., (1988). "Fracture Mechanics of Delamination in ARALL Laminates", Engineering Fracture Mechanics. 30, pp. 827-37. 